\title{
Replication-Aware Linearizability
}

\author{
CONSTANTIN ENEA, University Paris Diderot, France \\ SUHA ORHUN MUTLUERGIL, University Paris Diderot, France \\ GUSTAVO PETRI, ARM Research, United Kingdom \\ CHAO WANG, University Paris Diderot, France
}

\begin{abstract}
Geo-distributed systems often replicate data at multiple locations to achieve availability and performance despite network partitions. These systems must accept updates at any replica and propagate these updates asynchronously to every other replica. Conflict-Free Replicated Data Types (CRDTs) provide a principled approach to the problem of ensuring that replicas are eventually consistent despite the asynchronous delivery of updates.

We address the problem of specifying and verifying CRDTs, introducing a new correctness criterion called Replication-Aware Linearizability. This criterion is inspired by linearizability, the de-facto correctness criterion for (shared-memory) concurrent data structures. We argue that this criterion is both simple to understand, and it fits most known implementations of CRDTs. We provide a proof methodology to show that a CRDT satisfies replication-aware linearizability which we apply on a wide range of implementations. Finally, we show that our criterion can be leveraged to reason modularly about the composition of CRDTs.
\end{abstract}

\section{INTRODUCTION}

Conflict-Free Replicated Data Types (CRDTs) [Shapiro et al. 2011] have recently been proposed to address the problem of availability of a distributed application under network partitions. CRDTs represent a methodological attempt to alleviate the problem of retaining some data-Consistency and Availability under network Partitions (CAP), famously known to be an impossible combination of requirements by the CAP theorem of Gilbert and Lynch [2002]. CRDTs are data types designed to favor availability over consistency by replicating the type instances across multiple nodes of a network, and allowing them to temporarily have different views. However, CRDTs guarantee that the different states of the nodes will eventually converge to a state common to all nodes [Burckhardt 2014; Shapiro et al. 2011]. This convergence property is intrinsic to the data type design and in general no synchronization is needed, hence achieving availability.

Availability vs. Consistency. To illustrate the problem we consider the implementation of a listlike CRDT object, the Replicated Growable Array (RGA) - due to Roh et al. [2011] ${ }^{1}$-, used for text-editing applications. RGA supports three operations: (1) addAfter $(a, b)$ which adds the character $\mathrm{b}$ - the concrete type is inconsequential here - immediately after the occurrence of the character a assumed to be present in the list, ${ }^{2}$ (2) remove (a) which removes a assumed to be present in the list, and (3) read () which returns the list contents.

To make the system available under partitions, RGA allows each of the nodes to have a copy of the list instance. We will call each of the nodes holding a copy a replica. Then the question is, how can we maintain the consistency of the different copies of the list given that the data could be at any point in time be modified or read by any of the replicas? A naive approach would synchronize all the

\footnotetext{
${ }^{1}$ We use a variation of code extracted from [Attiya et al. 2016].

${ }^{2} \mathrm{We}$ assume elements are unique, implemented with timestamps.
}

Authors' addresses: Constantin Enea, University Paris Diderot, France, cenea@irif.fr; Suha Orhun Mutluergil, University Paris Diderot, France, mutluergil@irif.fr; Gustavo Petri, ARM Research, United Kingdom, gustavo.petri@arm.com; Chao Wang, University Paris Diderot, France, wangch@irif.fr.

2018. 2475-1421/2018/1-ART1 \$15.00

https://doi.org/

Proc. ACM Program. Lang., Vol. 1, No. CONF, Article 1. Publication date: January 2018. 
replicas on each operation, hence maintaining coherence, but rendering the system unavailable if any one replica goes off-line.

Instead, RGA allows any of the replicas to modify the local copy of the list immediately - and hence return control to the client - and lazily propagate the updates to the other replicas. For instance, assuming that we have an initial list containing the sequence $a \cdot b \cdot e \cdot f^{3}$ and two replicas, $r_{1}$ and $r_{2}$, if $r_{1}$ inserts the letter $c$ after $b$ (calling addAfter $(b, c)$ ), while $r_{2}$ concurrently inserts the letter $d$ after $b($ addAfter $(b, d))$ the replicas will have the states $a \cdot b \cdot c \cdot e \cdot f$ and $a \cdot b \cdot d \cdot e \cdot f$ respectively. We have solved the availability problem, but we have introduced inconsistent states. This problem is only exacerbated by adding more replicas.

Convergence. To restore the replicas to a consistent state, CRDTs guarantee that under conflicting operations - that is, operations that could lead to different states - there is a systematic way to detect conflicts, and there is a strategy followed by all replicas to deterministically resolve conflicts.

In the case of RGA, the implementation adds metadata to each item of the list identifying the originating replica as well as timestamp of the operation in that replica. ${ }^{4}$ This metadata is enough to detect when conflicts have occurred. Generally there are a number of assumptions that are necessary for the metadata to detect conflicts (for instance that timestamps increase monotonically with time) which we shall discuss in the following sections. Then, for RGA it is enough to know whether two addAfter operations have conflicted by simply comparing the replica identifiers and their timestamps. In fact, this is a sound over-approximation of conflict since two concurrent addAfter operations have a real conflict only if their first arguments are the same (e.g. the element $b$ in the example aforementioned). In such case, the strategy to resolve the conflict will always choose to order first the character added with the highest timestamp in the resulting list, and in the particular case where the timestamps should be the same, an arbitrary order among replicas will be used. In the example above, and assuming that the character $c$ was added with timestamp $t_{1}$ and the character $\mathrm{d}$ was added with timestamp $t_{2}$, if $t_{2}<t_{1}$ (for some order $\leq$ between timestamps), the list will converge to $\mathrm{a} \cdot \mathrm{b} \cdot \mathrm{c} \cdot \mathrm{d} \cdot \mathrm{e} \cdot \mathrm{f}$. We obtain the same result if $t_{1}=t_{2}$ and assume that we have a replica order $<_{r}$, we have $r_{2}<_{r} r_{1}$. In any other case we obtain $a \cdot b \cdot d \cdot c \cdot e \cdot f$. Using an arbitrary order among replica identifiers is common in CRDT implementations to break ties among elements with equal timestamps. We will generally assume that metadata provides a strict ordering and ignore the details.

If the effects of all operations are delivered to all replicas eventually, the replicas will converge to the same state - assuming a quiescent period of time where no new operations are performed. This allows to eventually recover the consistency of the data type without giving away availability.

Specifications. The simplicity of the list data type with the API that we have described above allows for a somewhat simple conflict resolution strategy. Any strategy ordering conflicting concurrent insertions in a deterministic way will work. However, this is not true for many other CRDT implementations. It is therefore critical to provide the programmer with a clear, and precise, specification of the allowed behaviors of the data type under conflicts. Unfortunately this is not an easy task. Many times the programmer has no option but to read the implementation to understand how the metadata is used to resolve conflicts, for instance by reading the algorithms by Shapiro et al. [2011] (a case where the algorithms are particularly well documented). Recently Burckhardt [2014]; Burckhardt et al. [2014] have developed a formal framework where CRDTs and other weakly consistent systems can be specified. However, we consider that reading these specifications is far from trivial for the average programmer, let alone writing new specifications. Evidently, having a formal specification is a necessary step towards the verification of the implementations of CRDTs.

\footnotetext{
${ }^{3}$ We use $s_{0} \cdot s_{1}$ to denote the composition of sequences $s_{0}$ and $s_{1}$.

${ }^{4}$ We ignore here conflicts due to remove. They are discussed in Sec. 2.
} 
Simpler specifications, not simplistic specifications. It is important to remark at this point that while it is our goal to make the specification of CRDTs simpler, we believe that it is impossible to make them coincide with their sequential data type counterparts. Most CRDTs will exhibit, due to concurrency and consistency relaxations, behaviors that are not possible in the sequential version of the type they represent. A notable instance is the Multi-Valued-Register (MVR), which resolves conflicts arising from concurrent updates to the register by storing multiple values. Hence, a subsequent read operation to the register might return a set of values rather than a single value. This is certainly a behavior that is not possible for a "traditional" register, and in fact, one that the programmer must be aware of. Our goal is to accurately specify the behaviors of the CRDT, meaning that often times, different implementations of the same underlying data type (say a register) will have different specifications if their conflict resolution allows for different behaviours, for instance the Last-Writer-Wins (LWW) and the MVR registers which will be mentioned later.

Paper Contributions. Inspired by linearizability [Herlihy and Wing 1990] we propose a new consistency criterion for CRDTs, which we call Replication-Aware Linearizability (RA-linearizability). RA-linearizability both simplifies CRDT specifications, and allows us to give correctness proof strategies for these specifications. To satisfy RA-linearizability a data type must be so that any execution of a client interacting with an instance of the data type (1) should result in a state that can be obtained as a sequence (or linearization) of its updates - where we assume that all updates are executed sequentially- and (2) any operation reading the state of the data type instance should be justified by executing a sub-sequence of the above mentioned sequence of updates. For instance, for the RGA example, the state of the final list (when all updates are delivered) should be reachable by considering a sequence where all addAfter operations are executed sequentially. ${ }^{5}$ This definition shares some similarities with that of Perrin et al. [2014]. We address the main differences in Sec. 7.

Equipped with this criterion we show that many existing CRDTs are RA-linearizable. We provide both, their specification, and proofs showing that implementations respect the specification. We provide two different proof methodologies based on the structure of the conflict-resolution mechanism implemented by the CRDT. We categorize CRDT implementations into classes according to their conflict-resolution strategy. Encouragingly, most of the CRDTs by Shapiro et al. [2011] can be proved RA-linearizable.

Given that our criterion is inspired by linearizability, we consider if it also preserves the same compositionality properties, i.e. whether the composition of a set of RA-linearizable objects is also RA-linearizable. While we show that this is not true in general, we show that compositionality can be achieved when we concentrate to specific classes of conflict resolution as described above.

Finally, we have mechanized our methodologies to prove RA-linearizability. We use the verification tool Boogie [Barnett et al. 2005] to encode our specifications, CRDTs, and prove the correctness of the implementations (proof scripts are available at [boo [n. d.]]). To the best of our knowledge, the only other works that mechanize correctness proofs of CRDT implementations are [Gomes et al 2017; Zeller et al. 2014], which are frameworks directly carrying the proofs at a semantical level in Isabelle/HOL, and concentrating on proving Strong Eventual Consistency (SEC).

Finally, notice that the CISE tool [Najafzadeh et al. 2016] does not actually prove the data types, but rather invariants on top of them.

\section{OVERVIEW}

We give an informal description of our system model, and illustrate our contribution with two compelling CRDT implementations from [Attiya et al. 2016; Shapiro et al. 2011].

\footnotetext{
${ }^{5}$ We will come back to RGA to add remove in Sec. 2.
}

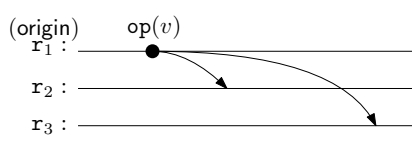

Fig. 1. System Model. 


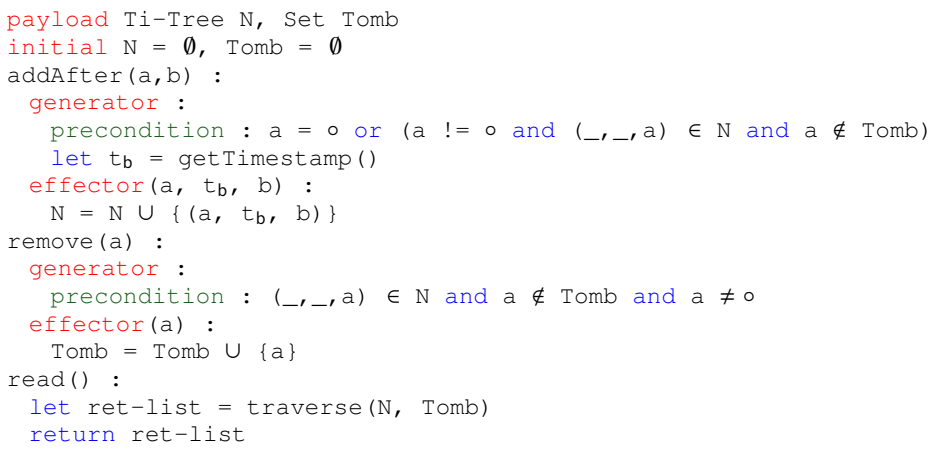

Listing 1. Replicated Growable Array (RGA) pseudo-code.

We assume that the system is comprised of multiple nodes in a network. We consider the implementation of CRDTs, and we focus on the behaviors of an an instance of the data type, generically called an object. We assume that objects are replicated among several replicas. Fig. 1 shows the execution of an operation $\circ \mathrm{p}(\mathrm{v})$ evolving as follows: (i) Firstly, a client issues a call to the object, connects to any one replica and performs the operation in that replica. We call that replica the origin. (ii) If the operation reads and updates the object state, the reading action is only performed at the origin. This part of the operation is called the generator (cf. [Shapiro et al. 2011]). Then, if the operation modifies the state e.g. addAfter for RGA - an update is generated to be executed in every replica. This part of the operation shall be called the effector. We assume that effectors are executed immediately at the origin. This is represented by the dot at the origin replica in Fig. 1. (iii) Finally, the effector is delivered to each replica, and their states are updated consequently, represented by the target of the arrows. This model corresponds to operation-based CRDTs. Our results also apply to state-based CRDTs, where replicas exchange states instead of operations (Sec. 6).

\subsection{RGA CRDT Implementation}

Listing 1 presents the code of RGA in a style following that of Shapiro et al. [2011] (a version of the RGA introduced in [Attiya et al. 2016]).

The keyword pay load declares the state used to represent the object: (akin to fields of a class in an object oriented language). a variable $\mathrm{N}$ of type $\mathrm{T} i-\mathrm{Tree}$, and a variable Tomb of type Set. We then find the definitions of the operations: addAfter, remove and read. The effectful operations addAfter and remove have two labels marked in red: generator and ef fector, corresponding to the reading and updating part of the operations as described above. Notice that the effector can use as arguments values produced by the generator. The precondition annotation indicates facts that are assumed about the state prior to the execution.

Reconsidering Fig. 1 the source of the arrows represents the execution of a generator jointly with the effector at replica $r_{1}$, and the target of the arrows represents the delivery and execution of the effector at replicas $r_{2}$ and $r_{3}$.

As it is common to many CRDT implementations, RGA replicas will use timestamps to keep track of causality between updates, effectively capturing when two updates are concurrent. Moreover, they will keep the information relating the causal order in which elements are added to the list. Provided with this causality information - or lack thereof-, the timestamps will be used to resolve conflicts in a deterministic way. Each replica maintains a Timestamp Tree (Ti-Tree) containing in every 


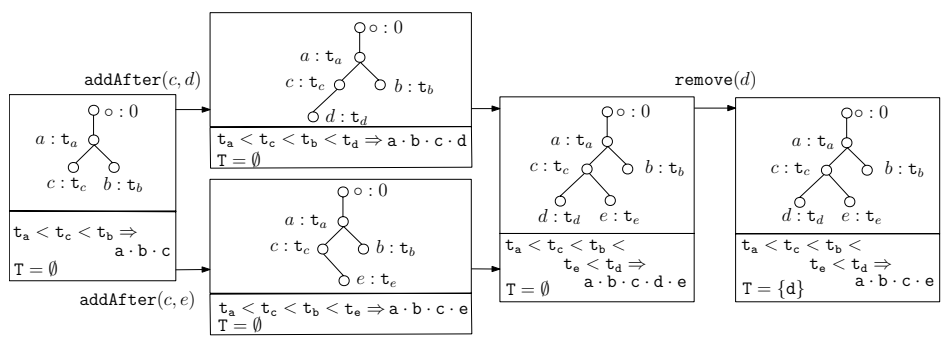

Fig. 2. Example of RGA conflict resolution.

tree node a pair with: the element added to the list (for instance the character $b$ ), and a timestamp associated to it $\left(t_{b}\right)$ used to resolve conflicts. We will encode the tree as a set of triples (corresponding to nodes) of the form $\left(a, t_{b}, b\right)$ representing an element $b$ in the tree with timestamp $t_{b}$ and whose parent is item a also present in the tree. The tree-ness property is ensured by construction.

The generator portion of addAfter $(a, b)$ has a precondition requiring a to exist in the tree before the insertion of $b$ (the data structure is initialized with a preexisting element $o$ ). The generator then samples a timestamp $t_{b}$ for $b$ which is assumed to be larger than any timestamp presently in the Ti-Tree $\mathrm{N}$ of the origin replica. ${ }^{6}$ The effector portion of addAfter $(a, b)$ adds the triple $\left(a, t_{b}, b\right)$ in the replica's own copy of $N$. This ensures that the tree structure is consistent with the causality of insertions in the data structure. A client of the object will only ever attempt to add an element after another element which it has already seen as mandated by the addAfter API. Hence, the parent node of any node was inserted before it, and is causally related to it. Similarly, nodes that are not related to each other on any path of the tree (eg. siblings) are not causally related. An example of such a tree is shown in the left most box of Fig. 2: elements $c$ and b were concurrently added after $a$, and a was added first after the initial element $\circ$.

From a $\mathrm{Ti}$-tree, we can obtain a list by traversing the tree in pre-order, with the proviso that siblings are ordered according to their timestamps with the highest timestamp visited first. The leftmost box in Fig. 2 shows a tree that results in the list $a \cdot b \cdot c$ assuming the timestamp order $t_{a}<t_{c}<t_{b}$.

Consider now two concurrent operations: addAfter $(c, d)$ and addAfter $(c, e)$ executing in two different replicas starting both with the state depicted on the left of Fig. 2. Following Listing 1 we obtain the trees in the second column of Fig. 2 where we assume that the top tree is the result of addAfter $(c, d)$ in one of the replicas, and the bottom tree is the result of executing addAfter $(C, e)$ in the other. Then, the two trees result in different lists in each replica before the operations are mutually propagated. In the third column of Fig. 2 we obtain the result of propagating the operations between the replicas - indeed the propagation of any of the operations to any of the replicas yields the same result, ensured by the commutativity of CRDTs. It is clear now that the result of the list is $a \cdot b \cdot c \cdot d \cdot e$.

We have so far ignored remove. Consider the case where a replica executes addAfter $(a, b)$ on a replica while another one executes remove (a). If the effector of remove (a) reaches every replica after the effector of addAfter $(a, b)$ there is no problem since the semantics is clear: the element $a$ is removed after the element $b$ has been added. However, if the operations reach some replica in opposite order (recall that they are concurrent) there is a problem since the precondition of the effector of addAfter $(a, b)$ requires that the element a be present in the Ti-tree of the replica. To avoid this kind of conflict, rendering the operations commutative, RGA does not really remove elements from the $\mathrm{Ti}$-tree. Instead, an additional data structure called a tombstone is used

\footnotetext{
${ }^{6}$ Also, $t_{b}$ cannot be sampled by another replica (as we discussed in Sec. 1 this can be ensured by tagging the timestamps with replica identifiers).
} 


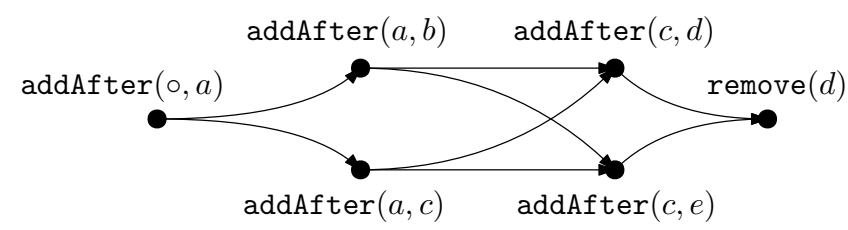

Fig. 3. A history for the RGA object.

to keep track of elements that have been conceptually erased and should not be considered when reading the list. Here, the marking of tombstones is a set Tomb of elements. The last column of Fig. 2 shows the result of a remove operation.

The method read performs the pre-order traversal explained before, where all elements in the tombstone Tomb are omitted. In each of the boxes of Fig. 2 the list shown represents the result of a read operation in the state depicted.

Operations, histories and linearizability. We consider an abstract view of executions of a CRDT object called a history. Informally a history is a set of operations with a partial order representing the ordering constraints imposed on the execution of each operation. We represent the execution of an operation with a label of the form $\mathrm{m}(a) \Rightarrow b$ representing a call to method $\mathrm{m}$ with arguments $a$ and returning the value $b$. When the values are unimportant we shall use the meta-variable $\ell$ to denote a label. The partial order mentioned above represents the visibility relation among operations. We say that an operation with label $\ell_{1}$ is visible to an operation with label $\ell_{2}$ if at the time when $\ell_{2}$ was executed at the origin replica, the effects of $\ell_{1}$ had been applied in the state of the replica executing $\ell_{2}$. A history is a pair $(\mathrm{L}, \prec)$ containing a set of labels $L$ and a visibility relation $\prec$ between labels. The history of the execution in Fig. 2 is presented in Fig. 3. Each node represents a label and arrows represent that the operation at the source of the arrow is visible to the operation at the target. Since we assume that visibility is transitive we ignore redundant arrows.

A similar notion of history is used in the context of linearizability [Herlihy and Wing 1990]. The only difference is that the order $<$ relates two operations the first of which returns before the other one started. A history $(\mathrm{L}, \prec)$ is called linearizable if there exists a sequential history $\left(\mathrm{L}, \prec_{\text {seq }}\right)\left(\prec_{\text {seq }}\right.$ is a total order), called linearization, s.t. $\left(\mathrm{L}, \prec_{\text {seq }}\right.$ ) is a valid execution, and $\prec \subseteq \prec_{\text {seq }}$.

CRDTs are not linearizable since operations are propagated lazily, so two replicas can see noncoinciding sets of operations. We relax linearizability to adapt it to CRDTs as follows: (1) we require that the sequential history be consistent with the visibility relation among operations instead of the returns-before order, and (2) operations that only read the state of the object are allowed to see a sub-sequence of the linearization, instead of the whole prefix as in the case of linearizability. (We will discuss an additional relaxation in Sec. 2.2).

Intuition of RGA RA-linearizability. To simplify, consider the linearization of two concurrent operations adding after a common element: $\operatorname{addAfter}(\mathrm{a}, \mathrm{b})$ and addAfter $(\mathrm{a}, \mathrm{c})$. This example corresponds to the history shown in the first three nodes of Fig. 3 from left to right. Because these operations are concurrent they are not related by visibility so our criterion allows for any ordering among them. Let us show that these operations can always be ordered in a way that the result of future reads will match this ordering. From the previous explanation we know that the order between $b$ and $c$ in the resulting list will be determined by their corresponding timestamps $\left(t_{b}\right.$ and $\left.t_{c}\right)$. Assuming that the ordering is that given in the tree of the first column of Fig. 2, we know that we can order the operations as addAfter $(a, c)$ followed by addAfter $(a, b)$ which when executed sequentially obviously results in $a \cdot b \cdot c$ as shown. The timestamp metadata of RGA gives us a strategy to build the operation sequence that corresponds to a sequential specification. A concrete linearization of these operations is: 


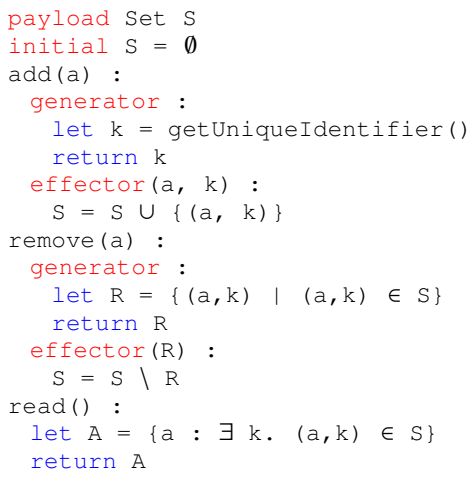

Listing 2. Pseudo-code of the OR-Set CRDT.

$$
\operatorname{addAfter}(\circ, a) \cdot \operatorname{addAfter}(a, c) \cdot \operatorname{addAfter}(a, b)
$$

Unfortunately this simple linearization strategy is not always applicable. Consider now a similar case where after issuing the addAfter operations the replicas attempt to immediately read the state. As explained in Fig. 2, a possible behavior is that the first replica returns $\circ \cdot a \cdot b$ while the second returns $\circ \cdot a \cdot c$. If we consider the linearization given above, the result $\circ \cdot a \cdot b$ is not possible, since $c$ was added before $b$ was added. The problem here is that the replica executing this read has not yet seen the effect of addAfter $(a, c)$. To overcome this problem we allow methods that read the state to see a sub-sequence of the global linearization. Thus, we can consider the sequence

$$
\begin{array}{r}
\operatorname{addAfter}(\circ, a) \cdot \operatorname{addAfter}(a, c) \cdot \operatorname{read}() \Rightarrow(\circ \cdot a \cdot c) \cdot \\
\operatorname{addAfter}(a, b) \cdot \operatorname{read}() \Rightarrow(\circ \cdot a \cdot b)
\end{array}
$$

where the last read ignores the red label addAfter $(a, c)$. These are only two cases of conflicting concurrent operations, in Sec. 4 we show that all operations can be ordered such that they correspond to a sequential execution thereof.

\subsection{OR-Set CRDT Implementation}

The Observed-Remove Set (OR-Set) [Shapiro et al. 2011] implements a set with operations: add (a), remove (a), read ( ). The code of OR-Set is shown in Listing 2 (we assume return values for add (a) and remove (a) for technical reasons).

Although the meaning of these methods is self-evident from their names, the results of conflicting concurrent operations is not evident. Consider for example the case where two replicas add a certain element $a$ and then one of them removes that element. If we consider an interleaving based execution of these operations there are two options depending on the interleaving: i) If remove (a) is the last operation

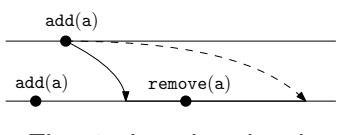

Fig. 4. Interleaving-based Set. then the expected set is empty, since the two consecutive add (a) are idempotent, and the remove would remove the only occurrence of a. This interleaving is the one depicted with solid arrows in Fig. 4. ii) On the other hand, if the operation add (a) of the non-removing process comes last, as depicted with the dashed arrows in Fig. 4, the final set could contain the element a. As we have explained before, the operations can arrive in different orders to different replicas. To guarantee convergence, OR-Set must ensure that regardless of the ordering, the resulting set will be the same. To that end, OR-Set add operations will tag each added element with a unique identifier. Then, a remove operation will only remove the element-identifier pairs which has already seen. For instance, in the case (ii) above, the remove of a will only remove the element that has been previously added 


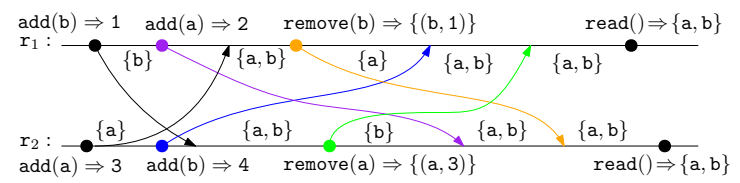

(a) OR-Set non-linearizable execution. Each line represents operations issued to the same replica.

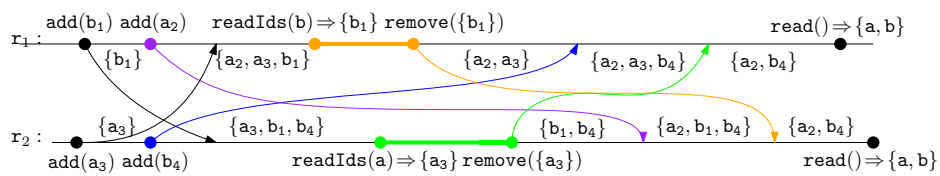

(b) Label rewriting of an OR-Set execution. Pairs $(a, k)$ of an element $a$ and identifier $k$ are written as $a_{k}$.

Fig. 5. OR-Set Linearizability vs. RA-linearizability.

by the same replica, since this item has been observed by the remove operation - and thus its identifier is known to it. The concurrent add (a) operation will have an identifier that has not been observed by the remove Therefore the item will not be removed, even if the effectors of the two adds are performed in a replica before the effector of the remove.

Intuition of OR-Set Linearizability. It is easy to find examples where the implementation of ORSet can produce executions that cannot be justified by the standard definition of linearizability (even with the relaxations discussed in Sec. 2.1) assuming a standard Set specification. Fig. 5a shows one such example. Clearly any linearization of the visibility relation in this execution should order the add and remove updates before the read queries, and the linearization of the updates should end with a remove. Therefore, the final set returned by each of the two read queries should have at most one element (the read queries see all the updates in the execution), contrary to their return value in this execution.

This execution shows that the remove operation behaves as both a query (observing a certain number of adds of the element to be removed) and an update (by removing said observed elements). To cope with such cases, we will consider in our definition that query-update operations can be split into a query part corresponding to the generator, which only reads the state - and hence is allowed to see a sub-sequence of the linearization of updates - and an update part corresponding to the effector which will use the results of the prior query. For instance, remove will be split into a query part readIds where only the elements visible at the time of the remove are selected, and an update part remove where only those elements selected are erased. Any identifier not in the set returned by readIds will remain in the set after the update part of remove. Evidently, this requires some mechanism for "marking" the adds that are concerned. We will consider that each add has a unique identifier. Fig. 5b shows this rewriting. The result of the rewriting admits a linearization consistent with the specification of Set, as explained above.

\section{REPLICATION-AWARE LINEARIZABILITY}

In this section we formalize the intuitions developed in Sec. 2. We define the semantics of CRDT objects ( $\$ 3.1$ ), specifications ( $(3.2)$, and our notion of RA-linearizability ( $\$ 3.3)$. For lack of space, our formalization focuses only on operation-based CRDTs. However, the notion of RA-linearizability applies to state-based CRDTs as well (see Section 6). 


\subsection{The Semantics of CRDT objects}

To formalize the semantics of CRDT objects and our correctness criterion we use several semantic domains summarized in Fig. 6. We let $o \in \mathbb{O}$ be a CRDT object in the set of objects $\mathbb{O}$. Similarly, $r \in \mathbb{R}$ is a replica in the set of replicas $\mathbb{R}$. We consider a set of method names $m \in \mathbb{M}$, and that each method has a number of arguments and a return value sampled from a data domain $\mathbb{D}$. We assume that the domain contains a special value $\perp \in \mathbb{D}$ used to represent the absence of a value (for instance the return type of procedures). Furthermore, we ignore typing issues which should be addressed by an underlying programming language. Also, some methods, e.g., the method addAfter of the RGA object, generate timestamps from a totally-ordered domain $\mathbb{T}$. We will use operation labels of the form $0 . \mathrm{m}(a) \stackrel{i, t s}{\Rightarrow} b$ to represent the call of a method $\mathrm{m} \in \mathbb{M}$ of object $\mathrm{o} \in \mathbb{O}$, with argument $a \in \mathbb{D}$, resulting in the value $b \in \mathbb{D}$, and generating the timestamp $t s$. Since there might be multiple calls to the same method with the same arguments and result, labels are tagged with a unique identifier $i$. We may omit the object o, the identifier $i$, the timestamp $t s$, or the return value $b$ when they are not important. The order relation on $\mathbb{T}$ is denoted by $<$. Abusing notations, we assume that the set $\mathbb{T}$ contains a distinguished minimal element $\perp$ which we shall use for operations that do not generate a timestamp such as the method remove of RGA. The timestamp ts of a label $\ell=0 \cdot \mathrm{m}(a) \stackrel{i, t s}{\Rightarrow} b$ is denoted $\operatorname{ts}(\ell)$. We will ignore identifiers when unambiguous. The set of all operation labels is denoted by $\mathbb{L}$.

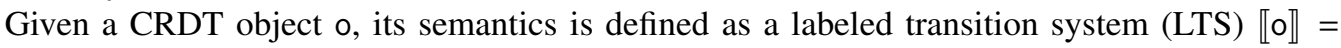
$\left(\mathrm{GC}, \mathbb{A}, \mathrm{gc}_{0}, \rightarrow\right.$ ), where $\mathrm{GC}$ is a set of global configurations, $\mathbb{A}$ is the set of transition labels called actions, $\mathrm{gc}_{0}$ is the initial configuration, and $\rightarrow \subseteq \mathrm{GC} \times \mathbb{A} \times \mathrm{GC}$ is the transition relation. We use the action $\operatorname{gen}_{r}(\ell)$ to label the generator of $\ell$ when executed at replica $r$, and eff $r(\ell)$ to label the effector of $\ell$ when executed at $r$. For readability, we use gc $\stackrel{a}{\rightarrow} \mathrm{gc}^{\prime}$ to denote a transition $\left(\mathrm{gc}, a, \mathrm{gc}^{\prime}\right) \in \rightarrow$.

Our semantics assumes the following two properties of the propagation of effectors: (i) the effector of each operation is applied exactly once at each replica, and (ii) if the effector of operation $\ell_{1}$ is applied at the origin replica of $\ell_{2}$ before $\ell_{2}$ happens, then for every replica $r$, the effector of $\ell_{2}$ will be applied only after the effector of $\ell_{1}$ has already been applied. These are commonly referred to as causal delivery. We assume causal delivery because our formalization focuses on operation-based CRDTs. However, the notion of RA-linearizability and the compositionality results in Section 5 apply to state-based CRDTs as well, even if the network infrastructure doesn't satisfy causal delivery.

A global configuration (G, vis, DS) is a "snapshot" of the system that records all the operations that have been executed. $G \in[\mathbb{R} \rightarrow \mathrm{LC}]^{7}$ stores the local configuration of each replica (LC denotes the set of local configurations). A local configuration $(\mathrm{L}, \sigma)$ contains the state $\sigma$ of a replica and the set $L$ of labels of operations that originate at this replica, or whose effectors have been executed (or applied) at this replica. When $\ell \in \mathrm{L}$, we say that $\ell$ is visible to the replica or that the replica sees $\ell$. The set of replica states $\sigma$ is denoted by $\Sigma$. The relation vis $\subseteq \mathcal{P}(\mathbb{L} \times \mathbb{L})$ is the visibility relation between operations, i.e., $\left(\ell_{1}, \ell_{2}\right) \in$ vis, where $\ell_{2}$ is an operation originated at a replica $r$, if the effector of $\ell_{1}$ was executed at $r$ before $\ell_{2}$ was executed. When $\left(\ell_{1}, \ell_{2}\right) \in$ vis, we say that $\ell_{1}$ is visible to $\ell_{2}$, or that $\ell_{2}$ sees $\ell_{1}$. As it will be clear from the definition of the transition relation, vis is a strict partial order. Finally, DS $\in[\mathbb{L} \rightarrow \Delta]$ associates to each operation label $\ell \in \mathrm{L}$ an effector $\delta \in[\Sigma \rightarrow \Sigma]$, which is the replica state transformer generated when the operation was executed at the origin replica ( $\Delta$ denotes the set of effectors). For some fixed initial replica state $\sigma_{0}$, the initial global configuration is defined by $\mathrm{gc}_{0}=\left(\mathrm{G}_{0}, \emptyset, \emptyset\right) \in \mathrm{GC}$, where $\mathrm{G}_{0}$ maps each replica $r$ into $\left(\emptyset, \sigma_{0}\right)$.

The transition relation between global configurations is defined in Fig. 7. The first rule describes a replica $r$ in state $\sigma$ executing an invocation of method $\mathrm{m}$ with argument $a$. We use a function $\theta$ to

${ }^{7}$ We use $[A \rightarrow B]$ to denote the set of total functions from $A$ to $B$. 


$$
\begin{aligned}
& \circ \in \mathbb{O} \text { CRDT Objects } a, b \in \mathbb{D} \text { Data } \\
& r \in \mathbb{R} \text { Replicas ts } \in \mathbb{T} \text { Timestamps } \\
& \mathrm{m} \in \mathbb{M} \text { Methods } \quad \text { L } \subseteq \mathbb{L} \text { Label Set } \\
& \ell \equiv \operatorname{o.m}(a) \stackrel{i, t s}{\Rightarrow} b \quad \in \quad \mathbb{L} \quad \text { Operation Label }
\end{aligned}
$$

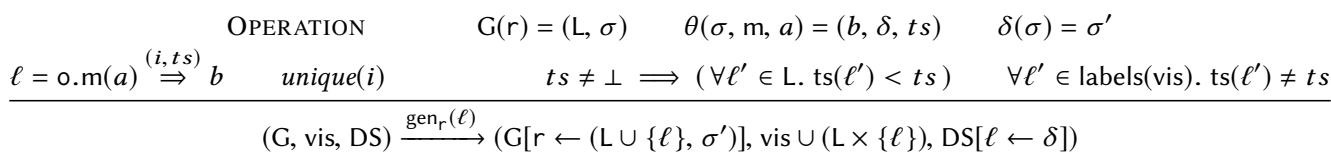

$$
\begin{aligned}
& \begin{array}{ccrcr}
\text { EFFECTOR } & \mathrm{G}(\mathrm{r})=(\mathrm{L}, \sigma) \quad \ell \in \min _{\text {vis }}(\text { labels }(\text { vis }) \backslash \mathrm{L}) & \mathrm{DS}(\ell)=\delta & \delta(\sigma)=\sigma^{\prime}
\end{array}
\end{aligned}
$$

Fig. 7. Operational Semantics of CRDTs. $C[a \leftarrow b]$ denotes the in-place update of element $a$ of the domain of $C$ with value $b$; unique $(i)$ to ensure that $i$ is a unique identifier; and labels(vis $)=\{\ell$ : $\exists \ell^{\prime} .\left(\ell, \ell^{\prime}\right) \in \operatorname{vis} \vee\left(\ell^{\prime}, \ell\right) \in$ vis $\}$.

represent the behavior of the generators of all methods collectively (the code under the generator labels), i.e., $\theta(\sigma, \mathrm{m}, a)$ stands for applying the generator of $\mathrm{m}$ with argument $a$ on the replica state $\sigma$. Therefore, this transition applies the suitable generator, which results in a return value $b$, an effector state transformer $\delta$ to be applied on all replicas, and possibly, a timestamp $t s$. We have $t s=\perp$ for methods that don't generate timestamps. We assume that timestamps are consistent with the visibility relation vis, i.e., the timestamp $t s$ generated by $\theta$ is strictly larger than all the timestamps of operations visible to $r$, and that each timestamp can be generated only once. The association between the label $\ell$ corresponding to this invocation and the effector $\delta$ is recorded in the DS component of the new global configuration. We say that the effector $\delta$ is produced by the operation $\ell$. The local configuration ( $\mathrm{L}, \sigma)$ of $r$ is changed by applying the effector $\delta$ on the state $\sigma$, resulting in a new state $\sigma^{\prime}$, and adding $\ell$ to the set of labels L. Finally, the visibility relation vis is changed to record the fact that the effectors of all operations in $L$ have been applied before $\ell$. This transition is labeled by gen $_{r}(\ell)$ where $\ell$ is the label of this invocation. We may ignore the index $r$ when it is not important.

The second rule describes a replica $r$ in state $\sigma$ executing the effector $\delta$ that corresponds to an operation $\ell$ originated in a different replica. ${ }^{8}$ The rule requires that $\delta$ is an effector of a label that has not yet been applied at $r$ (i.e., its corresponding label is not in the L component of $r$ 's configuration) and moreover, that it is a minimal one with respect to the order vis among such effectors, i.e., there exists no $\ell^{\prime} \notin \mathrm{L}$ such that $\left(\ell^{\prime}, \ell\right) \in$ vis. This transition results in modifying the state of $r$ to $\delta(\sigma)$ and adding $\ell$ to the set of operations whose effectors have been executed by $r$. Note that these transition rules preserve the fact that vis is a strict partial order. This transition is labeled by $\operatorname{eff}_{r}(\ell)$.

In what follows it will be useful to distinguish query (or pure) methods which do not modify the state from state-modifying methods, which we shall call updates (or effectful). We say that a method $\mathrm{m} \in \mathbb{M}$ is a query if it always results (by applying the generator) in an identity effector $\delta$ (i.e. $\delta(\sigma)=\sigma$ for all replica states $\sigma$ ). We shall call an update any method $\mathrm{m}$ which is not a query that is, whose effectors are not the identity function - and whose resulting effector and return value do not depend on the initial state $\sigma$ of the origin replica. That is, its behavior is fully determined by its arguments. More formally, assuming a functional equivalence relation $\equiv$ between effectors that relates any two effectors that have the same effect (modulo the values of timestamps or unique identifiers) $\mathrm{m}$ is called an update when $\left.\left.\theta(\sigma, \mathrm{m}, a)\right|_{2} \equiv \theta\left(\sigma^{\prime}, \mathrm{m}, a\right)\right|_{2}$, for every $a \in \mathbb{D}$ and two states $\sigma, \sigma^{\prime} \in \Sigma$ (for a tuple $x,\left.x\right|_{k}$ denotes the projection of $x$ on the $k$-th component). A method $\mathrm{m}$ which

\footnotetext{
${ }^{8}$ This rule implies that we could simplify the first rule by not performing the effector immediately, but in general we assume no interleavings of operations within a single replica.
}

Proc. ACM Program. Lang., Vol. 1, No. CONF, Article 1. Publication date: January 2018. 
is not a query nor an update is called a query-update. (It generates an effector which is not the identity function, and whose effect depends on the local state of the replica at which the invocation of $m$ originated.) For instance, the methods addAfter and remove of RGA, and add of OR-Set, are updates, the method remove of OR-Set is a query-update, and the read methods of both the RGA and the OR-Set are queries. We denote by Queries, Updates, and Query-Updates, the sets of operation labels o.m $(a) \stackrel{i, t s}{\Rightarrow} b$ where $\mathrm{m}$ is a query, an update, or query-update respectively. We shall call them query, update, and query-update labels,respectively.

An execution of the object $\mathrm{o}$ is a sequence of transitions $\mathrm{gc}_{0} \stackrel{a_{0}}{\longrightarrow} \mathrm{gc}_{1} \stackrel{a_{1}}{\longrightarrow} \ldots$. A trace tr is the sequence of actions $a_{0} \cdot a_{1} \ldots$ labeling the transitions of an execution. The set of traces of an object $\mathrm{O}$ is denoted by $\operatorname{Tr}(\mathrm{o})$. A history is a pair (L, vis) where vis $\subseteq \mathrm{L} \times \mathrm{L}$ is an acyclic relation over the set of labels L. Given an execution $e$ ending in a global configuration (G, vis, DS), the history of $e$, denoted by $h(e)$, is the pair (labels(vis), vis). Note that the relation vis is a strict partial order in this case. We will later allow a more general notion of history in order to deal with object compositions (see Sec. 5). Also, the history of a trace $t r$, denoted by $h(t r)$, is the history of the execution that corresponds to $t r$. The set of histories $\mathbb{H}$ ist(o) of an object o is the set of histories $h$ of an execution $e$ of o. A pictorial representation of an execution (trace) can be found in Fig. 5a while an example of a history can be found in Fig. 3.

\subsection{Sequential Specifications}

RA-linearizability provides an explanation for concurrent executions of CRDT objects in the form of linearizations, which can be constrained using standard sequential specifications.

Definition 3.1 (Sequential Specification). A sequential specification (specification, for short) Spec is a set of tuples ( $\mathrm{L}$, seq), where $\mathrm{L}$ is a set of labels and seq is a sequence including all the labels in $\mathrm{L}$.

To describe sequential specifications in a succinct way we will provide an operational description. To that end, we will associate to specifications a notion of abstract state, which we shall generally denote by $\phi$ and its domain shall be denoted by $\Phi$. Then, to each valid label $\ell$ we will associate a transition relation $\phi \stackrel{\ell}{\hookrightarrow} \phi^{\prime}$ which, given an abstract state $\phi$ and provided that the label $\ell$ can be applied in $\phi$, produces a new abstract state $\phi^{\prime}$. In the specific case where the label $\ell$ assumes a certain precondition pre over the initial abstract state $\phi$ we will use Hoare-style preconditions and write $(\phi \mid \operatorname{pre}(\phi)) \stackrel{\ell}{\hookrightarrow} \phi^{\prime}$. In this way, a sequential specification is the set of labels that are accepted by the successive application of the transition relation starting from some given initial state $\phi_{0}$.

To illustrate the definition we provide the sequential specification of a very simple counter object, as well as the RGA and OR-Set objects described before.

Example 3.2 (Sequential Specification of a Counter). In this case the state domain is $\Phi=\mathbb{Z}$, that is the state will be an integer, and the transitions are given as follows:

$$
k \stackrel{\operatorname{inc}() \Rightarrow}{\longrightarrow} k+1 \quad k \stackrel{\operatorname{dec}() \Rightarrow}{\longrightarrow} k-1 \quad k \stackrel{\operatorname{read}() \Rightarrow k}{\longrightarrow} k
$$

Example 3.3 (Sequential Specification of RGA). Each abstract state $\phi=(l, T)$ contains a sequence $l$ of elements of a given type and a set $T$ of elements appearing in the list. The element $l$ is the list of all input values, whether already removed or not; while $T$ stores the removed values and is used as tombstone set. The sequential specification $\operatorname{Spec}(\mathrm{RGA})$ of list with add-after interface is defined by:

$$
\begin{aligned}
&\left(\left(l_{1} \cdot b \cdot l_{2}, T\right) \mid a \text { fresh }\right) \underset{((l, T) \mid b \in l \text { and } b \neq 0)}{\stackrel{\operatorname{addAfter}(b, a)}{\operatorname{remove}(b)}}\left(l_{1} \cdot b \cdot a \cdot l_{2}, T\right) \\
&(l, T) \stackrel{\operatorname{read}() \Rightarrow(l / T)}{\longrightarrow}(l, T \cup\{b\}) \\
& \stackrel{(l, T)}{\longrightarrow}
\end{aligned}
$$


where we denote by $l / T$ the list resulting from removing all elements of $T$ from $l$. The method addAfter $(b, a)$ puts $a$ immediately after $b$ in $l$, assuming that each value is put into list at most once. Method remove $(b)$ adds $b$ into $T$, hence removing $b$ from the list for subsequent calls to the read method. Finally $\operatorname{read}() \Rightarrow s$ returns the list content excluding any element appearing in $T$. Assume that the initial value of list is $(\circ, \emptyset)$, and $\circ$ is never removed. We will sometimes ignore the value $\circ$ from the return of read.

Example 3.4 (Sequential Specification of OR-Set). As explained in Fig. 5b, the fact that the OR-Set remove method is a query-update induces a rewriting of the operation labels in a history. This rewriting introduces update operations $\operatorname{add}(a, i d)$, for some identifier $i d$, instead of simply add $(a)$, and remove $(S)$, for some set $S$ of pairs element-identifier, instead of remove $(a)$, and a new query operation readIds $(a)$ that returns a set of pairs element-identifier. These operations are specified as follows. The abstract state $\phi$ is a set of tuples $(a, i d)$, where $a$ is a data and $i d$ is a identifier. The sequential specification $\operatorname{Spec}(\mathrm{OR}-\mathrm{Set})$ of OR-Set is given by the transitions:

$$
\begin{aligned}
& \phi \stackrel{\text { readIds }(a) \Rightarrow S}{\stackrel{\text { remove }(S)}{\longrightarrow}} \phi \quad[S=\{(a, i d) \mid(a, i d) \in \phi\}] \\
& \phi \stackrel{\text { remove }(S)}{\longrightarrow} \phi \backslash S \\
& (\phi \mid(a, i d) \notin \phi) \quad \stackrel{\operatorname{add}(a, i d)}{\longleftrightarrow} \phi \cup\{(a, i d)\} \\
& \phi \stackrel{\operatorname{read}(a) \Rightarrow A}{\longrightarrow} \phi \quad[A=\{a \mid \exists i d,(a, i d) \in \phi\}]
\end{aligned}
$$

Here readIds $(a) \Rightarrow S$ returns the set of pairs with data $a$, remove $(S)$ removes $S$ from the abstract state, $\operatorname{add}(a, i d)$ puts $\{(a, i d)\}$ into the abstract state, and $\operatorname{read}() \Rightarrow A$ returns the value of the OR-Set.

This definition of specification of an object will be extended to a set of objects in Sec. 5. Another important aspect of specifications is whether they are deterministic or not. For instance the Wooki CRDT [Weiss et al. 2007] is a list-like object that provides a method addBetween $(a, b, c)$ which inserts $b$ between $a$ and $c$. In contrast with RGA, where the method addAfter $(a, b)$ adds the element $b$ immediately after $a$, in Wooki there are many possible positions where to insert $b$ if $a$ and $c$ are not adjacent. In this case, to allow for any deterministic resolution mechanism our specifications shall be non-deterministic. This non-determinism in the specification has to be deterministically resolved by the implementations to ensure convergence.

\subsection{Definition of Replication-Aware Linearizability}

We now provide the definition of RA-linearizability which characterizes histories of CRDT objects. To simplify the presentation, we consider first the case where all the labels in the history are either queries or updates (query-updates are considered later). The intuition of RA-linearizability is that there is a global sequence (or linearization) of the update operations in an execution which can produce the state of each replica when all the updates are visible to them. In intermediate steps, any replica state should be the result of applying a sub-sequence of updates of this global sequence. This is because replicas may see a subset of the updates performed up to some moment. Therefore, each query should be justified by considering the sub-sequence of the global sequence restricted to the updates that are visible to that query. To be precise:

Definition 3.5. A history $h=(\mathrm{L}, \mathrm{vis})$ with $\mathrm{L} \subseteq$ Queries $\uplus$ Updates is RA-linearizable w.r.t. a sequential specification Spec, if there exists a sequence $(\mathrm{L}, \mathrm{seq})$ - where we remark that the set of labels are identical - such that:

(i) seq is consistent with vis, that is: vis $\cup$ seq is acyclic,

(ii) the projection of seq to updates is admitted by Spec, i.e. seq $\downarrow_{\text {Updates }} \in \mathrm{Spec}$, where we denote by seq $\downarrow_{S}$ the restriction of the order seq to the set $S$, and 
(iii) for each query $\ell_{\mathrm{qr}} \in \mathrm{L}$, the sub-sequence of updates visible to $\ell_{\mathrm{qr}}$ together with $\ell_{\mathrm{qr}}$ is itself admitted by Spec, i.e., seq $\downarrow_{\mathrm{vis}^{-1}\left(\ell_{\mathrm{qr}}\right) \cap \text { Updates }} \cdot \ell_{\mathrm{qr}} \in \mathrm{Spec}$.

We say that (L, seq) is an RA-linearization of $h$ w.r.t. Spec.

In a nutshell, this definition requires that for a given history, there exists a specification sequence such that (i) the set of labels are the same and the order in the sequence is consistent with the visibility order of the history, that (ii) when restricted to update operations - that is all the updates -, the sequence belongs to the specification, and that (iii) every query operation can be justified by the specification based only on the updates that precede it in the sequence and that are visible to it. With this definition in mind it is not hard to check that the sequences of operations provided in Sec. 2.1 and 2.2 are RA-linearizations. Another example constructing a linearization step by step is shown under the label "timestamp-order linearizations" of Fig. 8.

We now consider the case where histories include query-updates. In such case, we apply Definition 3.5 on a rewriting of the original history where each query-update is decomposed into a label representing the generator and another label representing the effector. As shown in Fig. 5b this rewriting may introduce new labels of operations that have been added to the specification of the data type to provide specifications with no query-update operations. A mapping $\gamma: \mathbb{L} \rightarrow \mathbb{L} \leq 2$, where $\mathbb{L}^{\leq 2}$ is the set of labels and pairs of labels in $\mathbb{L}$, is called a query-update rewriting. We assume that every query or update label is mapped by $\gamma$ to a singleton and that the $\gamma$ image of such a label preserves its status, i.e., $\gamma(\ell)$ is a query, resp., update, whenever $\ell$ is a query, resp., update. Also, query-updates labels $\ell$ are mapped to pairs $\gamma(\ell)=\left(\ell_{1}, \ell_{2}\right)$ where $\ell_{1}$ is a query and $\ell_{2}$ is an update These assumptions are important when applying Definition 3.5 on the rewriting of a history, since this definition relies on a partitioning of the labels into queries and updates. For a history $h=(\mathrm{L}$, vis), its $\gamma$-rewriting is a history $\gamma(h)=\left(\mathrm{L}^{\prime}\right.$, vis' $)$ where

- $\mathrm{L}^{\prime}$ is obtained by replacing each label $\ell$ in $\mathrm{L}$ with $\gamma(\ell)$ (a label may be replaced by two labels),

- whenever a (query-update) label $\ell$ is mapped by $\gamma$ to a pair $\left(\ell_{1}, \ell_{2}\right)$, we have that the query is ordered before the update, formally $\left(\ell_{1}, \ell_{2}\right) \in$ vis' $^{\prime}$,

- vis' preserves the order between labels which are mapped to singletons, and for any queryupdate label $\ell$ mapped to a pair $\left(\ell_{1}, \ell_{2}\right)$, the query $\ell_{1}$ sees exactly the same set of operations as $\ell$ and any operation which saw $\ell$ must see $\ell_{2}$. Formally, whenever $\left(\ell, \ell^{\prime}\right) \in$ vis we have that $\left(\operatorname{upd}(\gamma(\ell)), \operatorname{qry}\left(\gamma\left(\ell^{\prime}\right)\right)\right) \in \operatorname{vis}^{\prime}$, where for a label $\ell, \operatorname{qry}(\gamma(\ell))(\operatorname{resp}$., $\operatorname{upd}(\gamma(\ell)))$, is $\gamma(\ell)$ when $\gamma(\ell)$ is a singleton, or its first (resp., second) component when $\gamma(\ell)$ is a pair.

Example 3.6 (Query-Update Rewriting of OR-Set). As shown in Fig. 5b, the query-update rewriting for OR-Set is defined by: $\gamma(\operatorname{add}(a) \Rightarrow k)=\operatorname{add}(a, k), \gamma(\operatorname{read}() \Rightarrow \mathrm{A})=\operatorname{read}() \Rightarrow \mathrm{A}$, and $\gamma(\operatorname{remove}(a) \Rightarrow R)=(\operatorname{readIds}(a) \Rightarrow R, \operatorname{remove}(R))$.

The following extends Definition 3.5 to arbitrary histories using the rewriting defined above.

Definition 3.7 (Replication-Aware Linearizability). A history $h=(\mathrm{L}, \mathrm{vis})$ is RA-linearizable w.r.t. Spec, if there exists a query-update rewriting $\gamma$ s.t. $\gamma(h)$ is RA-linearizable w.r.t. Spec. An RA-linearization w.r.t. Spec of $\gamma(h)$ is called an RA-linearization w.r.t. Spec and $\gamma$ of $h$.

A set $H$ of histories is called RA-linearizable w.r.t. Spec when each $h \in H$ is RA-linearizable w.r.t. Spec. A data type implementation is RA-linearizable w.r.t. Spec if for any object o of the data type, $\mathbb{H}$ ist(o) is linearizable w.r.t. Spec.

Reasoning with specifications. To illustrate the benefit of using RA-linearizability let us consider a simple system where two replicas execute a sequence of operations on a shared OR-Set object:

$\operatorname{add}(a) ; \operatorname{rem}(a) ; X=\operatorname{read}() \| \operatorname{add}(a) ; Y=\operatorname{read}()$

Proc. ACM Program. Lang., Vol. 1, No. CONF, Article 1. Publication date: January 2018. 
We are interested in checking that the following post-condition holds after the execution of these operations:

$$
a \in X \Rightarrow a \in Y
$$

Rewriting the program according to the specification of OR-Set discussed before, we obtain the following, where the variable $\mathrm{R}$ represents the set of value timestamp pairs observed by the readlds operation as defined by the rewriting:

$$
\begin{gathered}
\left.\left[\begin{array}{l}
\operatorname{add}\left(\mathrm{a}, \mathrm{i}_{1}\right) ; \\
\operatorname{readIds}(\mathrm{a}) \Rightarrow \mathrm{R} ; \\
\operatorname{rem}(\mathrm{R}) ; \\
\mathrm{X}=\operatorname{read}() ; \\
\left\{\mathrm{a} \in \mathrm{X} \Rightarrow\left(a, i_{2}\right) \notin \mathrm{R}\right\}
\end{array}\right] \| \begin{array}{l}
\operatorname{add}\left(\mathrm{a}, \mathrm{i}_{2}\right) ; \\
\mathrm{Y}=\operatorname{read}() ; \\
\left\{\left(a, i_{2}\right) \notin \mathrm{R} \Rightarrow \mathrm{a} \in \mathrm{Y}\right\}
\end{array}\right] \\
\quad \text { Post-condition }:\{\mathrm{a} \in \mathrm{X} \Rightarrow \mathrm{a} \in \mathrm{Y}\}
\end{gathered}
$$

Since OR-Set is RA-linearizable w.r.t. the specification in Example 3.4 (proved in Section 4.1), the possible values of $X$ and $Y$ can be computed by enumerating their RA-linearizations. The postcondition follows from the conjunction of the assertions in each replica. Let us consider the validation of the assertion of right hand side with the following RA-linearization:

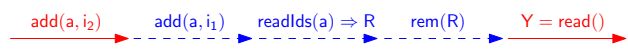

We have in red color and with solid arrows the operations of the right hand side replica, and in blue with dashed arrows the left ones. Let us consider the sub-sequence of the linearization that is visible to the last operation $(Y=\operatorname{read}())$. Since the first operation $\left(\operatorname{add}\left(a, i_{2}\right)\right)$ is issued on the same replica, it must be visible to it. Let us now consider different cases for the operations of the other replica that are visible to the read: (a) if the remove operation $\operatorname{rem}(\mathrm{R})$ is not visible to it, then the assertion is trivially true, because $\left(a, i_{2}\right)$ is in the resulting set according to the specification, and therefore the consequent of the assertion is valid. Assume from now on that rem $(R)$ is visible to it, there are two cases (b) if $\left(a, i_{2}\right)$ does not belong to $R$ the consequent of the assertion is valid, since the addition of $\left(a, i_{2}\right)$ is necessarily visible to the read operation, and we conclude as before, (c) on the other hand, if $\left(a, i_{2}\right) \in \mathrm{R}$ we have that the antecedent of the implication is falsified, and therefore the assertion is also valid.

Here we have considered only one RA-linearization, but it is not hard to see that commuting the operations of the different replicas renders the same argument. Importantly, this reasoning was done entirely at the level of the RA-linearizations (i.e. the specification) of the data type.

For the assertion on the left hand side replica, since visibility includes the order between operations issued on the same replica, we get that $\operatorname{add}\left(a, i_{1}\right)$ is ordered before readlds $(a) \Rightarrow R$ in every RAlinearization. Since add $\left(a, i_{1}\right)$ is also visible to readlds $(a) \Rightarrow R$, we get that $\left(a, i_{1}\right) \in R$. Similarly, every RA-linearization will order $\operatorname{rem}(\mathrm{R})$ before the $\operatorname{read}()$ on the left replica, which implies that if $a \in X$, then $\left(a, i_{2}\right) \notin R$. Assuming the contrary, i.e., $\left(a, i_{2}\right) \in R$, implies that $R=\left\{\left(a, i_{1}\right),\left(a, i_{2}\right)\right\}$ and since $\operatorname{rem}(R)$ is visible and linearized before $X=\operatorname{read}()$, we get that $a \notin X$.

\section{PROVING REPLICATION-AWARE LINEARIZABILITY}

We describe a methodology for proving that CRDT objects are RA-linearizable which relies on two properties: (1) the effectors of any two concurrent operations (i.e., not visible to each other) commute, which is inherent to CRDT objects, and (2) the existence of a refinement mapping [Abadi and Lamport 1991; Lynch and Vaandrager 1995] showing that each effector produced by an operation $\ell$, respectively each query $\ell$, is simulated by the execution of $\ell$ (or its counterpart through a queryupdate rewriting $\gamma$ ) in the specification Spec. This methodology is used in two forms depending on how the linearization is defined along an execution, which may affect the precise definition of the refinement mapping. We illustrate these two variations using OR-Set and RGA as examples. 


\subsection{Execution-Order Linearizations}

We first consider the case of CRDT objects, e.g., OR-Set, for which the order in which operations are executed at the origin replica defines a valid RA-linearization. We say that such objects admit execution-order linearizations. We start by formalizing the two properties we use to prove RAlinearizability.

Given a history $h=(\mathrm{L}, \mathrm{vis})$, we say that two operations $\ell_{1}$ and $\ell_{2}$ are concurrent, denoted $\ell_{1} \triangle_{\mathrm{vis}} \ell_{2}$, when $\left(\ell_{1}, \ell_{2}\right) \notin$ vis and $\left(\ell_{2}, \ell_{1}\right) \notin$ vis. In general, CRDTs implicitly require that the effectors of concurrent operations commute:

Commutativity: for every trace $\operatorname{tr}$ with $h(t r)=(\mathrm{L}$, vis $)$, and every two operations $\ell_{1}, \ell_{2} \in \mathrm{L}$, if $\ell_{1} \triangleleft_{\text {vis }} \ell_{2}$, then

$$
\forall \sigma \in \Sigma . \delta_{\ell_{1}}\left(\delta_{\ell_{2}}(\sigma)\right)=\delta_{\ell_{2}}\left(\delta_{\ell_{1}}(\sigma)\right)
$$

where $\delta_{\ell_{1}}$ and $\delta_{\ell_{2}}$ are the effectors of $\ell_{1}$ and resp., $\ell_{2}$.

Example 4.1. For OR-Set, two add, resp., remove, effectors commute because they both add, resp., remove, element-id pairs, while an add and a remove effector commute when they are concurrent because the element-id pairs removed by the remove effector are different from the pair added by the add effector (since the add is not visible to remove).

Commutativity implies that for every linearization lin of the operations in an execution, which is consistent with the visibility relation, every replica state $\sigma$ in that execution can be obtained by applying the delivered effectors in the order defined by lin (between the operations corresponding to those effectors). Indeed, by the causal delivery assumption, the order in which effectors are applied at a given replica is also consistent with visibility. Therefore, the only differences between the order in which effectors were applied to obtain $\sigma$ in that execution and the linearization order lin involve effectors of concurrent operations, which commute.

LEMMA 4.2. Let $\rho$ be an execution of an object o satisfying Commutativity, $h=(\mathrm{L}$, vis) the history of $\rho$, and ( $\mathrm{L}$, seq) a linearization of the operations in $\mathrm{L}$ (possibly, rewritten using a queryupdate rewriting $\gamma)$, consistent with vis. For each local configuration $\left(\mathrm{L}_{\mathrm{r}}, \sigma_{\mathrm{r}}\right)$ in $\rho$,

$$
\sigma_{\mathrm{r}}=\delta_{\ell_{n}}\left(\ldots\left(\delta_{\ell_{1}}\left(\sigma_{0}\right)\right) \ldots\right)
$$

where $\delta_{\ell}$ denotes the effector of operation $\ell, \sigma_{0}$ is the initial replica state, and seq $\downarrow_{L_{r}}=\ell_{1} \ldots \ell_{n}$.

In order to relate the CRDT object with its specification we use refinement mappings, which are "local" in the sense that they characterize the evolution of a single replica in isolation. A refinement mapping abs associates replica states with states of the specification, such that any update or query applied on a replica state $\sigma$ can be mimicked by the corresponding operation in the specification starting from abs $(\sigma)$. Moreover, the resulting states in the two steps must be again related by abs. Formally, given a query-update rewriting $\gamma$, we define Refinement as the existence of a mapping abs such that:

Simulating effectors: For every effector $\delta$ corresponding to a (query-)update operation $\ell$, and every state $\sigma \in \Sigma$,

$$
\sigma^{\prime}=\delta(\sigma) \Longrightarrow \operatorname{abs}(\sigma) \stackrel{\operatorname{upd}(\gamma(\ell))}{\longrightarrow} \operatorname{abs}\left(\sigma^{\prime}\right)
$$

where $\hookrightarrow$ is the transition function of Spec.

Simulating generators: For every query $\mathrm{m}$, and every $\sigma \in \Sigma$,

$$
\theta(\sigma, \mathrm{m}, a)=\left(b,{ }_{-},\right) \Longrightarrow \operatorname{abs}(\sigma) \stackrel{\ell}{\hookrightarrow} \operatorname{abs}(\sigma)
$$


where $\ell=\mathrm{m}(a) \Rightarrow b$. Recall that $\theta(\sigma, \mathrm{m}, a)$ stands for applying the generator of $\mathrm{m}$ with argument $a$ on the state $\sigma$. Also, for every query-update $\mathrm{m}$, and $\sigma \in \Sigma$,

$$
\theta(\sigma, \mathrm{m}, a)=\left(b,{ }_{-}\right) \Longrightarrow \operatorname{abs}(\sigma) \stackrel{\operatorname{qry}(\gamma(\ell))}{\longrightarrow} \operatorname{abs}(\sigma) .
$$

Example 4.3. Consider the OR-Set object, its specification in Example 3.4, and the query-update rewriting in Example 3.6. Also, let abs be a refinement mapping defined as the identity function. The effector of an $\operatorname{add}(a) \Rightarrow k$ operation, rewritten by $\gamma$ to $\operatorname{add}(a, k)$, and the $\operatorname{add}(a, k)$ operation of the specification have the same effect. Similarly, the effector of a query-update remove $(a) \Rightarrow R$ operation, rewritten by $\gamma$ to $(\operatorname{readIds}(a) \Rightarrow R$, remove $(a, R))$, and the remove $(a, R)$ operation of the specification have the same effect. Applying the query operation read() on a state $\sigma$ results in the same return value A as applying the same query in the context of the specification on the state $\operatorname{abs}(\sigma)=\sigma$. Finally, for the query-update $\operatorname{remove}(\mathrm{a}) \Rightarrow \mathrm{R}$, executing its generator in a state $\sigma$ results in the same return value $\mathrm{R}$ as executing the query readIds $(a) \Rightarrow R$ introduced by the query-update rewriting in the specification state $\operatorname{abs}(\sigma)=\sigma$.

Next, we show that any object o satisfying Commutativity and Refinement is RA-linearizable. Given a history $h=(\mathrm{L}, \mathrm{vis})$ of a trace $t r$, the execution-order linearization of $h$ is the sequence $\left(\gamma(\mathrm{L})\right.$, seq) such that $\gamma\left(\ell_{1}\right)$ occurs before $\gamma\left(\ell_{2}\right)$ in lin iff gen $\left(\ell_{1}\right)$ occurs before gen $\left(\ell_{2}\right)$ in $t r$, for every two labels $\ell_{1}, \ell_{2} \in \mathrm{L}$. An object o admits execution-order linearizations if for any history $h=(\mathrm{L}$, vis) of a trace $t r$, the execution-order linearization is an RA-linearization of $h$ w.r.t. Spec and $\gamma$.

THEOREM 4.4. Any object that satisfies Commutativity and Refinement admits execution-order linearizations.

PROOF. (Sketch) Clearly, any execution-order linearization is consistent with visibility. Then, we have to argue that queries can be explained by applying the updates visible to them in linearization order (item (iii) in Definition 3.5). More precisely, we have to show that for each query $\ell_{\mathrm{qr}}$, the sequence $\mathrm{seq}^{\prime} \cdot \ell_{\mathrm{qr}}$ where seq' is the projection of seq on the set of updates visible to $\ell_{\mathrm{qr}}$ is admitted by the specification. First, by Lemma 4.2, the state $\sigma$ of the replica where $\ell_{\mathrm{qr}}$ is applied is obtained by applying the effectors of the operations visible to $\ell_{\mathrm{qr}}$ in the linearization order. Then, by Refinement, every effector is simulated by the corresponding operation in the context of the specification. This implies that $\operatorname{abs}\left(\sigma_{0}\right) \stackrel{\text { seq }^{\prime}}{\longrightarrow}$ abs $(\sigma)$, where $\sigma_{0}$ is the initial replica state. The query $\ell_{\mathrm{qr}}$ is also simulated by the same operation in the context of the specification, which implies that abs $(\sigma) \stackrel{\ell_{\mathrm{qr}}}{\longrightarrow} \operatorname{abs}(\sigma)$. These two facts imply that $\operatorname{abs}\left(\sigma_{0}\right) \stackrel{\text { seq' }^{\prime} \cdot \ell_{\mathrm{qr}}}{\longrightarrow}$ abs $(\sigma)$ which means that $\mathrm{seq}^{\prime} \cdot \ell_{\mathrm{qr}}$ is admitted by the specification.

Finally, the projection of seq on the updates is admitted by the specification since (1) any trace $t r$ can be extended with a query operation $\ell$ that sees all the (query-)updates in $t r$, and (2) the validity of $\ell$ w.r.t. the specification (shown above) implies in particular that the sequence of updates is admitted by the specification.

We now discuss the issue of the non-deterministic specifications of Wooki, where an $\operatorname{addBetween}(a, b, c)$ operation inserts the element $b$ at a random position between $a$ and $c$ (when $a$ and $c$ are not adjacent). The specification of the query read is however deterministic returning the whole list stored in the state (excluding tombstone elements). Although the specification of these objects is non-deterministic, the proof of Lemma 4.2 and Refinement imply that the objects are convergent, in the sense that any two queries seeing the same set of updates return the same value. Indeed, by Lemma 4.2, the replica states where such queries are applied are the same (assuming that effectors are deterministic, which is the case in all the CRDTs we are aware of) and the existence of a refinement mapping implies

Proc. ACM Program. Lang., Vol. 1, No. CONF, Article 1. Publication date: January 2018. 


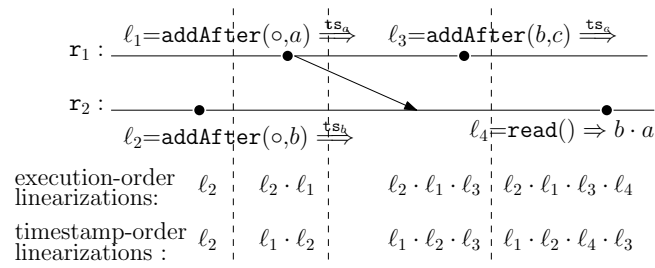

Fig. 8. Execution-order and timestamp-order linearizations for RGA. Here $t s_{a}<t s_{b}<t s_{c}$.

that the specification states corresponding to these replica states are also the same. The fact that the queries are deterministic concludes the proof of convergence.

\subsection{Timestamp-Order Linearizations}

CRDT objects such as RGA in Listing 1, that use timestamps for conflict resolution, may not admit execution-order linearizations. For instance, Fig. 8 shows an execution of RGA where two replicas $r_{1}$ and $r_{2}$ execute two addAfter invocations, and an addAfter invocation followed by a read invocation, respectively. An execution-order linearization which by definition, is consistent with the order in which the operations are applied at the origin replica, will order $\operatorname{addAfter}(\circ, b)$ before addAfter $(\circ, a)$. The result of applying these two operations in this order in the specification Spec(RGA) (defined in Example 3.3) is the list $a \cdot b$. However, if the timestamp $t s_{a}$ of $a$ is smaller than the timestamp $t s_{b}$ of $b$, a read that sees these two operations will return the list $b \cdot a$, which is different than the one obtained by applying the same operations in the context of Spec(RGA) in linearization order. Such a sequence is not a valid RA-linearization (w.r.t. Spec(RGA)). Therefore, we consider a variation of the proof methodology described in Sec. 4.1 where the linearizations are additionally consistent with the order of timestamps generated by the operations. We describe this instantiation using the RGA as an example (showing that it is RA-linearizable w.r.t. Spec(RGA)). More precisely, we consider linearizations where the operations that generate a timestamp, i.e., addAfter, are ordered in the same order as their timestamps. For instance, in the execution of Fig. 8, addAfter $(\circ, a)$ will be ordered before $\operatorname{addAfter}(\circ, b)$ because $t s_{a}$ is smaller than $t s_{b}$ (irrespective of the order between the generators). Moreover, to extend the notion of timestamp ordering to operations $\ell$ that don't generate timestamps, i.e., invocations of remove and read, we consider a "virtual" timestamp which is defined as the maximal timestamp of any operation visible to $\ell$ (or $\perp$ if no operation is visible to $\ell$ ), and require that the linearization is consistent with the order between both "real" 9 and "virtual" timestamps. For instance, the "virtual" timestamp of the read in Fig. 8 is $t s_{b}$ because it sees addAfter $(\circ, a)$ and $\operatorname{addAfter}(\circ, b)$. Then, a valid RA-linearization will order the read operation before the other addAfter (b, c) operation, since the timestamp $t s_{c}$ of the latter is bigger than the "virtual" timestamp $t s_{b}$ of the read. The operations that have the same timestamp (which is possible due to "virtual" timestamps) ${ }^{10}$ are ordered as they execute at the origin replica For instance, the read with "virtual" timestamp $t s_{b}$ is ordered after addAfter $(\circ, b)$ that has the same timestamp $t s_{b}$ since it executes later at the origin replica.

Formally, for a history $h=\left(\mathrm{L}\right.$, vis), we define the timestamp $\operatorname{ts}_{h}(\ell)$ of a label $\ell$ in the context of the history $h$ to be $\operatorname{ts}_{h}(\ell)=\operatorname{ts}(\ell)$ if $\operatorname{ts}(\ell) \neq \perp$ and $\operatorname{ts}_{h}(\ell)=\max \left\{\operatorname{ts}\left(\ell^{\prime}\right):\left(\ell^{\prime}, \ell\right) \in\right.$ vis $\}$, otherwise. Given a history $h=(\mathrm{L}, \mathrm{vis})$ of a trace $t r$, the timestamp-order linearization of $h$ is the sequence (L, seq) such that $\gamma\left(\ell_{1}\right)$ occurs before $\ell_{2}$ in lin iff $\operatorname{ts}_{h}\left(\ell_{1}\right)<\operatorname{ts}_{h}\left(\ell_{2}\right)$ or gen $\left(\ell_{1}\right)$ occurs before gen $\left(\ell_{2}\right)$ in $t r$, for every

\footnotetext{
${ }^{9}$ That is, timestamps generated by the operation itself.

${ }^{10}$ Among operations that have the same timestamp $t s$, there is exactly one operation generating $t s$, the rest of the operations have $t s$ as a "virtual" timestamp (i.e., they don't generate timestamps and the maximal timestamp they see is $t s$ ).
} 
two labels $\ell_{1}, \ell_{2} \in \mathrm{L}$. An object o admits timestamp-order linearizations if for any history $h=(\mathrm{L}$, vis) of a trace $t r$, the timestamp-order linearization is an RA-linearization of $h$ w.r.t. Spec. ${ }^{11}$

Proving admittance of timestamp-order linearizations relies on Commutativity and a slight variation of Refinement where intuitively, an effector generating a timestamp ts has to be simulated by a specification operation only when it is applied on a state $\sigma$ that doesn't "store" a greater timestamp than $t s$ (other effectors are treated as before). Formally, the set ts $(\sigma)$ of timestamps in a state $\sigma$ contains all the timestamps $t s$ generated by effectors applied to obtain $\sigma$. For RGA, the set of timestamps in a state $\sigma$ is the set of all timestamps stored in its timestamp tree. We define Refinement $t_{\mathrm{ts}}$ by modifying the "Simulating effectors" part of Refinement as follows:

Simulating effectors: For every effector $\delta$ of an operation $\ell$,

$$
\forall \sigma \in \Sigma . \operatorname{ts}(\ell) \nless \operatorname{ts}(\sigma) \wedge \sigma^{\prime}=\delta(\sigma) \Longrightarrow \operatorname{abs}(\sigma) \stackrel{\ell}{\hookrightarrow} \operatorname{abs}\left(\sigma^{\prime}\right)
$$

where $\operatorname{ts}(\ell) \nless \operatorname{ts}(\sigma)$ means that $\operatorname{ts}(\ell)$ is not smaller than any timestamp in $\operatorname{ts}(\sigma)$.

Example 4.5. Let us consider the RGA object, its specification in Example 3.3, and a refinement mapping abs which relates a replica state $(\mathrm{N}$, Tomb) with a specification state $(l, T)$ where the sequence $l$ is given by the function traverse in read queries when ignoring tombstones, i.e., $l=\operatorname{traverse}(\mathrm{N}, \emptyset)$, and $T=$ Tomb. It is obvious that remove effectors and read queries are simulated by the corresponding specification operations. Effectors of $\operatorname{addAfter}(a, b) \stackrel{t_{\mathrm{s}}}{\Rightarrow}$ operations are simulated by the specification operation $\operatorname{addAfter}(\mathrm{a}, \mathrm{b})$ only when $t s_{\mathrm{b}}$ is greater than all the timestamps stored in the replica state where it applies. Thus, let (N, Tomb) be a replica state such that $t s<t s_{\mathrm{b}}$ for every $t s$ with $\left({ }_{-}, t s,{ }_{-}\right) \in \mathrm{N}$. The result of applying the effector $\delta$ of $\operatorname{addAfter}(\mathrm{a}, \mathrm{b}) \stackrel{\mathrm{ts} \mathrm{b}}{\Rightarrow}$ is to add $b$ as a child of $a$. Then, applying traverse on the new state will result in a sequence where $b$ is placed just after a because it has the highest timestamp among the children of $a$. (and all the nodes in the tree $\mathrm{N}$ ). This corresponds exactly to the sequence obtained by applying the operation addAfter $(a, b)$ in the context of the specification.

The proof of an object o admitting timestamp-order linearizations if it satisfies Commutativity and Refinement $_{\mathrm{ts}}$ is similar to the one of Theorem 4.4. Intuitively, although Refinement $\mathrm{ts}_{\mathrm{ts}}$ is weaker than Refinement, the fact that the timestamp-order linearizations are consistent with the order between the timestamps generated by the operations, allows to show that any sequence of effectors consistent with such a linearization can be simulated by a sequence of specification operations.

THEOREM 4.6. Any object that satisfies Commutativity and Refinement ${ }_{\mathrm{ts}}$ admits timestamporder linearizations.

We remark that the API of a CRDT can impact on whether it is RA-linearizable. For instance, a slight variation of the RGA in Listing 1 with the same state, but with an interface with a method $\operatorname{addAt}(\mathrm{a}, \mathrm{k})$ to insert an element $\mathrm{a}$ at an index $\mathrm{k}$, introduced in [Attiya et al. 2016], would not be RA-linearizable w.r.t. an appropriate sequential specification (see [Enea et al. 2019]).

\section{COMPOSITIONALITY OF RA-LINEARIZABILITY}

We investigate the issue of whether the composition of a set of objects satisfying RA-linearizability is also RA-linearizable. While this is not true in general, we show that the composition of objects that admit execution-order or timestamp-order linearizations is RA-linearizable under the assumption that they share the same timestamp generator.

\footnotetext{
${ }^{11}$ For simplicity, we ignore query-update rewritings. The CRDTs with timestamp-order linearizations we investigated don't require such rewritings.
}

Proc. ACM Program. Lang., Vol. 1, No. CONF, Article 1. Publication date: January 2018. 


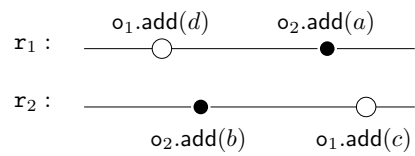

Fig. 9. A history of two OR-Sets. Each operation is visible only at the origin, so visibility is given by the horizontal lines.

\subsection{Object Compositions and RA-Linearizability}

Given two objects $\mathrm{o}_{1}$ and $\mathrm{o}_{2}$, the semantics of their composition $\mathrm{o}_{1} \otimes \mathrm{o}_{2}$ is the standard product of the LTSs corresponding to $\mathrm{o}_{1}$ and $\mathrm{o}_{2}$, respectively. Formally, given $\llbracket \mathrm{o}_{1} \rrbracket=\left(\mathrm{GC}_{1}, \mathbb{A}_{1}, \mathrm{gc}_{0}^{1}, \rightarrow_{1}\right)$ and $\llbracket \mathrm{o}_{2} \rrbracket=\left(\mathrm{GC}_{2}, \mathbb{A}_{2}, \mathrm{gc}_{0}^{2}, \rightarrow_{2}\right)$, we define $\llbracket \mathrm{o}_{1} \otimes \mathrm{o}_{2} \rrbracket=\left(\mathrm{GC}_{1} \times \mathrm{GC}_{2}, \mathbb{A}_{1} \cup \mathbb{A}_{2},\left(\mathrm{gc}_{0}^{1}, \mathrm{gc}_{0}^{2}, \emptyset\right), \rightarrow_{1,2}\right)$ where

$$
\begin{aligned}
\rightarrow_{1,2}= & \left\{\left(\left(\mathrm{gc}_{1}, \mathrm{gc}_{2}\right), a_{1},\left(\mathrm{gc}_{1}^{\prime}, \mathrm{gc}_{2}\right)\right):\left(\mathrm{gc}_{1}, a_{1}, \mathrm{gc}_{1}^{\prime}\right) \in \rightarrow_{1}\right\} \\
& \cup\left\{\left(\left(\mathrm{gc}_{1}, \mathrm{gc}_{2}\right), a_{2},\left(\mathrm{gc}_{1}, \mathrm{gc}_{2}^{\prime}\right)\right):\left(\mathrm{gc}_{2}, a_{2}, \mathrm{gc}_{2}^{\prime}\right) \in \rightarrow_{2}\right\}
\end{aligned}
$$

The history of a trace $\operatorname{tr}$ of $\mathrm{o}_{1} \otimes \mathrm{o}_{2}$ records a "global" visibility relation between the operations in the trace, i.e., which operations of $o_{1}$ or $o_{2}$ are visible when issuing an operation of $o_{1}$, and similarly, for operations of $\mathrm{o}_{2}$. Formally, $h(t r)=(\mathrm{L}$, vis $)$ where $\mathrm{L}$ is the set of labels occurring in $t r$, and $\left(\ell_{1}, \ell_{2}\right) \in$ vis if there exists a replica $r$ such that eff ${ }_{r}\left(\ell_{1}\right)$ occurs before gen ${ }_{r}\left(\ell_{2}\right)$ in the trace $t r$ In general, vis may not be a partial order since the causal delivery assumption holds only among operations of the same object. The set of histories $\mathbb{H}$ ist $\left(\mathrm{o}_{1} \otimes \mathrm{o}_{2}\right)$ of the composition $\mathrm{o}_{1} \otimes \mathrm{o}_{2}$ is the set of histories $h$ of a trace $\operatorname{tr}$ of $\mathrm{o}_{1} \otimes \mathrm{o}_{2}$.

For two specifications $\mathrm{Spec}_{1}$ and $\mathrm{Spec}_{2}$ of two objects $\mathrm{o}_{1}$ and $\mathrm{o}_{2}$, respectively, the composition $\mathrm{Spec}_{1} \otimes \mathrm{Spec}_{2}$ is the set of interleavings of sequences in $\mathrm{Spec}_{1}$ and $\mathrm{Spec}_{2}$, respectively. More precisely, $\mathrm{Spec}_{1} \otimes \mathrm{Spec}_{2}$ is the set of sequences $(\mathrm{L}, \mathrm{seq})$ such that their projection on labels of $\mathrm{o}_{1}$, resp., $\mathrm{o}_{2}$, is admitted by $\mathrm{Spec}_{1}$, resp., $\mathrm{Spec}_{2}$. We say that the composition $\mathrm{o}_{1} \otimes \mathrm{O}_{2}$ is RA-linearizable if every history of $\mathrm{o}_{1} \otimes \mathrm{O}_{2}$ is RA-linearizable w.r.t. $\mathrm{Spec}_{1} \otimes \mathrm{Spec}_{2}$. The extension to a set of objects is defined as usual.

Linearizability [Herlihy and Wing 1990] ensures that the composition of a set of linearizable objects is also linearizable. More precisely, it ensures that for every history, any per-object linearizations, concerning the operations of a single object, can be combined into a global linearization, concerning all the operations in the history. By combining linearizations, we mean constructing a global linearization whose projections on the operations of a single object are exactly the per-object linearizations considered in the beginning. However, this is not true for our notion of RA-linearizability. A counterexample is given in Fig. 9. The operations of $o_{1}$ are represented using blank circles and the operations of $\mathrm{o}_{2}$ using filled circles. The operations of $o_{1}$ can be linearized to $o_{1}$. add $(c) \cdot o_{1}$. add (d) (this is a valid RA-linearization since any sequence of add operations is admitted by Spec(OR-Set)) while the operations of $\mathrm{O}_{2}$ can be linearized to $\mathrm{O}_{2} \cdot \operatorname{add}(\mathrm{a}) \cdot \mathrm{O}_{2} \cdot \operatorname{add}(\mathrm{b})$. There is no RA-linearization of this history whose projections on each of the two objects correspond to these per-object linearizations. Trying to construct a linearization where $\mathrm{o}_{2}$. $\operatorname{add}(\mathrm{a})$ occurs before $\mathrm{O}_{2}$. add(b) will imply that $\mathrm{o}_{1}$.add(d) must occur before $\mathrm{o}_{1}$.add(c) (since it must be consistent with the visibility relation), which contradicts the linearization of $o_{1}$, and similarly for the other case, when trying to construct a global linearization consistent with the linearization of $\mathrm{o}_{2}$ 's operations. A reader knowledgeable of the literature on linearizability may notice that this discrepancy between standard linearizability and RA-linearizability comes from the fact that the partial order defining a history in the case of standard linearizability is actually an interval order $^{12}$, while in the case of RA-linearizability it is an arbitrary partial order.

\footnotetext{
${ }^{12}$ A partial order $R$ is an interval-order if $\{(a, b),(c, d)\} \subseteq R$ implies that $(a, d) \in R$ or $(c, b) \in R$, for every $a, b, c, d$.
} 


\subsection{Composition: Execution-Order Linearizability}

Although not all per-object RA-linearizations can be combined into global RA-linearizations, this may still be true in some cases. For the history in Fig. 9, the operations of $o_{1}$ can also be linearized to $\mathrm{o}_{1} \cdot \operatorname{add}(\mathrm{d}) \cdot \mathrm{o}_{1} \cdot \operatorname{add}(\mathrm{c})$ which enables a global linearization $\mathrm{o}_{1} \cdot \operatorname{add}(\mathrm{d}) \cdot \mathrm{o}_{2} \cdot \operatorname{add}(\mathrm{a}) \cdot \mathrm{o}_{2} \cdot \operatorname{add}(\mathrm{b}) \cdot \mathrm{o}_{1} \cdot \operatorname{add}(\mathrm{c})$ whose projection on each object is consistent with the per-object linearization (we take the same linearization $\mathrm{O}_{2}$. $\operatorname{add}(\mathrm{a}) \cdot \mathrm{O}_{2}$. $\operatorname{add}(\mathrm{b})$ for $\mathrm{O}_{2}$ ).

The following theorem shows that in the case of RA-linearizable objects that admit executionorder linearizations, there always exist per-object RA-linearizations that can be combined into global RA-linearizations, hence their composition is RA-linearizable and moreover, it also admits executionorder linearizations. A first preliminary result states that the order in which concurrent operations are executed at the origin replica can be permuted arbitrarily while still leading to a valid trace. More precisely, for every linearization lin of the visibility in the history of a trace, there exists another valid trace where operations are executed at the origin replica in the order defined by lin.

LEMMA 5.1. Let tr be a trace of an object $\mathrm{o}$ and $h(t r)=(\mathrm{L}$, vis). Then, for every sequence ( $\mathrm{L}, \mathrm{lin})$ which is consistent with vis (i.e., vis $\cup$ lin is acyclic), there exists a trace $\operatorname{tr}^{\prime}$ of o such that gen $\left(\ell_{1}\right)$ occurs before gen $\left(\ell_{2}\right)$ in tr $^{\prime}$ iff $\ell_{1}$ occurs before $\ell_{2}$ in lin. Moreover, $t^{\prime}$ has the same history as tr.

PROOF. (Sketch) We define a dependency relation $\odot$ between actions as follows: $\operatorname{gen}_{\mathrm{r}_{1}}\left(\ell_{1}\right) \odot$ eff $_{r_{2}}\left(\ell_{2}\right)$ iff $r_{1}=r_{2}$ or $\ell_{1}=\ell_{2}$, gen gen $_{r_{1}}\left(\ell_{1}\right) \odot \operatorname{gen}_{r_{2}}\left(\ell_{2}\right)$ iff $r_{1}=r_{2}$, eff $r_{r_{1}}\left(\ell_{1}\right) \odot$ gen $_{r_{2}}\left(\ell_{2}\right)$ iff $r_{1}=r_{2}$, and eff $_{r_{1}}\left(\ell_{1}\right) \odot$ eff $_{r_{2}}\left(\ell_{2}\right)$ iff $r_{1}=r_{2}$. Given a trace $t r=t r_{1} \cdot a_{1} \cdot a_{2} \cdot t r_{2}$, we say that a trace $\operatorname{tr}^{\prime}=\operatorname{tr}_{1} \cdot a_{2} \cdot a_{1} \cdot t_{2}$ is derived from $t r$ by a $\odot$-valid swap iff $a_{1}$ and $a_{2}$ are not related by $\odot$. Using standard reasoning about traces of a concurrent system, it can be shown that if $t r$ is a trace of $o$, then any trace $t r^{\prime}$ derived through a sequence of $\odot$-valid swaps is also a trace of o. Moreover, $t r^{\prime}$ has the same history as $t r$ Then, by the definition of the visibility relation vis in the history of a trace and the causal delivery assumption, it can be shown that given a trace tr of o containing two actions gen $\left(\ell_{1}\right)$ and gen $\left(\ell_{2}\right)$ such that $\ell_{1}$ and $\ell_{2}$ are concurrent and gen $\left(\ell_{1}\right)$ occurs before gen $\left(\ell_{2}\right)$, there exists another trace $t r^{\prime}$ of o that is defined through a sequence of $\odot$-valid swaps, where gen $\left(\ell_{2}\right)$ occurs before gen $\left(\ell_{1}\right)$. Thus, the order of concurrent generator actions can be permuted arbitrarily, concluding the lemma.

Lemma 5.1 implies that given an RA-linearizable object o, if it admits execution-order linearizations, then every linearization of a history of o consistent with visibility is a valid RA-linearization.

LEMMA 5.2. Let o be an object which is RA-linearizable w.r.t. a specification Spec and admits execution-order linearizations. Then, for every history $h=(\mathrm{L}, \mathrm{vis})$ of o and every sequence $(\mathrm{L}, \mathrm{lin})$ which is consistent with vis, we have that $(\mathrm{L}, \mathrm{lin})$ is an RA-linearization of $h$ w.r.t. Spec.

PROOF. (Skech) Let (L, lin) be a sequence consistent with the visibility relation of $h$. By Lemma 5.1, there exists a trace $\operatorname{tr}$ of o such that gen $\left(\ell_{1}\right)$ occurs before gen $\left(\ell_{2}\right)$ in $\operatorname{tr}$ iff $\ell_{1}$ occurs before $\ell_{2}$ in lin. The definition of execution-order linearizations implies that $(\mathrm{L}, \operatorname{lin})$ is an RA-linearization of $h(t r)=h$.

Lemma 5.2 is the essential ingredient for proving that the composition of a set of RA-linearizable objects that admit execution-order linearizations is also RA-linearizable. Intuitively, given a history $h$ with multiple such objects, any linearization of $h$ is a valid RA-linearization since each of its projections on the set of operations of a single object is a linearization of the per-object visibility relation ${ }^{13}$ and thus, a valid RA-linearization of that object.

THEOREM 5.3. The composition of a set of RA-linearizable objects that admit execution-order linearizations is RA-linearizable and admits execution-order linearizations.

\footnotetext{
${ }^{13}$ By definition, the visibility relation in the history of an object composition $o_{1} \otimes o_{2}$ projected on operations of $o_{1}$, resp., $o_{2}$, is exactly the visibility relation stored in the global configurations of $o_{1}$, resp., $o_{2}$ (at the end of the execution).
}

Proc. ACM Program. Lang., Vol. 1, No. CONF, Article 1. Publication date: January 2018. 


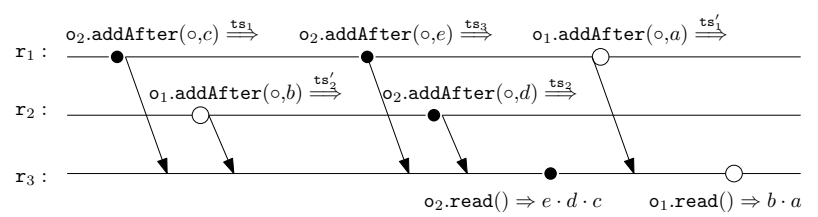

Fig. 10. A history in the composition $\otimes$ of two RGAs.

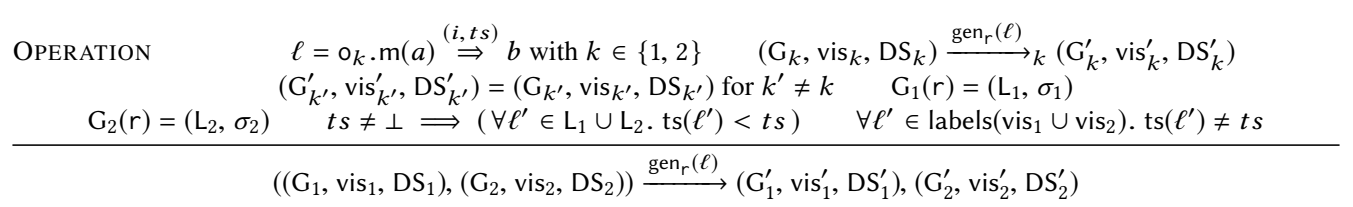

Fig. 11. The transition rule for generators in the object composition operator $\otimes_{\mathrm{ts}}$.

\subsection{Composition: Timestamp-Order Linearizability}

Theorem 5.3 does not apply to objects that admit timestamp-order linearizations. The "unrestricted" object composition $\otimes$ allows different objects to generate timestamps independently, and in "conflicting" orders along some execution. For instance, Fig. 10 shows a history with two RGA objects $\mathrm{o}_{1}$ and $\mathrm{o}_{2}$. We assume that $t s_{1}<t s_{2}<t s_{3}$ and $t s_{1}^{\prime}<t s_{2}^{\prime}$ (the order between other timestamps is not important). The operations of $o_{1}$, resp., $o_{2}$, can be linearized to

$$
\begin{aligned}
& \text { - } o_{1} \cdot \operatorname{addAfter}(o, a) \cdot o_{1} \cdot \operatorname{addAfter}(o, b) \cdot o_{1} \cdot \operatorname{read}() \Rightarrow b \cdot a \\
& -o_{2} \cdot \operatorname{addAfter}(o, c) \cdot o_{2} \cdot \operatorname{addAfter}(o, d) \cdot \\
& \mathrm{o}_{2} \cdot \operatorname{addAfter}(o, e) \cdot \mathrm{o}_{2} \cdot \operatorname{read}() \Rightarrow \mathrm{e} \cdot \mathrm{d} \cdot \mathrm{c}
\end{aligned}
$$

These are the only RA-linearizations possible. There is no "global" linearization consistent with these per-object linearizations: ordering addAfter $(\circ, a)$ before $\operatorname{addAfter}(\circ, b) \operatorname{implies}$ that $\operatorname{addAfter}(\circ, \mathrm{e})$ occurs before addAfter $(o, d)$ which contradicts the second linearization above. We solve this problem by constraining the composition operator $\otimes$ such that intuitively, all objects share a common timestamp generator. This ensures that each new timestamp is bigger than the timestamps used by operations delivered to a replica, independently of the object to which they pertain. For instance, the history of Fig. 10 would not be admitted because $t s_{1}^{\prime}$ should be bigger than $t s_{3}$ (since the operation that received $t s_{3}$ from the timestamp generator originates from the same replica as the operation receiving $t s_{1}^{\prime}$ at a later time) and $t s_{2}$ should be bigger than $t s_{2}^{\prime}$. These two constraints together with $t s_{1}^{\prime}<t s_{2}^{\prime}$ contradict $t s_{2}<t s_{3}$. While this requires a modification of the algorithms, where the timestamp generator is a parameter, this has no algorithmic or run-time cost, and in fact a similar idea have been suggested in the systems literature (e.g. [Enes et al. 2017]).

Using again Lemma 5.1, which ensures that the generators of "concurrent" operations can be executed in any order, we get that for any RA-linearizable object o which admits timestamp-order linearizations, every linearization of a history of o which is also consistent with the order between timestamps is a valid RA-linearization. For a history $h=(\mathrm{L}$, vis $)$, let $<_{h}$ be an order between the labels in $\mathrm{L}$ such that $\ell_{1}{ }_{h} \ell_{2}$ iff $\operatorname{ts}_{h}\left(\ell_{1}\right)<\mathrm{ts}_{h}\left(\ell_{2}\right)$.

LEMMA 5.4. Let $\mathrm{o}$ be an object which is RA-linearizable w.r.t. a specification Spec and admits timestamp-order linearizations. Then, for every history $h=(\mathrm{L}, \mathrm{vis})$ of $\mathrm{o}$ and every sequence $(\mathrm{L}, \mathrm{lin})$ which is consistent with vis and $<_{h}$, we have that $(\mathrm{L}, \mathrm{lin})$ is an RA-linearization of $h$ w.r.t. Spec.

We define a restriction $\otimes_{\mathrm{ts}}$ of the object composition $\otimes$ such that the set of histories $h=(\mathrm{L}$, vis) in the composition $\mathrm{o}_{1} \otimes_{\mathrm{ts}} \mathrm{O}_{2}$ satisfy the property that the order between timestamps (of all objects) is consistent with the visibility relation vis (i.e., vis $U \prec_{h}$ is acyclic). With respect to the "unrestricted" composition $\otimes$ defined in Sec. 5.1, we only modify the transition rule corresponding to generators, 
as shown in Fig. 11. This ensures that a new generated timestamp is bigger than all the timestamps "visible" to the replica executing that generator (irrespectively of the object). Its extension to a set of objects is defined as usual. The composition operator $\otimes_{\mathrm{ts}}$ is called shared timestamp generator composition. Practically, if we were to consider the standard timestamp mechanism used in CRDTs, i.e., each replica maintains a counter which is increased monotonically with every new operation (originating at the replica or delivered from another replica) and timestamps are defined as pairs of replica identifiers and counter values, then $\otimes_{\mathrm{ts}}$ can be implemented using a "shared" counter which increases monotonically with every new operation, independently of the object on which it is applied.

The following theorem shows that the composition of RA-linearizable objects that admit executionorder or timestamp-order linearizations is RA-linearizable, provided that all the objects share the same timestamp generator.

THEOREM 5.5. The shared timestamp generator composition of a set of RA-linearizable objects that admit execution-order or timestamp-order linearizations is $R A$-linearizable.

The theorem above shows that any shared timestamp generator composition of the objects mentioned in next section is RA-linearizable.

\section{MECHANIZING RA-LINEARIZABILITY PROOFS}

To validate our approach, we considered a range of CRDTs listed in Fig. 12 and mechanized their RA-linearizability proofs using Boogie [Barnett et al. 2005], a verification tool. More precisely, we mechanized the proofs of conditions like Commutativity and Refinement which imply RAlinearizability by the results in Section 4. Beyond operation-based CRDTs (discussed in the paper), we have also considered state-based CRDTs, where an update occurs only at the origin, and replicas exchange their states instead of operations, and states from other replicas are merged at the replica receiving them. The merge function corresponds to the least upper bound operator in a certain join semi-lattice defined over replica states.

For operation-based CRDTs, we have mechanized the proof of a strenghtening of Commutativity that avoids reasoning about traces and the proof of Refinement (or Refinement $t_{\mathrm{ts}}$ ). Concerning Commutativity, our proofs encode two effectors as a single procedure which executes on two equal copies of the replica state. In some cases, the precondition of this procedure encodes conditions which are satisfied anytime the two effectors are concurrent, e.g., the effector of an add and resp., remove of OR-Set are concurrent when the argument $\mathrm{k}$ of add is not in the argument $\mathrm{R}$ of remove. At least for the CRDTs we consider, such characterizations are obvious and apply generically to any conflict-resolution policy based on unique identifiers. In some cases, the effectors commute even if they are not concurrent, so no additional precondition is needed. We prove that the resulting states are identical after performing the effectors in different order in each of the states. Refinement (or Refinement $\mathrm{ts}_{\mathrm{s}}$ ) is reduced to proving that the refinement mapping is an inductive invariant for a lock-step execution of the CRDT implementation and its specification.

For state-based CRDTs, we have identified a set of conditions similar to those of operation-based CRDTs that imply RA-linearizability (see [Enea et al. 2019]). In this case, we don't rely on the causal delivery assumption. Extending their semantics with an auxiliary variable maintaining a correspondence between replica states and sets of operations that produced them, we extract the visibility relation between operations as in the case of operation-based CRDTs. This enables a similar reasoning about RA-linearizability. In particular, Commutativity is replaced by few conditions that now characterize the relationship between applying operations at a given replica and the merge function. 


\begin{tabular}{|l|c|c|}
\hline CRDT & Imp. & Lin. \\
\hline \hline Counter [Shapiro et al. 2011] & OB & EO \\
\hline PN-Counter [Shapiro et al. 2011] & SB & EO \\
\hline LWW-Register [Johnson and Thomas 1975] & OB & TO \\
\hline Multi-Value Reg. [DeCandia et al. 2007] & SB & EO \\
\hline LWW-Element Set [Shapiro et al. 2011] & SB & TO \\
\hline
\end{tabular}

\begin{tabular}{|l|c|r|}
\hline CRDT & Imp. & Lin. \\
\hline \hline 2P-Set [Shapiro et al. 2011] & SB & EO \\
\hline OR-Set [Shapiro et al. 2011] & OB & EO \\
\hline RGA [Roh et al. 2011] & OB & TO \\
\hline Wooki [Weiss et al. 2007] & OB & EO \\
\hline
\end{tabular}

Fig. 12. CRDTs proved RA-linearizable and the class of linearizations used. SB: State-Based, OB: Operation-Based, EO: Execution-Order, TO: Timestamp-Order.

\section{RELATED WORK}

Correctness Criteria. Burckhardt et al. [2014] gives the first formal framework where CRDTs and other weakly consistent replicated systems can be specified. Their CRDT specifications are defined in terms of sets of partial orders as opposed to our sequential specifications, which we think are easier to reason about when verifying clients. Beyond simpler specifications, RA-linearizability is related to their formalization of causal consistency, called causal convergence in [Bouajjani et al. 2017]. Overall RA-linearizability differs from causal convergence in three points: (1) query-update rewritings, which enable sequential specifications and avoid partial orders, (2) the linearization projected on updates must be admitted by the specification (intuitively, this ensures that the "final" convergence state is valid w.r.t. the specification), and (3) the linearization is required to be consistent with the visibility order from the execution, and not an arbitrary one as in causal convergence. The latter difference makes causal convergence not compositional.

Regarding convergence, RA-linearizability implies that there is a unique total order of updates, and therefore if at some point all updates are visible to all replicas, all subsequent query operations at any replica will return the same value. This is observably equivalent to strong eventual consistency [Gomes et al. 2017; Shapiro et al. 2011; Zeller et al. 2014]. RA-linearizability is also stronger than the session guarantees of Terry et al. [1994], but weaker than sequential consistency [Lamport 1979] and linearizability [Herlihy and Wing 1990]. RA-linearizable objects that admit executionorder linearizations are close to being linearizable since the operations are linearized as they were issued at the origin replica, relative to wall-clock time. This is similar to linearizability, where each operation appears to take effect instantaneously between the wall-clock time of its invocation its response. Unlike linearizability, RA-linearizability allows queries to return a response consistent with only a subsequence of its linearized-before operations.

Sequential Specifications for CRDTs. Perrin et al. [2014] provides Update Consistency (UC), a criterion which to the best of our knowledge is the first to consider sequential specifications and characterize linear histories of operations. However UC is not compositional due to an existential quantification over visibility relations like in causal convergence. Moreover, Perrin et al. [2014] doesn't investigate UC proof methodologies.

Jagadeesan and Riely [2018] provide a correctness criterion called SEC, which differs from RA-linearizability in several points: (1) Firstly, RA-linearizability has a global total order for updates, unlike SEC whose definition is quite complex. (2) Secondly, CRDT specifications in SEC are parameterized by a dependency relation at the level of the type's API. Then, SEC assumes that all independent operations commute and disregards their order even when issued by the same client. It is unclear how such a specification could adequately capture systems enforcing session guarantees [Terry et al. 1994]. RA-linearizability strives to preserve the guarantees of the underlying system network since certain operations that appear independent at the API level could be made data or control dependent by the client. This happens in our query-update rewritings where queries provide arguments for the subsequent update. (3) While SEC is also compositional, since operations from different objects are assumed independent, a history of two different SEC objects is trivially

Proc. ACM Program. Lang., Vol. 1, No. CONF, Article 1. Publication date: January 2018. 
SEC since the order between operations of different objects is ignored. We find this notion of composition problematic since the composition of specifications cannot capture causality between different objects, a common pattern when writing distributed applications (e.g. for referential integrity in a key-value store). In RA-linearizability the composition of a set of objects respects the client's causality as illustrated by the failure to combine some per-object linearizations in Fig. 9. ${ }^{14}$

Verification of CRDTs There are several works that approach the problem of verifying that a CRDT implementation is correct w.r.t. a specification. In [Attiya et al. 2016; Burckhardt 2014; Burckhardt et al. 2014] along with the formal specification, proofs of correctness of implementations are given for several CRDTs. Our Refinement property is inspired by the Replication Aware Simulations in [Burckhardt et al. 2014]. Compared to these simulations, our proofs remain at a much more abstract level - and are therefore simpler - since our specifications are simpler. We acknowledge however that their method is more general than ours which only applies to CRDTs satisfying RA-linearizability. Zeller et al. [2014] and Gomes et al. [2017] provide frameworks for the verification of CRDTs in Isabelle/HOL. Both works introduce a methodology to specify and prove CRDTs correct. Their proofs are similar to the simulations of [Burckhardt et al. 2014], albeit in a different specification language also based on partial orders.

\section{CONCLUSION}

We presented RA-linearizability, a correctness criterion inspired by linearizability, intended to simplify the specification of CRDTs by resorting to sequential reasoning for the specifications. We provide proof methodologies for RA-linearizability for some well documented CRDTs, and we prove that under certain conditions these proofs guarantee the compositionality of RA-linearizability. In the extended version of this paper [Enea et al. 2019] we show how our techniques extend to state-based CRDTs.

There are some limitations of RA-linearizability. Firstly, as we showed before, some CRDTs might not be RA-linearizable under a certain API, but a slight change in the API renders them RA-linearizable. We would like to investigate what constitutes an API that enables RA-linearizability specifications. secondly, while we argue that RA-linearizability simplifies specifications, we leave as future work to show whether it can be effectively used to verify client applications of a CRDT.

\section{REFERENCES}

[n. d.]. https://github.com/menesro/RA-linearizability-proofs

Martín Abadi and Leslie Lamport. 1991. The Existence of Refinement Mappings. Theor. Comput. Sci. 82, 2 (1991), $253-284$. https://doi.org/10.1016/0304-3975(91)90224-P

Hagit Attiya, Sebastian Burckhardt, Alexey Gotsman, Adam Morrison, Hongseok Yang, and Marek Zawirski. 2016. Specification and Complexity of Collaborative Text Editing. In Proceedings of the 2016 ACM Symposium on Principles of Distributed Computing, PODC 2016, Chicago, IL, USA, July 25-28, 2016. 259-268. https://doi.org/10.1145/2933057.2933090

Michael Barnett, Bor-Yuh Evan Chang, Robert DeLine, Bart Jacobs, and K. Rustan M. Leino. 2005. Boogie: A Modular Reusable Verifier for Object-Oriented Programs. In Formal Methods for Components and Objects, 4th International Symposium, FMCO 2005, Amsterdam, The Netherlands, November 1-4, 2005, Revised Lectures. 364-387. https: //doi.org/10.1007/11804192_17

Ahmed Bouajjani, Constantin Enea, Rachid Guerraoui, and Jad Hamza. 2017. On verifying causal consistency. In Proceedings of the 44th ACM SIGPLAN Symposium on Principles of Programming Languages, POPL 2017, Paris, France, January 18 20, 2017, Giuseppe Castagna and Andrew D. Gordon (Eds.). ACM, 626-638. http://dl.acm.org/citation.cfm?id=3009888

Sebastian Burckhardt. 2014. Principles of Eventual Consistency. Foundations and Trends in Programming Languages 1, 1-2 (2014), 1-150. https://doi.org/10.1561/2500000011

Sebastian Burckhardt, Alexey Gotsman, Hongseok Yang, and Marek Zawirski. 2014. Replicated data types: specification, verification, optimality. In The 41st Annual ACM SIGPLAN-SIGACT Symposium on Principles of Programming Languages, POPL '14, San Diego, CA, USA, January 20-21, 2014. 271-284. https://doi.org/10.1145/2535838.2535848

${ }^{14}$ There are however per-object linearizations for this history which can be merged into a global linearization (see Sec. 5). 
Giuseppe DeCandia, Deniz Hastorun, Madan Jampani, Gunavardhan Kakulapati, Avinash Lakshman, Alex Pilchin, Swaminathan Sivasubramanian, Peter Vosshall, and Werner Vogels. 2007. Dynamo: amazon's highly available key-value store. In Proceedings of the 21st ACM Symposium on Operating Systems Principles 2007, SOSP 2007, Stevenson, Washington, USA, October 14-17, 2007, Thomas C. Bressoud and M. Frans Kaashoek (Eds.). ACM, 205-220. https://doi.org/10.1145/1294261.1294281

Constantin Enea, Suha Orhun Mutluergil, Gustavo Petri, and Chao Wang. 2019. Replication-Aware Linearizability. (2019).

Vitor Enes, Paulo Sérgio Almeida, and Carlos Baquero. 2017. The Single-Writer Principle in CRDT Composition. In Proceedings of the Programming Models and Languages for Distributed Computing (PMLDC '17). ACM, New York, NY, USA, Article 4, 3 pages. https://doi.org/10.1145/3166089.3168733

Seth Gilbert and Nancy A. Lynch. 2002. Brewer's conjecture and the feasibility of consistent, available, partition-tolerant web services. SIGACT News 33, 2 (2002), 51-59. https://doi.org/10.1145/564585.564601

Victor B. F. Gomes, Martin Kleppmann, Dominic P. Mulligan, and Alastair R. Beresford. 2017. Verifying strong eventual consistency in distributed systems. PACMPL 1, OOPSLA (2017), 109:1-109:28. https://doi.org/10.1145/3133933

Maurice Herlihy and Jeannette M. Wing. 1990. Linearizability: A Correctness Condition for Concurrent Objects. ACM Trans. Program. Lang. Syst. 12, 3 (1990), 463-492. https://doi.org/10.1145/78969.78972

Radha Jagadeesan and James Riely. 2018. Eventual Consistency for CRDTs. In Programming Languages and Systems 27th European Symposium on Programming, ESOP 2018, Held as Part of the European Joint Conferences on Theory and Practice of Software, ETAPS 2018, Thessaloniki, Greece, April 14-20, 2018, Proceedings. 968-995. https://doi.org/10. 1007/978-3-319-89884-1_34

Paul R. Johnson and Robert Thomas. 1975. Maintenance of duplicate databases. RFC 677 (1975), 1-10. https://doi.org/10. 17487/RFC0677

Leslie Lamport. 1979. How to Make a Multiprocessor Computer That Correctly Executes Multiprocess Programs. IEEE Trans. Computers 28, 9 (1979), 690-691. https://doi.org/10.1109/TC.1979.1675439

Nancy A. Lynch and Frits W. Vaandrager. 1995. Forward and Backward Simulations: I. Untimed Systems. Inf. Comput. 121, 2 (1995), 214-233. https://doi.org/10.1006/inco.1995.1134

Mahsa Najafzadeh, Alexey Gotsman, Hongseok Yang, Carla Ferreira, and Marc Shapiro. 2016. The CISE tool: proving weakly-consistent applications correct. In Proceedings of the 2nd Workshop on the Principles and Practice of Consistency for Distributed Data,PaPoC@EuroSys 2016, London, United Kingdom, April 18, 2016. 2:1-2:3. https://doi.org/10.1145/ 2911151.2911160

Gérald Oster, Pascal Urso, Pascal Molli, and Abdessamad Imine. 2006. Data consistency for P2P collaborative editing. In Proceedings of the 2006 ACM Conference on Computer Supported Cooperative Work, CSCW 2006, Banff, Alberta, Canada, November 4-8, 2006, Pamela J. Hinds and David Martin (Eds.). ACM, 259-268. https://doi.org/10.1145/1180875.1180916

Matthieu Perrin, Achour Mostéfaoui, and Claude Jard. 2014. Update Consistency in Partitionable Systems. In Distributed Computing - 28th International Symposium, DISC 2014, Austin, TX, USA, October 12-15, 2014. Proceedings. 546-549. http://link.springer.com/content/pdf/bbm\%3A978-3-662-45174-8\%2F1.pdf

Hyun-Gul Roh, Myeongjae Jeon, Jinsoo Kim, and Joonwon Lee. 2011. Replicated abstract data types: Building blocks for collaborative applications. J. Parallel Distrib. Comput. 71, 3 (2011), 354-368. https://doi.org/10.1016/j.jpdc.2010.12.006

Marc Shapiro, Nuno Preguiça, Carlos Baquero, and Marek Zawirski. 2011. A comprehensive study of Convergent and Commutative Replicated Data Types. Research Report RR-7506. Inria - Centre Paris-Rocquencourt ; INRIA. 50 pages. https://hal.inria.fr/inria-00555588

Douglas B. Terry, Alan J. Demers, Karin Petersen, Mike Spreitzer, Marvin Theimer, and Brent B. Welch. 1994. Session Guarantees for Weakly Consistent Replicated Data. In Proceedings of the Third International Conference on Parallel and Distributed Information Systems (PDIS 94), Austin, Texas, USA, September 28-30, 1994. IEEE Computer Society, 140-149. https://doi.org/10.1109/PDIS.1994.331722

Stéphane Weiss, Pascal Urso, and Pascal Molli. 2007. Wooki: A P2P Wiki-Based Collaborative Writing Tool. In Web Information Systems Engineering - WISE 2007, 8th International Conference on Web Information Systems Engineering, Nancy, France, December 3-7, 2007, Proceedings (Lecture Notes in Computer Science), Boualem Benatallah, Fabio Casati, Dimitrios Georgakopoulos, Claudio Bartolini, Wasim Sadiq, and Claude Godart (Eds.), Vol. 4831. Springer, 503-512. https://doi.org/10.1007/978-3-540-76993-4_42

Peter Zeller, Annette Bieniusa, and Arnd Poetzsch-Heffter. 2014. Formal Specification and Verification of CRDTs. In Formal Techniques for Distributed Objects, Components, and Systems - 34th IFIP WG 6.1 International Conference, FORTE 2014. Held as Part of the 9th International Federated Conference on Distributed Computing Techniques, DisCoTec 2014, Berlin, Germany, June 3-5, 2014. Proceedings. 33-48. https://doi.org/10.1007/978-3-662-43613-4_3 


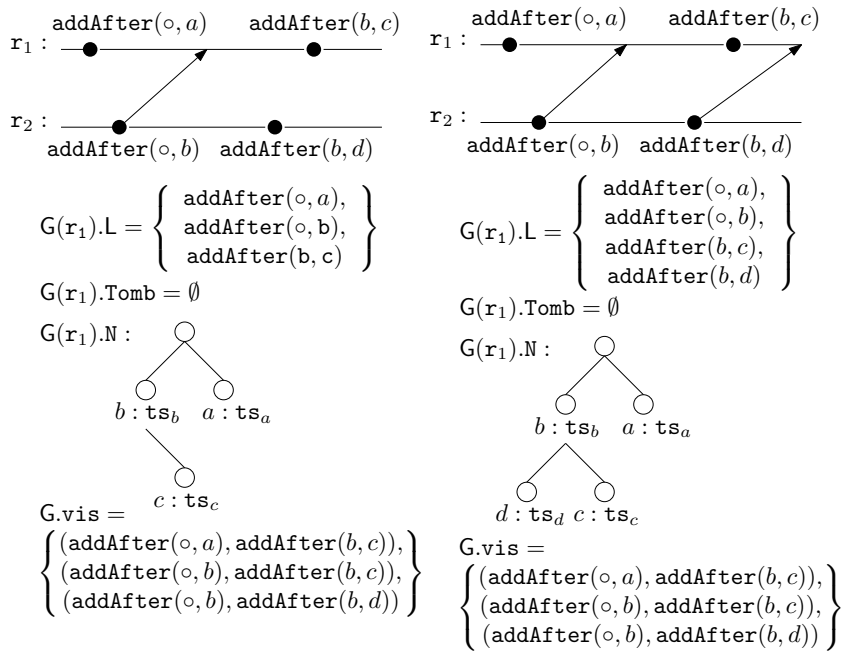

(a) (b)

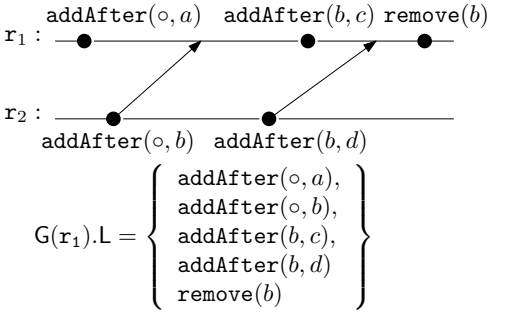

$\mathrm{G}\left(\mathrm{r}_{1}\right) \cdot \mathrm{Tomb}=\{b\}$

$\mathrm{G}\left(\mathrm{r}_{1}\right) \cdot \mathrm{N}$ :

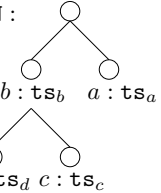

$d: \operatorname{ts}_{d} c: \mathrm{ts}_{c}$

G.vis $=$

$\left\{\begin{array}{l}(\text { addAfter }(o, a), \text { addAfter }(b, c)), \\ (\text { addAfter }(o, b), \operatorname{addAfter}(b, c)), \\ (\text { addAfter }(o, b), \operatorname{addAfter}(b, d)), \\ (\text { addAfter }(o, a), \operatorname{remove}(b)), \\ (\text { addAfter }(b, c), \operatorname{remove}(b)), \\ (\text { addAfter }(o, a), \operatorname{remove}(b)), \\ (\text { addAfter }(b, d), \operatorname{remove}(b))\end{array}\right\}$

(c)

Fig. 13. Example of the semantics of RGA.

\section{A TRANSITION RULES OF THE CRDT SEMANTICS}

Fig. 13 shows how some components of the semantics progress according to the rules of Fig. 7 for the RGA data type. In particular we shown: the local labels of replica $r_{1}\left(G\left(r_{1}\right) . L\right)$; its state, where we remove the $\sigma$ for succinctness (then $\mathrm{G}\left(\mathrm{r}_{1}\right) \cdot \sigma . \mathrm{N}$ becomes $\left.\mathrm{G}\left(\mathrm{r}_{1}\right) . \mathrm{N}\right)$; and the global visibility relation G.vis. The transition from Fig. 13b to Fig. 13c shows an transition where the operation remove(b) is executed by replica $r_{1}$. Notice in particular how the global visibility relation is extended.

In Fig. 13, the transition from Fig. 13a to Fig. 13b corresponds to a transition which extends the visibility of the operation addAfter $(b, d)$ to $r_{1}$. Notice that this is reflected in the $G\left(r_{1}\right)$.L component. The relation G.vis does not change since this relation only changes when a new operation is executed at the source replica.

\section{B IMPLEMENTATIONS OF OPERATION-BASED CRDT AND THEIR SEQUENTIAL SPECIFICATIONS}

\section{B.1 Operation-Based Counter and Its Sequential Specification}

Implementation: The operation-based counter of [Shapiro et al. 2011] is shown in Listing 3. It implements a counter interface with operations: inc(), dec() and read. A payload is a integer ctr. inc increase the counter value of replica state by 1 , dec decrease the counter value of replica state by 1 , and read returns the counter value of replica state.

Sequential Specification $\operatorname{Spec}($ Counter): Each abstract state $\phi$ is a integer. The sequential specification Spec(Counter) of counter is defined by: 


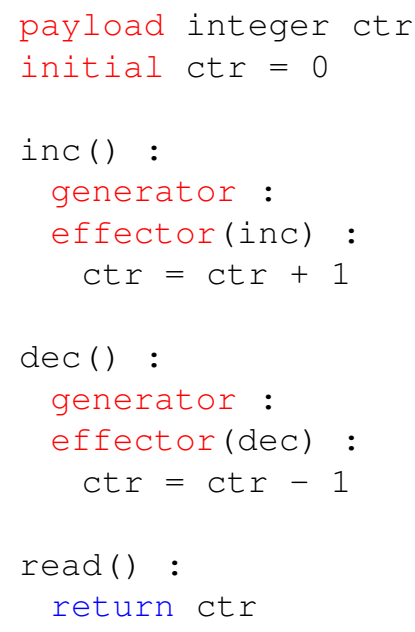

Listing 3. Pseudo-code of operation-based counter

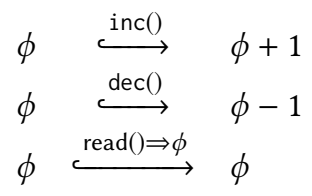

Method inc() increase the counter value by 1 . Method $\operatorname{dec}()$ decrease the counter value by 1 . Method $\operatorname{read}() \Rightarrow \mathrm{k}$ returns the counter value.

\section{B.2 Operation-Based Last-Writer-Win Register and its Sequential Specification}

Implementation: The operation-based last-writer-win Register (LWW-Register) of [Shapiro et al. 2011] is shown in Listing 4. It implements a register interface with operations: write $(a)$ and read. A payload is a tuple $(x, t s)$ of a data value $x$ and its timestamp $t s$. Here $x_{0}$ is an initial data and $t s_{0}$ is an initial timestamp.

write $(a)$ generates a new timestamp $t s^{\prime}$ and modifies the replica state into $\left(a, t s^{\prime}\right)$, and its effector uses the argument $\left(a, t s^{\prime}\right)$. When applying an effector with arguments $\left(a, t s^{\prime}\right)$ from a replica $\left(b, t s_{b}\right)$, the resulting replica state is $\left(a, t s^{\prime}\right)$ if $t s_{b}<t s^{\prime}$, and is $\left(b, t s_{b}\right)$ otherwise. read returns the data value of replica state.

Sequential Specification $\operatorname{Spec}(\mathbf{R e g})$ : Each abstract state $\phi$ is a data value. The sequential specification $\operatorname{Spec}(\mathrm{Reg})$ of LWW-register is defined by:

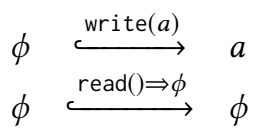

Method write(a) update the abstract state into data value $a$. Method $\operatorname{read}() \Rightarrow \phi$ returns the value of the register. 


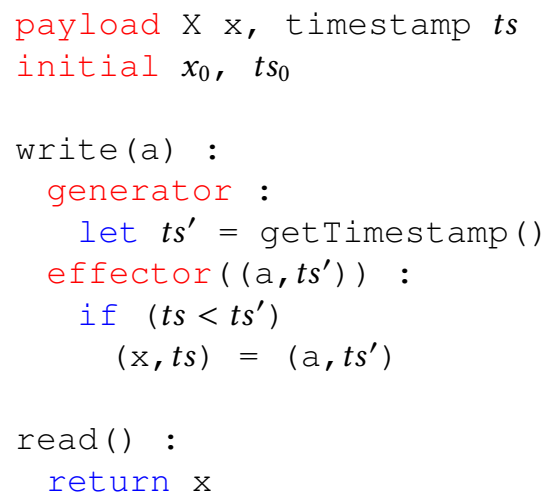

Listing 4. Pseudo-code of operation-based LWW-register

\section{B.3 Operation-Based Wooki and its Sequential Specification}

Implementation: The Wooki implementation of [Weiss et al. 2007] is given in Listing 5. Wooki is an optimized version of Woot [Oster et al. 2006]. To make our introduction more clear, we borrow the notion of W-character and W-string from Woot.

Wooki implements a list interface with operations: $\operatorname{addBetween}(a, b, c)$, remove $(a)$ and read. A

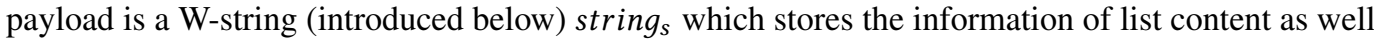
as tombstone.

A W-character $w$ is a tuple ( $i d, v$, degree, flag), and is used to stroe the information of an element of list. Here $i d$ is the identifier of $w$; $v$ is the value of $w$; degree is the degree of $w$; flag $\in\{$ true, false $\}$ is the flag of $w$ and indicates whether $w$ is "visible" in list. A identifier $i d$ of W-character is a unique timestamp. We use degree $(w)$ to denote the degree of $w$. A degree is a integer that is fixed when its $\mathrm{W}$-character is generated; when inserting an W-character into string $_{s}$, the degree of W-characters of string $_{s}$ influenced the position where this $\mathrm{W}$-character will be inserted into.

Let $\circ_{\text {begin }}$ and $\circ_{\text {end }}$ be two special values. Let $w_{\text {begin }}=\left({ }_{-}, \circ_{\text {begin }}, 0\right.$, true $)$ and $w_{\text {end }}=\left({ }_{-}, \circ_{\text {end }}\right.$, true $)$ be two special W-characters. A W-string is an ordered sequence of $\mathrm{W}$-characters $w_{\text {begin }} \cdot w_{1} \cdot \ldots$. $w_{n} \cdot w_{\text {end }}$. We never remove $\circ_{\text {begin }}$ or $\circ_{\text {end }}$, and never put value before $\circ_{\text {begin }}$ or after $\circ_{\text {end }}$. Since $w_{\text {begin }}$ and $w_{\text {end }}$ is fixed to be the head and tail of a W-string, in the latter part of this paper, when we considering the content of a W-string, we ignore $w_{\text {begin }}$ and $w_{\text {end }}$. We define the following functions for a W-string str:

- $|s t r|$ returns the length of str,

- $\operatorname{str}[p]$ returns the W-character at position $p$ in $s t r$. Here we assume that the first element of $s t r$ is at position 0 .

- $\operatorname{pos}(s t r, w)$ returns the position of W-character $w$ in str.

- insert $(s t r, w, p)$ inserts $\mathrm{W}$-character $w$ into str at position $p$.

- subseq $\left(s t r, w_{1}, w_{2}\right)$ returns the part of $s t r$ between the W-characters $w_{1}$ and $w_{2}$ (excluding $w_{1}$ and $\left.w_{2}\right)$.

- contains $(s t r, a)$ returns true if there exists a W-character in str with value $a$.

- values $(s t r)$ returns the sequence of visible (with true flag) values of str.

- getWchar $(s t r, a)$ returns the W-character with value $a$ in str.

- changeFlag(str,pos, $f$ ) changes the flag of $\operatorname{str}[$ pos] into boolean value $f$. 


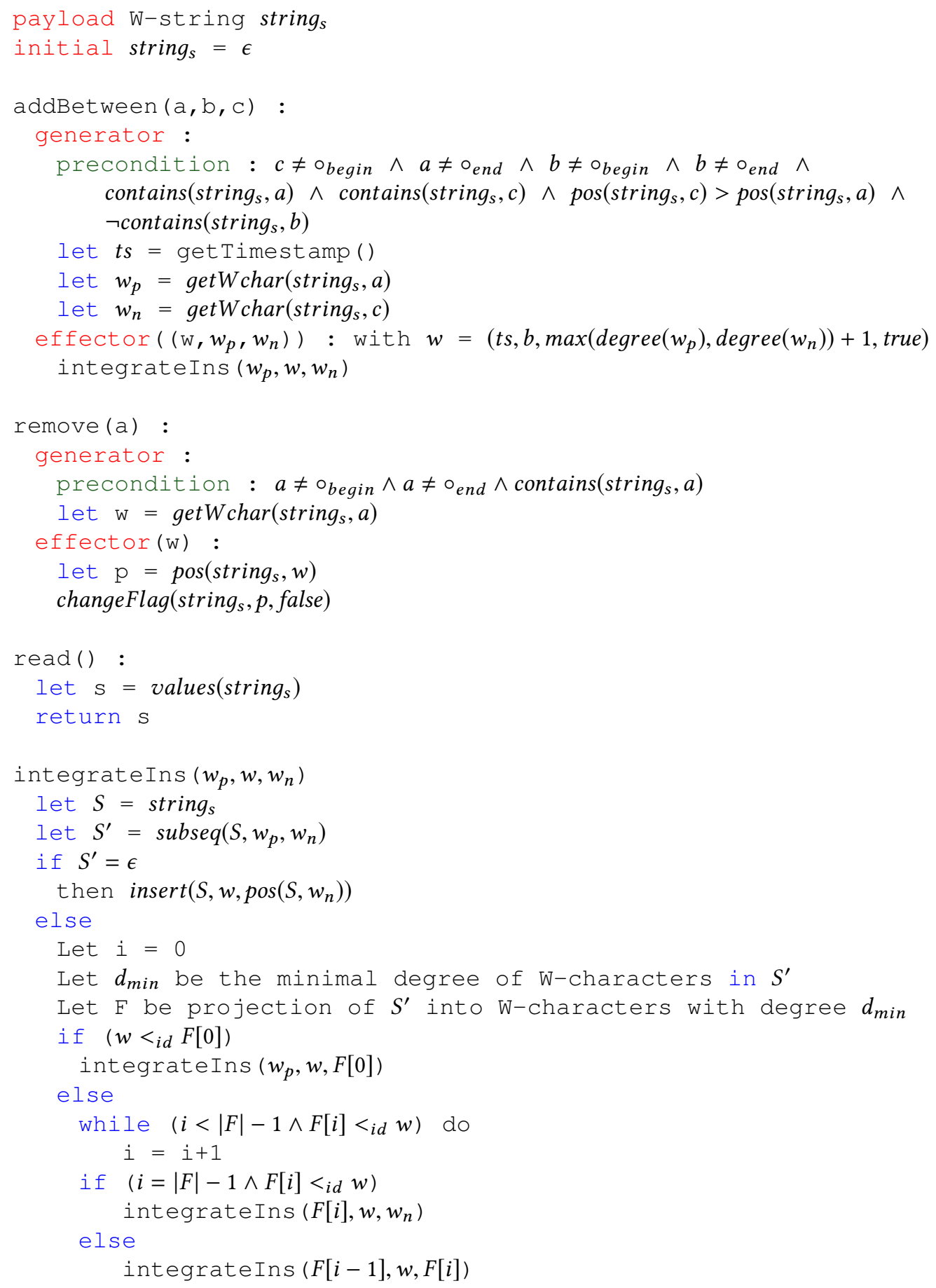

Listing 5. Pseudo-code of Wooki 
A total order $<_{i d}$ is given for identifiers of W-characters for conflict resolution, and let $<_{i d}$ be the total order of timestamps (identifiers of W-charcaters). Given a W-string str and two W-characters $w_{1}, w_{2}$ of str, we write $w_{1}<_{s t r} w_{2}$ to indicate that pos $\left(s t r, w_{1}\right)<\operatorname{pos}\left(s t r, w_{2}\right)$.

Note that, here we use addBetween method, which "adds a value between two values", and is different from the addAfter method of RGA. Also, note that a value can be inserted into the list only once.

addBetween $(a, b, c)$ intends to generate a new $\mathrm{W}$-character $w$ with value $b$ and put it between W-characters with values $a$ and $c$,respectively. $\operatorname{addBetween}(a, b, c)$ work as follows: First, we check the precondition, such as three exists W-characters with values $a$ and $c$ in string $_{s}$, and the $\mathrm{W}$-character with value $a$ is before the $\mathrm{W}$-character with value $c$ in string $g_{s}$. Then, let $w_{p}$

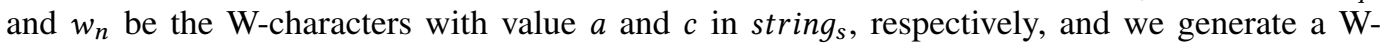
character $w=\left(t s, b, \max \left(\operatorname{degree}\left(w_{p}\right)\right.\right.$, degree $\left.\left(w_{n}\right)\right)+1$, true $)$ with value $b$. Finally, we call method integrateIns $\left(w_{p}, w, w_{n}\right)$ to put $w$ into string $g_{s}$ at some position between $w_{p}$ and $w_{n}$.

integrateIns $\left(w_{p}, w, w_{n}\right)$ is a recursive method and works as follows: If there are no W-character between $w_{p}$ and $w_{n}$, then $w$ is put after $w_{p}$. Else, Wooki selects a sequence $F$ of W-characters, such that each W-character of $F$ is between $w_{p}$ and $w_{n}$, and has minimal degree. Then, we choose the position of $w$ according to $<_{i d}$ order, and recursive call integrateIns $\left(w_{x}, w, w_{y}\right)$ for some $w_{x}, w_{y} \in$ $F \cup\left\{w_{p}, w_{n}\right\}$. We can see that, the minimal degree of W-characters of $\operatorname{subse} q\left(S, w_{x}, w_{y}\right)$ is larger than that of $\operatorname{subseq}\left(S, w_{p}, w_{n}\right)$, and $\operatorname{subseq}\left(S, w_{x}, w_{y}\right)$ is a sub-sequence of $\operatorname{subseq}\left(S, w_{p}, w_{n}\right)$.

remove(a) first finds the $\mathrm{W}$-character with value $a$ in string s $_{s}$ and then sets its flag into false. read uses values( string $_{s}$ ) to return the list content of string $_{s}$.

Sequential Specification $\operatorname{Spec}($ Wooki): Each abstract state $\phi=(l, T)$ contains a sequence $l$ of elements of a given type and a set $T$ of elements appearing in the list. The element $l$ is the list of all input values, whether already removed or not; while $T$ stores the removed values and is used as tombstone. The sequential specification Spec(Wooki) of list with add-between interface is defined by:

$$
\begin{aligned}
& \left(\left(l_{1} \cdot a \cdot l_{2} \cdot l_{3} \cdot c \cdot l_{4}, T\right) \mid b \text { is fresh, } a \neq \circ_{\text {end }}, c \neq \circ_{\text {begin }}\right) \\
& \left((l, T) \mid a \in l, a \neq o_{\text {begin }}, a \neq o_{\text {end }}\right) \\
& \begin{array}{ll}
\stackrel{\operatorname{rddBetween}(a, b, c)}{\stackrel{\operatorname{remove}(a)}{\longrightarrow}} & \left(l_{1} \cdot a \cdot l_{2} \cdot b \cdot l_{3} \cdot c \cdot l_{4}, T\right) \\
\stackrel{\operatorname{read}() \Rightarrow(l / T)}{\longrightarrow} & (l, T \cup\{a\}) \\
& (l, T)
\end{array}
\end{aligned}
$$

The method addBetween $(a, b, c)$ puts $b$ at some random position between $a$ and $c$ in $l$, assuming that each value is put into list at most once. Method remove $(a)$ adds $a$ into $T$, hence removing $a$ from the list for subsequent calls to the read method. Finally, $\operatorname{read}() \Rightarrow s$ returns the list content excluding any element appearing in $T$. Assume that the initial value of list is $\left(\circ_{\text {begin }} \cdot \circ_{\text {end }}, \emptyset\right)$, we never put value after $\circ_{\text {end }}$ or before $\circ_{\text {begin }}$, and $\circ_{\text {begin }}$ and $\circ_{\text {end }}$ are never removed. We will sometimes ignore the value $\circ_{\text {begin }}$ and $\circ_{\text {end }}$ from the result of read.

\section{DISCUSSION ABOUT LIST WITH INTERFACE OF INDEX (ADDAT)}

In this section, we consider list specification with addAt method, which puts a value at an index instead of putting a value after another value or between two values.

We first give two version of list specifications with addAt method, while one of them does not use tombstone and one of them uses tombstone. We prove that RGA is not RA-linearizable w.r.t any of them.

Then, we give a third version of list specification with addAt method, Spec(addAt3), where we use a "local version of index", and each method is required to returns the "local list content". We prove that RGA is RA-linearizable w.r.t Spec(addAt3). 


\section{C.1 A first version of list with addAt Interface}

The first version of list with addAt interface is as follows: A list uses the following three methods:

- $\operatorname{addAt}(\mathrm{a}, \mathrm{k})$ : Inserts value $a$ into position $k$ of the list of replica state. For $k$ exceeding the list size of replica state, $k$ will be inserted at the end of the list.

- remove(a): Remove value $a$ from list of the replica state.

$-\operatorname{read}() \Rightarrow \mathrm{s}$ : Returns the list content of the replica state.

Here we assume the first element of a sequence is at position 0 .

Our RGA algorithm of Sec.2 can be modified as follows for this interface: To $\operatorname{do} \operatorname{addAt}(a, k)$, we work as follows:

- Let $s=\operatorname{traverse}(N, T o m b)$ be the list content in replica state. If $s=\epsilon$, then let $b=\circ$; else, if $|s| \geq k$, then let $b=s[k-1]$; else, let $b=s[|s|-1]$. Here let $|s|$ be the length of $s$.

- Work as addAfter $(b, a)$. Especially, the effector of $\operatorname{addAt}(a, k)$ is the effector of $\operatorname{addAfter}(b$, a).

\section{C.2 Two Sequential Specification for list with addAt Interface}

Depending on whether use tombstone or not, there are two sequential specification for list with addAt interface.

Sequential specification $\operatorname{Spec}($ addAt1): The first sequential specification Spec(addAt1) of list with addAt interface does not use tombstone. Each abstract state $\phi=l$ contains a sequence $l$ of elements of a given type. The element $l$ is the list of values that has been input and not removed yet. The sequential specification Spec(addAt1) of list with addAt interface is defined by:

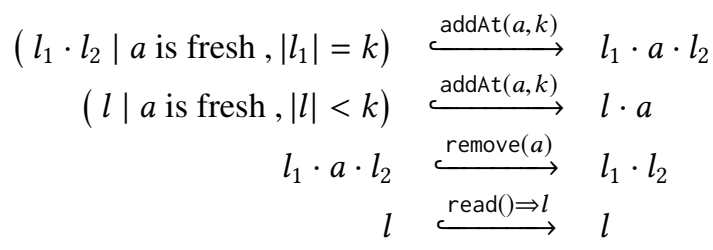

If $\left|l_{1} \cdot l_{2}\right| \geq k$ and $\left|l_{1}\right|=k$, the method addAt $(a, k)$ puts $a$ immediately after $l_{1}$, assuming that each value is put into list at most once; Else, if $|l|<k$, the method addAt $(a, k)$ puts $a$ immediately after $l$, assuming that each value is put into list at most once. Method remove $(a)$ remove $a$ from $l_{1} \cdot a \cdot l_{2}$. $\operatorname{read}() \Rightarrow l$ returns the list content.

Sequential specification $\operatorname{Spec}(\operatorname{addAt} 2)$ : The second sequential specification Spec(addAt2) of list with addAt interface uses tombstone. Each abstract state $\phi=(l, T)$ contains a sequence $l$ of elements of a given type and a set $T$ of elements appearing in the list. The element $l$ is the list of all input values, whether already removed or not; while $T$ stores the removed values and is used as tombstone. We can safely assume that $l$ contains more or equal values than $T$. The sequential specification Spec(addAt2) of list with addAt interface is defined by:

$$
\begin{array}{rll}
\left(\left(l_{1} \cdot l_{2}, T\right) \mid a \text { is fresh },\left|l_{1} / T\right|=k\right) & \stackrel{\operatorname{addAt}(a, k)}{\longrightarrow} & \left(l_{1} \cdot a \cdot l_{2}, T\right) \\
((l, T) \mid a \text { is fresh },|l / T|<k) & \stackrel{\operatorname{addAt}(a, k)}{\longrightarrow} & (l \cdot a, T) \\
((l, T) \mid a \text { occurs in } l) & \stackrel{\operatorname{remove}(a)}{\longrightarrow} & (l, T \cup\{a\}) \\
(l, T) & \stackrel{\operatorname{read}() \Rightarrow(l / T)}{\longrightarrow} & (l, T)
\end{array}
$$

If $l_{1} \cdot l_{2} \geq k$ and $\left|l_{1} / T\right|=k$, the method $\operatorname{addAt}(a, k)$ puts $a$ immediately after $l_{1}$, assuming that each value is put into list at most once; Else, if $|l / T|<k$, the method addAt $(a, k)$ puts $a$ immediately 
after $l$, assuming that each value is put into list at most once. Method remove( $a$ ) adds $a$ into $T$, hence removing $a$ from the list for subsequent calls to the read method. Finally, $\operatorname{read}() \Rightarrow s$ returns the list content excluding any element appearing in $T$.

Note that the addAt method of Spec(addAt2) is nondeterministic, since the values of $T$ in $l_{1}$ does not influence exeuction. For example, $(a \cdot b,\{a\}) \stackrel{\text { addAt }(c, 0)}{\longleftrightarrow}(c \cdot a \cdot b,\{a\})$ and $(a \cdot b,\{a\}) \stackrel{\text { addAt }(c, 0)}{\longleftrightarrow}$ $(a \cdot c \cdot b,\{a\})$ are both valid transitions. However, in the proof of Lemma C.1 we prove that when we consider only executions where each value can be removed only once, the admitted sequences of Spec(addAt2) is a subset of the admitted sequences of Spec(addAt1). Or we can say, the behavior of Spec(addAt2) is "deterministic"to some extent.

\section{C.3 RGA not RA-linearizable w.r.t Spec(addAt1) or Spec(addAt2)}

Fig. 14 shows an example that is a history of RGA and is not RA-linearizable w.r.t Spec(addAt1) or Spec(addAt2). Here we assume that $t s_{a}<t s_{b}<t s_{c}<t s_{d}<t s_{e}$. We also draw the replica state of replica $r_{2}$ and $r_{3}$ after the execution of $h$ in Fig. 14.

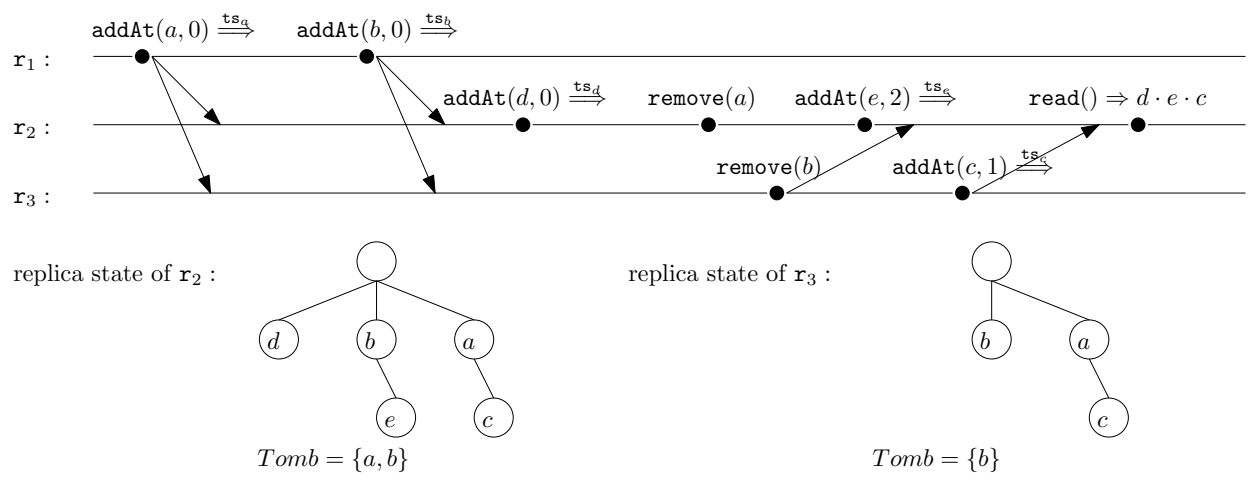

Fig. 14. An example history shows that RGA is not RA-linearizable w.r.t .

The following lemma states that RGA is not RA-linearizable w.r.t Spec(addAt1) or Spec(addAt2).

LEMMA C.1. RGA is not RA-linearizable w.r.t Spec(addAt1) or Spec(addAt2).

PROOF. Let $h$ be the history of Fig. 14. It is obvious that $h$ is a history of RGA. We prove that RGA is not RA-linearizable w.r.t $\operatorname{Spec}(\operatorname{addAt} 1)$ by proving that $h$ is not RA-linearizable w.r.t Spec(addAt1), and we prove this by showing that all possible linarizations of $h$ can not validate the $\operatorname{read}() \Rightarrow d \cdot e \cdot c$ operation. We list all possible linearization and their transitions in Spec(addAt1) as follows:

$$
\begin{aligned}
& -\epsilon \stackrel{\operatorname{addAt}(a, 0)}{\longrightarrow} a \stackrel{\operatorname{addAt}(b, 0)}{\longrightarrow} b \cdot a \stackrel{\text { remove }(b)}{\longrightarrow} a \stackrel{\operatorname{addAt}(c, 1)}{\longrightarrow} a \cdot c \stackrel{\operatorname{addAt}(d, 0)}{\longrightarrow} d \cdot a \cdot c \stackrel{\text { remove }(a)}{\longrightarrow} d \cdot c \stackrel{\operatorname{addAt}(e, 2)}{\longrightarrow} \\
& d \cdot c \cdot e \stackrel{\operatorname{read}() \Rightarrow d \cdot c \cdot e}{\stackrel{(a)}{\longrightarrow}} d \cdot e .
\end{aligned}
$$

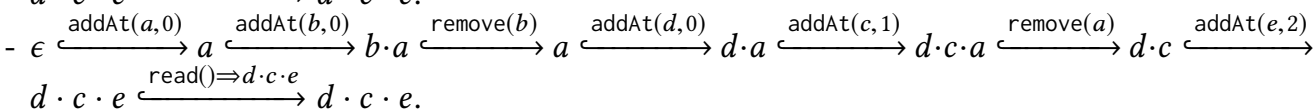

$$
\begin{aligned}
& \begin{aligned}
-\epsilon \stackrel{\operatorname{addAt}(a, 0)}{\longrightarrow} a \stackrel{\operatorname{addAt}(b, 0)}{\longrightarrow} b \cdot a \stackrel{\text { remove }(b)}{\longrightarrow} a \stackrel{\operatorname{addAt}(d, 0)}{\longrightarrow} d \cdot a \stackrel{\operatorname{read}() \Rightarrow d \cdot c \cdot e}{\longrightarrow} d \cdot c \cdot e . \\
d \cdot c \cdot e \stackrel{\text { remove }(a)}{\longrightarrow} d \stackrel{\operatorname{addAt}(c, 1)}{\longrightarrow} d \cdot c \stackrel{\operatorname{addAt}(e, 2)}{\longrightarrow}
\end{aligned} \\
& -\epsilon \stackrel{\operatorname{addAt}(a, 0)}{\longrightarrow} a \stackrel{\operatorname{addAt}(b, 0)}{\longrightarrow} b \cdot a \stackrel{\text { remove }(b)}{\longrightarrow} a \stackrel{\operatorname{addAt}(d, 0)}{\longrightarrow} d \cdot a \stackrel{\text { remove }(a)}{\longrightarrow} d \stackrel{\operatorname{addAt}(e, 2)}{\longrightarrow} d \cdot e \stackrel{\operatorname{addAt}(c, 1)}{\longrightarrow}
\end{aligned}
$$

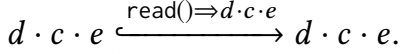

$$
\begin{aligned}
& \begin{aligned}
-\epsilon \underset{\operatorname{addAt}(e, 2)}{\longrightarrow} d \cdot c \cdot e \stackrel{\operatorname{addAt}(a, 0)}{\longrightarrow} d \stackrel{\operatorname{addAt}(b, 0)}{\longrightarrow} b \cdot a \stackrel{a d() \Rightarrow d \cdot c \cdot e}{\longrightarrow} d \cdot c \cdot e .
\end{aligned}
\end{aligned}
$$




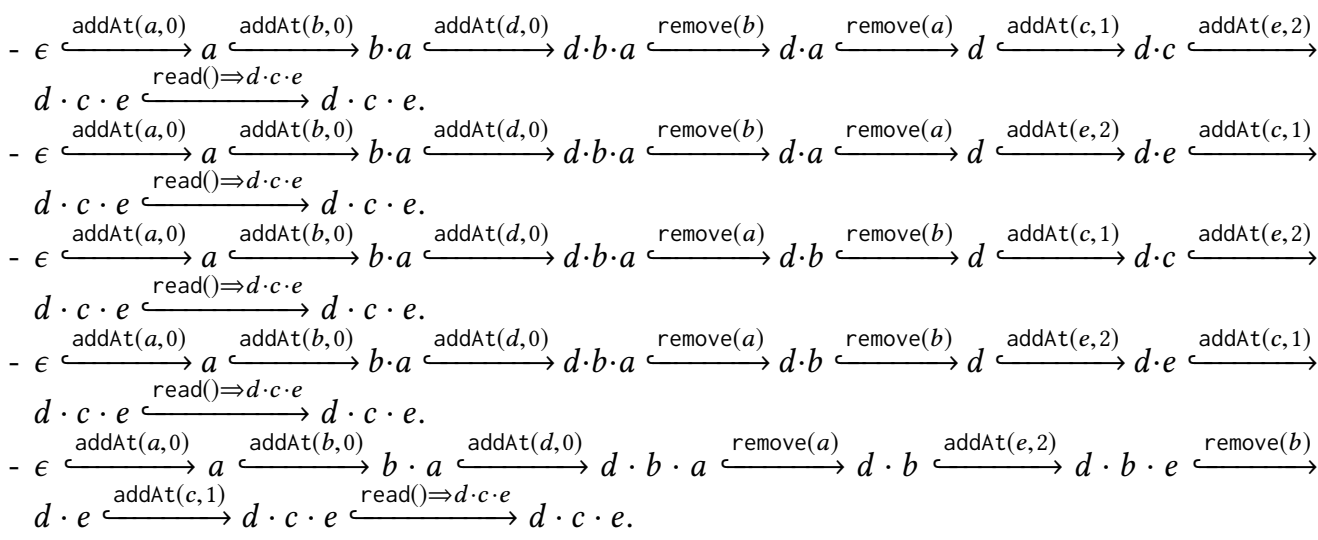

Since the last read operation returns $d \cdot c \cdot e$ instead of $d \cdot e \cdot c$, these linearization are not correct. Therefore, RGA is not RA-linearizable w.r.t Spec(addAt1).

We prove that RGA is not RA-linearizable w.r.t Spec(addAt2) by proving that, when we consider only executions where each value can be removed only once, the admitted sequences of Spec(addAt 2 ) is a subset of the admitted sequences of $\operatorname{Spec}(\operatorname{addAt} 1)$. We need to prove that there exits a relation $R$ between abstracts of Spec(addAt2) and Spec(addAt1), such that when there are transitions $\phi_{0} \stackrel{\ell_{0}}{\hookrightarrow}$ $\phi_{1} \ldots \stackrel{\ell_{n}}{\hookrightarrow} \phi_{n}$ of Spec(addAt2), and in $\ell_{0} \cdot \ldots \cdot \ell_{n}$ each value is removed at most once, we have that, (1) $\left(\phi_{0}, \phi_{0}^{\prime}\right) \in R$, where $\phi_{0}$ and $\phi_{0}^{\prime}$ is the initial abstract state of Spec(addAt2) and Spec(addAt1), respectively, and (2) if $\phi_{i} \stackrel{\ell_{i}}{\hookrightarrow} \phi_{i+1}$ is a transition of $\operatorname{Spec}(\operatorname{addAt} 2)$ and $\left(\phi_{i}, \phi_{i}^{\prime}\right) \in R$, then, $\phi_{i}^{\prime} \stackrel{\ell_{i}}{\hookrightarrow} \phi_{i+1}^{\prime}$ is in Spec(addAt1) and $\left(\phi_{i+1}, \phi_{i+1}^{\prime}\right) \in R$ for some abstract state $\phi_{i+1}^{\prime}$ of Spec(addAt1).

Let relation $R$ be defined as follows: $\left((l, T), l^{\prime}\right) \in R$, if $l^{\prime}=l / T$. Then, let us consider all possible transitions:

- If $\left(l_{1} \cdot l_{2}, T\right) \stackrel{\operatorname{addAt}(a, k)}{\longrightarrow}\left(l_{1} \cdot a \cdot l_{2}, T\right)$ is in Spec(addAt2) and $\left|l_{1} / T\right|=k$ : Then, we can see that $l^{\prime}=l / T=\left(l_{1} / T\right) \cdot\left(l_{2} / T\right)$, and then, $\left(l_{1} / T\right) \cdot\left(l_{2} / T\right) \stackrel{\operatorname{addAt}(a, k)}{\longrightarrow}\left(l_{1} / T\right) \cdot a \cdot\left(l_{2} / T\right)$ is in Spec(addAt1). Since $a$ is fresh in $l_{1} \cdot l_{2}$ and $l_{1} \cdot l_{2}$ contains more or equal values than $T$, we can see that $a \notin T$ and $\left(l_{1} / T\right) \cdot a \cdot\left(l_{2} / T\right)=\left(l_{1} \cdot a \cdot l_{2}\right) / T$. Or we can say, $\left(\left(l_{1} \cdot a \cdot l_{2}, T\right),\left(l_{1} / T\right) \cdot a \cdot\left(l_{2} / T\right)\right) \in R$.

- If $(l, T) \stackrel{\operatorname{addAt}(a, k)}{\longrightarrow}(l \cdot a, T)$ is in Spec(addAt2) and $|l / T|<k$ : Then, we can see that $l / T \stackrel{\operatorname{addAt}(a, k)}{\longrightarrow}(l / T) \cdot a$ is in $\operatorname{Spec}(\operatorname{addAt} 1)$ and $a$ is fresh in $l$. Since $l$ contains more or equal values than $T$, we can see that $a \notin T$. Then, $(l / T) \cdot a=(l \cdot a) / T$. Or we can say, $((l \cdot a, T),(l / T) \cdot a) \in R$.

- If $\left(l_{1} \cdot a \cdot l_{2}, T\right) \stackrel{\text { remove }(a)}{\longrightarrow}\left(l_{1} \cdot a \cdot l_{2}, T \cup\{a\}\right)$ is in Spec(addAt2): Since each value is removed only once, we can see that $a \notin T$. Then, $\left(\left(l_{1} \cdot a \cdot l_{2}, T\right),\left(l_{1} / T\right) \cdot a \cdot\left(l_{2} / T\right)\right) \in R .\left(l_{1} / T\right) \cdot a \cdot\left(l_{2} / T\right) \stackrel{\operatorname{remove}(a)}{\longrightarrow}$ $\left(l_{1} / T\right) \cdot\left(l_{2} / T\right)$ is in Spec(addAt1). Then, $\left(l_{1} / T\right) \cdot a \cdot\left(l_{2} / T\right)=\left(l_{1} \cdot a \cdot l_{2}\right) / T$. Or we can say, $\left(\left(l_{1} \cdot a \cdot l_{2}, T\right),\left(l_{1} / T\right) \cdot a \cdot\left(l_{2} / T\right)\right) \in R$.

- If $(l, T) \stackrel{\operatorname{read}() \Rightarrow(l / T)}{\longrightarrow}(l, T)$ is in Spec(addAt2): Obviously, $l / T \stackrel{\operatorname{read}() \Rightarrow(l / T)}{\longrightarrow} l / T$ is in Spec(addAt1) and $((l, T),(l / T)) \in R$.

Therefore, $R$ holds as required. Then, we can see that for each transitions $\phi_{0} \stackrel{\ell_{0}}{\hookrightarrow} \phi_{1} \ldots \stackrel{\ell_{n}}{\hookrightarrow} \phi_{n}$ of Spec(addAt2) where each value is removed at most once in $\ell_{0} \cdot \ldots \cdot \ell_{n}$, there exists transitions $\phi_{0}^{\prime} \stackrel{\ell_{0}}{\hookrightarrow} \phi_{1}^{\prime} \ldots \stackrel{\ell_{n}}{\longrightarrow} \phi_{n}^{\prime}$ of Spec(addAt1). Or we can say, for each sequence $\ell_{0} \cdot \ldots \cdot \ell_{n}$ where each value is removed at most once, $\ell_{0} \cdot \ldots \cdot \ell_{n} \in \operatorname{Spec}(\operatorname{addAt} 2)$ implies that $\ell_{0} \cdot \ldots \cdot \ell_{n} \in \operatorname{Spec}(\operatorname{addAt} 1)$. 
Since in the history $h$ of Fig. 14, each value is removed at most once, we can see that the set of possible linearization of $h$ of Spec(addAt2) is a subset of possible linearization of $h$ of Spec(addAt1). Since $h$ is not RA-linearizable w.r.t Spec(addAt1), we can see that $h$ is not RA-linearizable w.r.t Spec(addAt2). This completes the proof of this lemma.

\section{C.4 A second version of list with addAt Interface}

As the second version of list with addAt interface, let us introduce the interface of [Attiya et al. 2016] as below:

- $\operatorname{addAt}(\mathrm{a}, \mathrm{k}) \Rightarrow \mathrm{s}$ : Inserts value $a$ into position $k$ of list of the replica state, and then returns the updated list content of the replica state. For $k$ exceeding the list size of replica state, $a$ will be inserted at the end of the list.

- remove $(a) \Rightarrow \mathrm{s}$ : Remove value $a$ from list of the replica state, and returns the updated list content of the replica state.

$-\operatorname{read}() \Rightarrow \mathrm{s}$ : Returns the list content of the replica state.

Similarly, we assume the first element of a sequence is at position 0 .

Our RGA algorithm of Sec. 2 can be modified as follows for this interface:

- For addAt: Given argument $a$ and $k$ of addAt. Let $s=$ traverse $(N, T o m b)$ be the list content in replica state. If $s=\epsilon$, then let $b=0$; else, if $|s| \geq k$, then let $b=s[k-1]$; else, let $b=s[|s|-1]$.

Then, we work as addAfter(b,a). Especially, the effector of addAt(a, $k)$ is the effector of $\operatorname{addAfter}(b, a)$. After the effector of $\operatorname{addAfter}(b, a)$ is applied in the replica where this operation originates, let $s^{\prime}$ be the list content of replica state, and we return $s^{\prime}$.

- For remove: Given argument $a$, we work as remove(a). After the effector of remove(a) is applied in the replica where this operation originates, let $s^{\prime}$ be the list content of replica state, and we return $s^{\prime}$.

\section{C.5 Another Sequential Specification for list with addAt Interface}

Let us introduce another sequential specification Spec(addAt3) of list with addAt interface that also use tombstone. Each abstract state $\phi=(l, T)$ contains a sequence $l$ of elements of a given type and a set $T$ of elements appearing in the list. The element $l$ is the list of all input values, whether already removed or not; while $T$ stores the removed values and is used as tombstone. The sequential specification Spec(addAt3) of list with addAt interface is defined by:

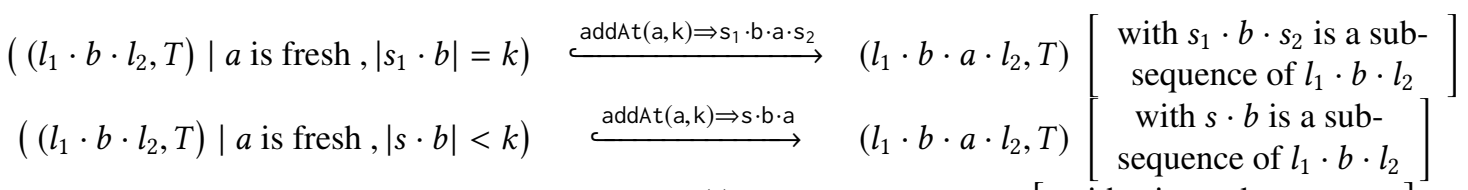

$$
\begin{aligned}
& ((l, T) \mid a \text { occurs in } l) \quad \stackrel{\text { remove }(\mathrm{a}) \Rightarrow \mathrm{s}}{\longrightarrow} \quad(l, T \cup\{a\})\left[\begin{array}{c}
\text { with } s \text { is a subsequence } \\
\text { of } l \text { and } a \notin s
\end{array}\right] \\
& (l, T) \quad \stackrel{\operatorname{read}() \Rightarrow(l / T)}{\longrightarrow} \quad(l, T)
\end{aligned}
$$

When we do addAt $(a, k)$ for an abstract state $(l, T)$, we do not try to check if there is a prefix of $l$ with length $k$ nor check if $|l / T|<k$. Instead, we check whether there exists a subsequence $l_{1}^{\prime} \cdot b \cdot l_{2}^{\prime}$ of $l$, such that $\left|l_{1}^{\prime} \cdot b\right|=k$, or check whether there exists a subsequence $l^{\prime} \cdot b$ of $l$, such that $\left|l^{\prime} \cdot b\right|<k$. Note that here we do not check the tombstone $T$. Intuitively, here $l_{1}^{\prime} \cdot b \cdot l_{2}^{\prime}$ or $l^{\prime} \cdot b$ is the list content of a replica, which is a local view. A intuitively explanation of RGA not being RA-linearizable w.r.t. 
Spec(addAt1) or Spec(addAt2) is that index is chosen locally to each replica and may be hard to have a global explanation. The fact that Spec(addAt3) also works in a "local" way may be a intuitive reason of RGA being RA-linearizable w.r.t. Spec(addAt3) (proved in the next subsection).

If $s_{1} \cdot b \cdot s_{2}$ is a subsequence of $l_{1} \cdot b \cdot l_{2}$ and $\left|s_{1} \cdot b\right|=k$, the method addAt $(a, k)$ puts $a$ immediately after $b$, and returns $s_{1} \cdot b \cdot a \cdot s_{2}$, assuming that each value is put into list at most once.

If $s \cdot b$ is a subsequence of $l_{1} \cdot b \cdot l_{2}$ and $|s \cdot b|<k$, the method addAt $(a, k)$ puts $a$ immediately after $b$, and returns $s \cdot b \cdot a$, assuming that each value is put into list at most once.

Method remove $(a)$ adds $a$ into $T$, hence removing $a$ from the list for subsequent calls to the read method. Finally, $\operatorname{read}() \Rightarrow s$ returns the list content excluding any element appearing in $T$.

\section{C.6 RGA is RA-linearizable w.r.t $\operatorname{Spec}(\operatorname{addAt3})$}

The following lemma states that RGA is RA-linearizable w.r.t. Spec(addAt3).

\section{LEMMA C.2. RGA is RA-linearizable w.r.t $\operatorname{Spec}($ addAt3).}

PROOF. Let us first prove Commutativity. By the causal delivery assumption and the preconditions of addAt, it cannot happen that an $\operatorname{addAt}\left(\mathrm{b},{ }_{-}\right) \Rightarrow_{-}$operation, which puts $\mathrm{b}$ after $\mathrm{a}$, is concurrent with an operation that adds a to the list, i.e., $\operatorname{addAt}\left(a, \_\right) \Rightarrow_{\ldots}$. Therefore, applying effectors of two concurrent addAt operations commute. By the causal delivery assumption and the preconditions of addAt and remove, it can not happen that an $\operatorname{addAt}\left(\mathrm{b}, \_\right) \Rightarrow{ }_{-}$operation adding $\mathrm{b}$ is concurrent with an operation remove $(b) \Rightarrow_{-}$that removes $b$. Threfore, applying effectors of a concurrent addAt operation and a concurrent remove operation commute. Since applying effectors of remove do set union to tombstone set $T$, applying effectors of concurrent remove operations commute.

Let us prove Refinement $t_{\mathrm{ts}}$. Let the refinement mapping abs be defined as: $\operatorname{abs}(N, T o m b)=(l, T)$, where $l=$ traverse $(\mathrm{N}, \emptyset)$, and $T=$ Tomb. Then,

- The fact that effectors of read queries are simulated by the corresponding operations of the specification is straightforward.

- Concerning effectors of $\ell=\operatorname{addAt}(\mathrm{a}, \mathrm{k}) \stackrel{\mathrm{ts} a}{\Rightarrow} \mathrm{s}$. we show that they are simulated by the corresponding specification operation $\operatorname{addAt}(\mathrm{a}, \mathrm{k}) \Rightarrow \mathrm{s}$ only when the timestamp $t s_{\mathrm{a}}$ is strictly greater than all the timestamps stored in the replica state where it applies. Thus, let (N, Tomb) be a replica state such that $t s<t s_{a}$ for every $t s$ with $\left({ }_{-}, t s,{ }_{-}\right) \in \mathrm{N}$.

Assume that when $\ell$ is generated from a replica state $\left(N_{\ell}, T o m b_{\ell}\right)$ and let $s_{\ell}=\operatorname{traverse}\left(N_{\ell}\right.$, Tomb $_{\ell}$ ),

- If $\left|s_{\ell}\right| \geq k$ : There exists $b \in s_{\ell}$ such that $\left|s_{1} \cdot b\right|=k$, where $s_{\ell}=s_{1} \cdot b \cdot s_{2}$. Then, $s=s_{1} \cdot b \cdot a \cdot s_{2}$. By the causal delivery assumption, all values of $s_{\ell}$ is in $N$ and is in $l$. Assume that $l=l_{1} \cdot b \cdot l_{2}$. Then, $s_{1} \cdot b \cdot s_{2}$ is a sub-sequence of $l_{1} \cdot b \cdot l_{2}$.

The result of applying the effector $\delta$ corresponding to $\operatorname{addAt}(\mathrm{a}, \mathrm{k}) \stackrel{\mathrm{ts}_{\mathrm{a}}}{\Rightarrow} \mathrm{s}$ is to add a as a child of $b$. Then, applying traverse on the new state will result in a sequence where a is placed just after $b$ because it has the biggest timestamp among the children of $b$ (and all the nodes in the tree $\mathrm{N}$ ). This corresponds exactly to the sequence obtained by applying the operation $\operatorname{add} A t(a, k) \Rightarrow s$ in the context of the specification.

- If $\left|s_{\ell}\right|<k$ : Let $b$ be the last value of $s_{\ell}$ and assume that $s_{\ell}=s_{1} \cdot b$. Then, $s=s_{1} \cdot b \cdot a$. By the causal delivery assumption, all values of $s_{\ell}$ is in $N$ and is in $l$. Assume that $l=l_{1} \cdot b \cdot l_{2}$. Then, $s_{1} \cdot b$ is a sub-sequence of $l_{1} \cdot b \cdot l_{2}$.

The result of applying the effector $\delta$ corresponding to $\operatorname{addAt}(\mathrm{a}, \mathrm{k}) \stackrel{\mathrm{ts} \mathrm{a}}{\Rightarrow} \mathrm{s}$ is to add a as a child of $b$. Then, applying traverse on the new state will result in a sequence where a is placed just after $b$ because it has the biggest timestamp among the children of $b$ (and all the nodes 
in the tree $\mathrm{N}$ ). This corresponds exactly to the sequence obtained by applying the operation $\operatorname{addAt}(\mathrm{a}, \mathrm{k}) \Rightarrow \mathrm{s}$ in the context of the specification.

- If $s_{\ell}=\epsilon$ : Let $b=\circ$. Then, $s=a$.

The result of applying the effector $\delta$ corresponding to $\operatorname{addAt}(\mathrm{a}, \mathrm{k}) \stackrel{\mathrm{ts} \mathrm{a}}{\Rightarrow} \mathrm{s}$ is to add a as a child of $b=\circ$. Then, applying traverse on the new state will result in a sequence where $a$ is the first value because it has the biggest timestamp among all the nodes in the tree N. This corresponds exactly to the sequence obtained by applying the operation $\operatorname{addAt}(a, k) \Rightarrow s$ in the context of the specification.

- Concerning effectors of $\ell=$ remove $(a) \Rightarrow \mathrm{s}$ : Assume that when $\ell$ is generated from a replica state $\left(N_{\ell}\right.$, Tomb $\left._{\ell}\right)$ and let $s_{\ell}=\operatorname{traverse}\left(N_{\ell}, \operatorname{Tomb}_{\ell}\right)$. Since $a \in s_{\ell}$, assume that $s_{\ell}=s_{\ell}^{1} \cdot a \cdot s_{\ell}^{2}$. We can see that $s=s_{\ell}^{1} \cdot s_{\ell}^{2}$.

By the causal delivery assumption we can see that all values of $\mathrm{N}_{\ell}$ are in $\mathrm{N}$. Therefore, $a \in l$ and $s_{\ell}^{1} \cdot s_{\ell}^{2}$ is a sub-sequence of $l$. The result of applying the effector $\delta$ corresponding to remove $(a) \Rightarrow s$ is to add a into Tomb. Then, applying traverse on the new state will result in a sequence without $a$. This corresponds exactly to the sequence obtained by applying the operation remove $(a) \Rightarrow s$ in the context of the specification.

This completes the proof of this lemma.

\section{RA-LINEARIZABILITY PROOF FOR STATE-BASED CRDT IMPLEMENTATIONS}

In this section, we briefly introduce the notion of state-based CRDT of [Shapiro et al. 2011], and propose its semantics. Then, we propose our prove methodology. Similarly as Sec. 4, our prove methodology intends to prove a similar lemma of Lemma 4.2 and Refinement. In state-based CRDT, a message contains a updated replica state instead of information of only one operation. Therefore, our proof is more complex.

We associate each operation with a "local" effector, which is only used for proof. Our prove methodology is used in three forms depending on whether the arguments of "local" effector of each update operation is unique.

\section{D.1 Brief Introduction of State-Based CRDT}

Let us first introduce the notion of join semilattice. Given a partial order $<$ and two values $x$ and $y$, we say that $z$ is a least upper bound of $x$ and $y$, if $x<z, y<z$, and there does not exists $z^{\prime}$, such that $\left(z^{\prime}<z\right) \wedge\left(x<z^{\prime}\right) \wedge\left(y<z^{\prime}\right)$. Let us use $x \sqcup y$ to denote the least upper bound of $x$ and $y$. A join semilattice is a partial order equipped with a least upper bound. According to the definition, it is obvious that the following properties hold:

$$
\begin{aligned}
& -x \sqcup y=y \sqcup x, \\
& \text { - } x \sqcup x=x, \\
& \text { - }(x \sqcup y) \sqcup z=x \sqcup(y \sqcup z) .
\end{aligned}
$$

Let us introduce the state-based crdt-implementation of [Shapiro et al. 2011], which is shown in Listing 6.

Each replica contains a copy of data and gives its initial value. Both query methods and update methods can have arguments and return values, and can check pre-condition before a method is executed. Note that both query methods and update methods executes locally without synchronization with other replica. Instead, nondeterministically, state-based CRDT transmit modified payload as message between replicas. When a replica receives a message of modified payload, it calls method merge, which takes the current payload and the payload in the message, and returns a new payload. The intuition of state-based CRDT is that, the domain of replica state is a join semilattice; the order 
of the join semilattice is given in compare method; $\operatorname{merge}(x, y)$ returns the least upper bound of the join semilattice.

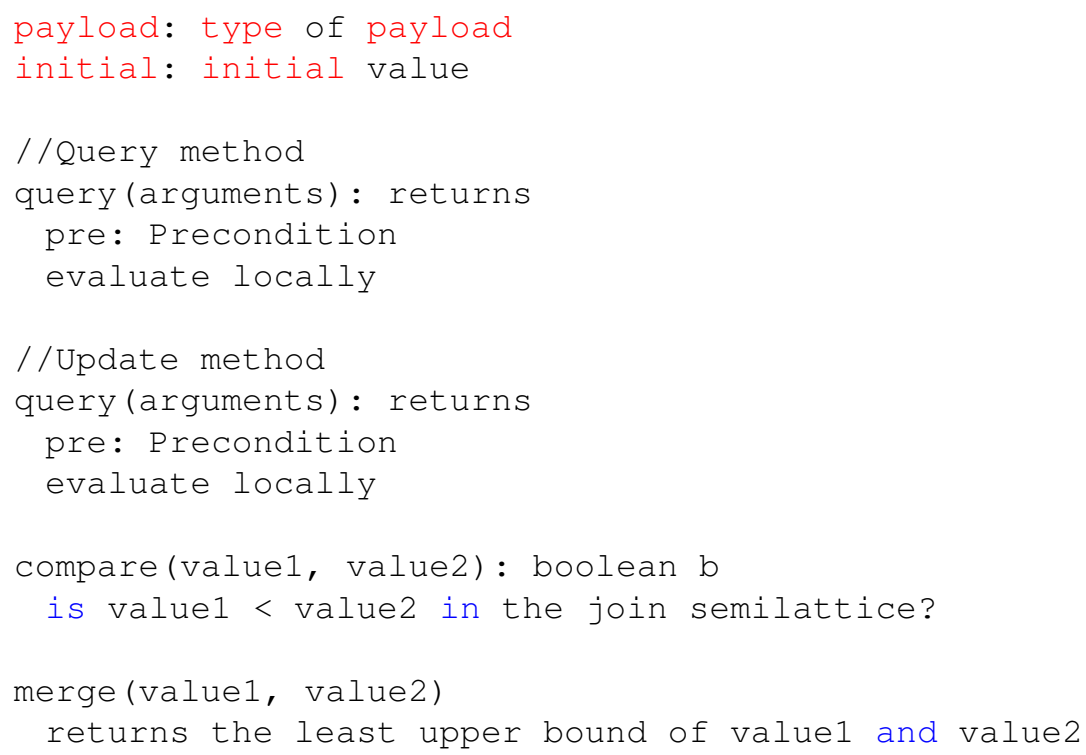

Listing 6. Outline of state-based CRDT

\section{D.2 Semantics of State-Based CRDT Implementations}

Given a state-based CRDT object o, its semantics is defined as a labeled transition system (LTS) $\llbracket \mathrm{o} \rrbracket_{s}=\left(\mathrm{GC}, \mathbb{A}, \mathrm{gc}_{0}, \rightarrow\right)$, where $\mathrm{GC}$ is a set of global configurations, $\mathbb{A}$ is the set of transition labels, $\mathrm{gc}_{0}$ is the initial configuration, and $\rightarrow \subseteq \mathrm{GC} \times \mathbb{A} \times \mathrm{GC}$ is the transition relation.

A global configuration (G, vis, Ms) is a "snapshot" of the system that records all the operations that have been executed. $G \in[\mathbb{R} \rightarrow \mathrm{LC}]$ stores the local configuration of each replica, and vis $\subseteq \mathcal{P}(\mathbb{L} \times \mathbb{L})$ is the visibility relation. A local configuration $(\mathrm{L}, \sigma)$ contains the state $\sigma$ of a replica and the set $\mathrm{L}$ of labels of operations that are "visible" to current replica. $\ell \in \mathrm{L}$, if either $\ell$ originates in this replica, or there exists a message $\left(L s^{\prime}, \sigma^{\prime}\right)$ that is "aware of $\ell$ ", or we can say, $\ell \in L s^{\prime}$, and such message has been applied in current replica state. Ms stores the messages in current distributed system. Each message of $M s$ is a local configuration $(L s, \sigma)$. The reason for storing $L s$ in a message is to construct the visibility relation.

The transition labels are operations. For some fixed initial replica state $\sigma_{0}$, the initial global configuration is defined by $\mathrm{gc}_{0}=\left(\mathrm{G}_{0}, \emptyset, \emptyset\right) \in \mathrm{GC}$, where $\mathrm{G}_{0}$ maps each replica $r$ into $\left(\emptyset, \sigma_{0}\right)$.

The transition relation between global configurations is defined as follows:

The first transition rule OPERATION describes a replica $r$ in state $\sigma$ executing an invocation of method $\mathrm{m}$ with argument $a$. We use a function $\theta$ to represent the behavior of the method. $\theta(\sigma, \mathrm{m}, a)$ stands for calling method $\mathrm{m}$ with argument $a$ on the replica state $\sigma$, and then results in a return value $b$, a new replica state $\sigma^{\prime}$, and possibly, a timestamp $t s$. We assume that timestamps are consistent with the visibility relation vis. After this transition, the local configuration $(\mathrm{L}, \sigma)$ of $r$ is changed by modifying replica state into $\sigma^{\prime}$ and adding $\ell$ into the set $L$ of labels; the visibility relation vis is changed to record the fact that all operations in $L$ is now visible to $\ell$. 


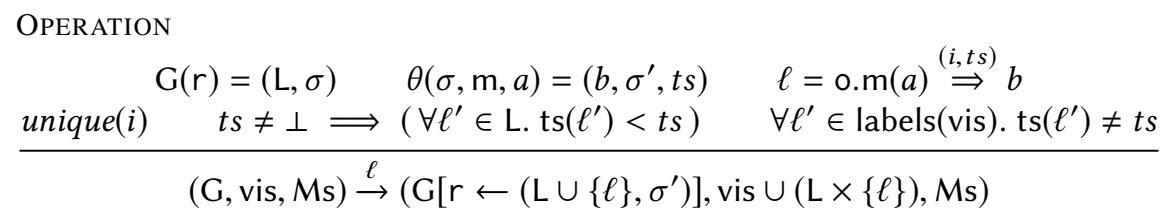

The second rule GENERATE describes a replica $r$ sending a message. The content of this message is the local configuration $(\mathrm{L}, \sigma)$ of current replica. After this transition, only the set Ms of messages is changed by adding this message.

$$
\frac{\text { Generate }}{\mathrm{G}(\mathrm{r})=(\mathrm{L}, \sigma)}
$$

The third rule APPLY describes a replica $r$ with local configuration $\left(\mathrm{L}, \sigma_{1}\right)$ applying a message $\left(L s, \sigma_{2}\right)$. This transition results in modifying the state of $r$ to merge $\left(\sigma_{1}, \sigma_{2}\right)$ and adding $L s$ to the set of operations of local configuration of replica $r$.

$$
\frac{\mathrm{G}(\mathrm{r})=\left(\mathrm{L}, \sigma_{1}\right) \quad\left(L s, \sigma_{2}\right) \in \mathrm{Ms}}{(\mathrm{G}, \mathrm{vis}, \mathrm{Ms}) \rightarrow\left(\mathrm{G}\left[\mathrm{r} \leftarrow\left(\mathrm{L} \cup L s, \operatorname{merge}\left(\sigma_{1}, \sigma_{2}\right)\right)\right], \text { vis, Ms }\right)}
$$

It is clear from the definition of the transition relation that, vis is a strict partial order.

A message can be applied multiple times on a same replica, or can be lost, or can be applied in any order.

\section{D.3 Proof Methodology for the Uniquely-Identified-Effectors}

"Local" Effectors and apply method: In state-based CRDT, messages sent between replicas contain replica states instead of operations. To be able to prove a similar result as in Lemma 4.2, we extend each method as follows: For each method, the reading part is called a generator, and the updating part is called a "local" effector. Here we use "local" to emphasize that such effectors are only applied at the original replica, they are a proof artifact with no implications on the state-based CRDT semantics.

As in the operation based CRDTs, the "local" effector uses as arguments values produced by the generator. We give examples in Section E. Let $\arg (\ell)$ be the arguments that are generated by the generator of $\ell$. We define a "universal local effector function" apply $(\sigma, \arg (\ell))$, which is a replica state transformer. $\operatorname{apply}(\sigma, \arg (\ell))$ works as applying the "local" effector of $\ell$ on $\sigma$. The arguments of "local" effectors are important in our proof, and this is the reason why we write them explicitly in defining apply.

Since the "local" effector contains information of only one operation, applying the "local" effector is normally "simpler" than merge.

Three Cases: We consider three different cases of state-based CRDT as follows:

- Uniquely-Identified-Effectors: The argument of "local" effector of each update operation is unique. Moreover, there is a partial order among arguments of "local" effectors, and such partial order is consistent with the visibility relation.

- Cumulative Effectors: For each update operations $\ell, \ell^{\prime}, \arg (\ell)=\arg \left(\ell^{\prime}\right)$, if and only if $\ell$ and $\ell^{\prime}$ use a same method, same input values, same return values, and originate in a same replica.

- Idempotent Effectors: For each update operations $\ell, \ell^{\prime}, \arg (\ell)=\arg \left(\ell^{\prime}\right)$, if and only if $\ell$ and $\ell^{\prime}$ use a same method, same input values and same return values. 
Uniquely-identified-effectors contains state-based multi-value register and state-based LWWelement-set. Cumulative effectors contains state-based PN-counter. Idempotent effectors contains state-based 2P-set.

In this subsection, we propose our proof methodology of RA-linearizability for the uniquelyidentified-effectors.

A Lemma Similar to Lemma 4.2: We need to prove the following lemma, which states that $\sigma$ can be obtained from the initial replica state by applying "local" effectors of visible operations in any order consistent with the visibility relation. Especially, this holds when this order is the projection of linearization into visible operations, since the linearization is consistent with the visibility relation. Here Prop 1, Prop $_{2}$, Prop $_{3}$, Prop $_{4}$ and Prop $_{5}$ are properties that are introduced in the latter part of this subsection. We will prove that they make Lemma D.1 holds.

LEMMA D.1. Let o be a state-based CRDT that is uniquely-identified-effectors, and satisfies

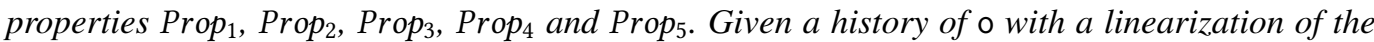
operations in history (possibly, rewritten using a query-update rewriting $\gamma$ ), consistent with the visibility relation. Then, for each local configuration $(\mathrm{L}, \sigma)$ or message with content $(\mathrm{L}, \sigma)$,

$$
\sigma=\operatorname{apply}\left(\operatorname{apply}\left(\ldots \operatorname{apply}\left(\sigma_{0}, \arg \left(\ell_{1}\right)\right), \ldots, \arg \left(\ell_{k}\right)\right)\right.
$$

where $\sigma_{0}$ is the initial state, $\mathrm{L}=\left\{\ell_{1}, \ldots, \ell_{k}\right\}$, and $\ell_{1} \cdot \ldots \ell_{k}$ is consistent with the visibility relation. Especially, this holds when $\ell_{1} \cdot \ldots \cdot \ell_{k}=$ lin $\downarrow_{L}$.

Definition of Predicate $P 1$ and Properties Prop $_{1}, \ldots$, Prop $p_{5}$ : Let us formally introduce the necessary predicate and properties for proving Lemma D.1.

We define a predicate $P 1$ that has the following property: $P 1(\sigma, \arg (\ell))$ holds, if and only if, if $\sigma=\operatorname{apply}\left(\operatorname{apply}\left(\ldots \operatorname{apply}\left(\sigma_{0}, \arg \left(\ell_{1}\right)\right), \ldots, \arg \left(\ell_{n}\right)\right)\right.$ for some operations $\ell_{1}, \ldots, \ell_{n}$, then for each $1 \leq i \leq n, \arg (\ell)$ is not less than $\arg \left(\ell_{i}\right)$.

Let us propose the following properties $\operatorname{Prop}_{1}, \ldots$, Prop $_{5}$ :

- $\operatorname{Prop}_{1}$ : If operations $\ell$ and $\ell^{\prime}$ are concurrent, then for each replica state $\sigma, \operatorname{apply}(\operatorname{apply}(\sigma, \arg (\ell))$, $\left.\arg \left(\ell^{\prime}\right)\right)=\operatorname{apply}\left(\operatorname{apply}\left(\sigma, \arg \left(\ell^{\prime}\right)\right), \arg (\ell)\right)$

- Prop 2 : If $P 1(\sigma, \arg (\ell))$ and $P 1\left(\sigma^{\prime}, \arg (\ell)\right)$ holds, then merge $\left(\sigma, \operatorname{apply}\left(\sigma^{\prime}, \arg (\ell)\right)\right)=\operatorname{apply}($ merge $\left.\left(\sigma, \sigma^{\prime}\right), \arg (\ell)\right)$.

- Prop $:$ If $P 1(\sigma, \arg (\ell))$ and $P 1\left(\sigma^{\prime}, \arg (\ell)\right)$ holds, then merge $\left(\operatorname{apply}(\sigma, \arg (\ell)), \operatorname{apply}\left(\sigma^{\prime}, \arg (\ell\right.\right.$ )$)=\operatorname{apply}\left(\operatorname{merge}\left(\sigma, \sigma^{\prime}\right), \arg (\ell)\right)$.

- Prop 4 : merge $\left(\sigma_{0}, \sigma_{0}\right)=\sigma_{0}$. For each replica state $\sigma, \sigma^{\prime}$, merge $\left(\sigma, \sigma^{\prime}\right)=\operatorname{merge}\left(\sigma^{\prime}, \sigma\right)$.

- Prop 5 : During the execution, if the current replica state of a replica is $\sigma$, this replica does operation $\ell$ and changes the replica state into $\sigma^{\prime}$. Then, $\operatorname{apply}(\sigma, \arg (\ell))=\sigma^{\prime}$.

\section{Proof of Lemma D.1:}

ProOF. We prove by induction on executions. Obvious they hold in $\mathrm{gc}_{0}$. Assume they hold along the execution $\mathrm{gc}_{0} \rightarrow^{*} \mathrm{gc}$ and there is a new transition $\mathrm{gc} \rightarrow \mathrm{gc}^{\prime}$. We need to prove that they still hold in $g c^{\prime}$.

- For case when replica $r$ do operation $\ell$ : Let $(L, \sigma)$ and $\left(\mathrm{L}^{\prime}, \sigma^{\prime}\right)$ be the local configuration of replica $r$ of gc and $g c^{\prime}$, respectively. By the semantics we have $L^{\prime}=L \cup\{\ell\}$. We need to prove that this lemma holds for local configuration $\left(\mathrm{L}^{\prime}, \sigma^{\prime}\right)$.

By the induction assumption, we know that $\sigma=\operatorname{apply}\left(\operatorname{apply}\left(\ldots \operatorname{apply}\left(\sigma_{0}, \arg \left(\ell_{1}\right)\right), \ldots, \arg (\right.\right.$ $\left.\ell_{n}\right)$ ), where $\mathrm{L}=\left\{\ell_{1}, \ldots, \ell_{n}\right\}$ and $\ell_{1} \cdot \ldots \cdot \ell_{n}$ is consistent with the visibility relation. By $\operatorname{Prop}_{5}$ we can see that $\sigma^{\prime}=\operatorname{apply}(\sigma, \arg (\ell))$. By $\operatorname{Prop}_{1}$, we can see that for each permutation $\ell_{1}^{\prime} \cdot \ldots \cdot \ell_{n+1}^{\prime}$ of $\ell_{1} \cdot \ldots \cdot \ell_{n} \cdot \ell$ that is consistent with visibility relation, we have that 
$\sigma=\operatorname{apply}\left(\operatorname{apply}\left(\ldots \operatorname{apply}\left(\sigma_{0}, \arg \left(\ell_{1}^{\prime}\right)\right), \ldots, \arg \left(\ell_{n+1}^{\prime}\right)\right)\right.$. Therefore, this lemma still holds for local configuration $\left(\mathrm{L}^{\prime}, \sigma^{\prime}\right)$.

- For case when replica $r$ send a message: Let $(L, \sigma)$ and $\left(\mathrm{L}^{\prime}, \sigma^{\prime}\right)$ be the local configuration of replica $r$ of gc and gc', respectively. By the semantics we have $\left(\mathrm{L}^{\prime}, \sigma^{\prime}\right)=(\mathrm{L}, \sigma)$, and the content of the message is $(\mathrm{L}, \sigma)$. We need to prove that this lemma holds for the message with content $(\mathrm{L}, \sigma)$. This is obvious since this lemma holds for the local configuration $(\mathrm{L}, \sigma)$ by the induction assumption.

- For case when replica $r$ apply a message: Let $(L, \sigma)$ and $\left(\mathrm{L}^{\prime}, \sigma^{\prime}\right)$ be the local configuration of replica $r$ of gc and $g c^{\prime}$, respectively. Let $\left(\mathrm{L}^{\prime \prime}, \sigma^{\prime \prime}\right)$ be the content of the message. We need to prove that this lemma still holds for local configuration $\left(\mathrm{L}^{\prime}, \sigma^{\prime}\right)$. By the semantics we have that $\mathrm{L}^{\prime}=\mathrm{L} \cup \mathrm{L}^{\prime \prime}$ and $\sigma^{\prime}=\operatorname{merge}\left(\sigma, \sigma^{\prime \prime}\right)$.

By the induction assumption, we know that $\sigma=\operatorname{apply}\left(\operatorname{apply}\left(\ldots \operatorname{apply}\left(\sigma_{0}, \arg \left(\ell_{1}\right)\right), \ldots, \arg (\right.\right.$ $\left.\left.\ell_{u}\right)\right), \sigma^{\prime \prime}=\operatorname{apply}\left(\operatorname{apply}\left(\ldots \operatorname{apply}\left(\sigma_{0}, \arg \left(\ell_{1}^{\prime \prime}\right)\right), \ldots, \arg \left(\ell_{v}^{\prime \prime}\right)\right)\right.$, where $\mathrm{L}=\left\{\ell_{1}, \ldots, \ell_{u}\right\}, \mathrm{L}^{\prime \prime}=$ $\left\{\ell_{1}^{\prime \prime}, \ldots, \ell_{v}^{\prime \prime}\right\}$, and $\ell_{1} \cdot \ldots \cdot \ell_{u}$ and $\ell_{1}^{\prime \prime} \cdot \ldots \cdot \ell_{v}^{\prime \prime}$ are consistent with the visibility relation.

Then, our proof proceed as follows:

- If $\mathrm{L} \neq \mathrm{L}^{\prime \prime}$ : There exists an operation $\ell_{a}$ that is a maximal operation of $\mathrm{L} \cup \mathrm{L}^{\prime \prime}$ w.r.t the partial order of arguments of "local" effectors, and $\left(\ell_{a} \in \mathrm{L}\right.$ " $\left.\backslash \mathrm{L}\right) \vee\left(\ell_{a} \in \mathrm{L} \backslash \mathrm{L}^{\prime \prime}\right)$.

- If $\ell_{a} \in \mathrm{L}^{\prime \prime} \backslash \mathrm{L}$ : Assume that $\ell_{a}=\ell_{i d x}^{\prime \prime}$. Since $\ell_{1}^{\prime \prime} \cdot \ldots \cdot \ell_{v}^{\prime \prime}$ is consistent with the visibility relation, we can see that $\forall j>i d x, \ell_{i d x}^{\prime \prime}$ and $\ell_{j}^{\prime \prime}$ are concurrent. We can obtain $\ell_{1}^{\prime \prime} \cdot \ldots \cdot \ell_{i d x-1}^{\prime \prime}$. $\ell_{i d x+1}^{\prime \prime} \cdot \ldots \cdot \ell_{v}^{\prime \prime} \cdot \ell_{i d x}^{\prime \prime}$ from $\ell_{1}^{\prime \prime} \cdot \ldots \cdot \ell_{v}^{\prime \prime}$ by several times of swapping pairs of operations that are concurrent and adjacent. Let $\sigma_{1}^{\prime \prime}=\operatorname{apply}\left(\operatorname{apply}\left(\ldots \operatorname{apply}\left(\sigma_{0}, \arg \left(\ell_{1}^{\prime \prime}\right)\right), \ldots, \arg \left(\ell_{i d x-1}^{\prime \prime}\right)\right)\right.$. $\left.\left.\arg \left(\ell_{i d x+1}^{\prime \prime}\right)\right) \cdot \ldots \cdot \arg \left(\ell_{v}^{\prime \prime}\right)\right)$. By $\operatorname{Prop}_{1}$, we can see that $\sigma^{\prime \prime}=\operatorname{apply}\left(\sigma_{1}^{\prime \prime}, \arg \left(\ell_{i d x}^{\prime \prime}\right)\right)$.

Since $\ell_{a}=\ell_{i d x}^{\prime \prime}$ is a maximal operation of $\mathrm{L} \cup \mathrm{L}^{\prime \prime}$ w.r.t the partial order of arguments of "local" effectors, we can see that $P 1\left(\sigma, \arg \left(\ell_{i d x}^{\prime \prime}\right)\right)$ and $P 1\left(\sigma_{1}^{\prime \prime}, \arg \left(\ell_{i d x}^{\prime \prime}\right)\right)$ hold. By $\operatorname{Prop}_{2}$, we can see that $\sigma^{\prime}=\operatorname{merge}\left(\sigma, \sigma^{\prime \prime}\right)=\operatorname{merge}\left(\sigma, \operatorname{apply}\left(\sigma_{1}^{\prime \prime}, \arg \left(\ell_{i d x}^{\prime \prime}\right)\right)\right)=\operatorname{apply}($ $\left.\operatorname{merge}\left(\sigma, \sigma_{1}^{\prime \prime}\right), \arg \left(\ell_{i d x}^{\prime \prime}\right)\right)$.

- If $\ell_{a} \in \mathrm{L} \backslash \mathrm{L}^{\prime \prime}:$ By Prop4, we can see that $\sigma^{\prime}=\operatorname{merge}\left(\sigma, \sigma^{\prime \prime}\right)=\operatorname{merge}\left(\sigma^{\prime \prime}, \sigma\right)$. Then, we can similarly work as above case.

- If $\mathrm{L}=\mathrm{L}^{\prime \prime}$ : There exists an operation $\ell_{a}$ that is a maximal operation of $\mathrm{L} \cup \mathrm{L}^{\prime \prime}$ w.r.t the partial order of "local" effectors.

Assume that $\ell_{a}=\ell_{i d x 1}$ and $\ell_{a}=\ell_{i d x 2}^{\prime \prime}$. Similarly, we can prove that $\sigma=\operatorname{apply}\left(\sigma_{1}, \arg \left(\ell_{a}\right)\right)$ and $\sigma^{\prime \prime}=\operatorname{apply}\left(\sigma_{1}^{\prime \prime}, \arg \left(\ell_{a}\right)\right)$, where $\sigma_{1}=\operatorname{apply}\left(\operatorname{apply}\left(\ldots \operatorname{apply}\left(\sigma_{0}, \arg \left(\ell_{1}\right)\right), \ldots, \arg (\right.\right.$ $\left.\left.\left.\left.\ell_{i d x 1-1}\right)\right) \cdot \arg \left(\ell_{i d x 1+1}\right)\right) \cdot \ldots \cdot \arg \left(\ell_{u}\right)\right)$ and $\sigma_{1}^{\prime \prime}=\operatorname{apply}\left(\operatorname{apply}\left(\ldots \operatorname{apply}\left(\sigma_{0}, \arg \left(\ell_{1}^{\prime \prime}\right)\right), \ldots, \arg (\right.\right.$ $\left.\left.\left.\left.\ell_{i d x 2-1}^{\prime \prime}\right)\right) \cdot \arg \left(\ell_{i d x 2+1}^{\prime \prime}\right)\right) \cdot \ldots \cdot \arg \left(\ell_{v}^{\prime \prime}\right)\right)$.

Since $\ell_{a}$ is a maximal operation of $L \cup L^{\prime \prime}$ w.r.t the partial order of arguments of "local" effectors, we can see that $P 1\left(\sigma_{1}, \arg \left(\ell_{a}\right)\right)$ and $P 1\left(\sigma_{1}^{\prime \prime}, \arg \left(\ell_{a}\right)\right)$ hold. By $P r o p_{3}$, we can see that $\sigma^{\prime}=\operatorname{merge}\left(\sigma, \sigma^{\prime \prime}\right)=\operatorname{merge}\left(\operatorname{apply}\left(\sigma_{1}, \arg \left(\ell_{a}\right)\right), \operatorname{apply}\left(\sigma_{1}^{\prime \prime}, \arg \left(\ell_{a}\right)\right)\right)=\operatorname{apply}\left(\operatorname{merge}\left(\sigma_{1}, \sigma_{1}^{\prime \prime}\right)\right.$, $\left.\arg \left(\ell_{a}\right)\right)$.

By doing above process for several times, we finally can prove that, $\sigma^{\prime}=\operatorname{apply}(\operatorname{apply}(\ldots \operatorname{apply}($ merge $\left.\left.\left(\sigma_{0}, \sigma_{0}\right), \arg \left(\ell_{1}^{\prime}\right)\right), \ldots, \arg \left(\ell_{n}^{\prime}\right)\right)$, where $\mathrm{L}^{\prime}=\left\{\ell_{1}^{\prime}, \ldots, \ell_{n}^{\prime}\right\}$, and for each $i, \arg \left(\ell_{i}^{\prime}\right)$ is maximal among $\left\{\arg \left(\ell_{1}^{\prime}\right), \ldots, \arg \left(\ell_{i}^{\prime}\right)\right\}$ w.r.t the partial order of arguments of "local" effectors. Let us prove that $\ell_{1}^{\prime} \cdot \ldots \cdot \ell_{n}^{\prime}$ is consistent with the visibility relation by contradiction. Assume that there exists indexes $i 1<i 2$, such that $\left(\ell_{i 2}^{\prime}, \ell_{i 1}^{\prime}\right) \in$ vis. Since the partial order of arguments of "local" effectors is consistent with the visibility relation, we can see that $\arg \left(\ell_{i 2}^{\prime}\right)<\arg \left(\ell_{i 1}^{\prime}\right)$. However, we already knows that $\arg \left(\ell_{i 2}^{\prime}\right)$ is maximal among $\left\{\arg \left(\ell_{1}^{\prime}\right), \ldots, \arg \left(\ell_{i 1}^{\prime}\right), \ldots, \arg \left(\ell_{i 2}^{\prime}\right)\right\}$ w.r.t the partial order of arguments of "local" effectors, which is a contradiction. Therefore, $\ell_{1}^{\prime} \cdot \ldots \cdot \ell_{n}^{\prime}$ is consistent with the visibility relation. 
By $\operatorname{Prop}_{4}$, we can see that $\sigma^{\prime}=\operatorname{apply}\left(\operatorname{apply}\left(\ldots \operatorname{apply}\left(\sigma_{0}, \arg \left(\ell_{1}^{\prime}\right)\right), \ldots, \arg \left(\ell_{n}^{\prime}\right)\right)\right.$. By $\operatorname{Prop}_{1}$, we can see that, for each permutation $\ell_{1}^{1} \cdot \ldots \cdot \ell_{n}^{1}$ of $\ell_{1}^{\prime} \cdot \ldots \cdot \ell_{n}^{\prime}$ that is consistent with visibility relation, we have that $\sigma=\operatorname{apply}\left(\operatorname{apply}\left(\ldots \operatorname{apply}\left(\sigma_{0}, \arg \left(\ell_{1}^{1}\right)\right), \ldots, \arg \left(\ell_{n}^{1}\right)\right)\right.$. Therefore, this lemma still holds for local configuration $\left(\mathrm{L}^{\prime}, \sigma^{\prime}\right)$.

This completes the proof of this lemma.

Proving Refinement: Since our method has generator and "local" effector, similarly as Sec. 3, when the state-based CRDT object admits timestamp-order linearizations, we prove the existence of a refinement mapping by proving Refinement $t_{\mathrm{ts}}$. Then, together with Lemma D.1, we can prove RA-linearizability.

For the state-based multi-value register (introduced in Sec. E.1), each write method generates an unique version vector. Such version vector is contained in the arguments of "local" effectors of write, and the partial order of version vectors are consistent with the visibility relation. The execution-order linearization is consistent with the partial order of version vectors. The state-based multi-value register admits execution-order linearizations. Then, we define Refinement $_{v}$ as the existence of a mapping abs such that:

Simulating effectors: For every effector $\delta$ corresponding to an operation $\ell$,

$$
\forall \sigma \in \Sigma . P 1(\sigma, \ell) \wedge \sigma^{\prime}=\delta(\sigma) \Longrightarrow \operatorname{abs}(\sigma) \stackrel{\operatorname{upd}(\gamma(\ell))}{\longrightarrow} \operatorname{abs}\left(\sigma^{\prime}\right)
$$

Simulating generators: For every query $\mathrm{m}$, and every $\sigma \in \Sigma$,

$$
\theta(\sigma, \mathrm{m}, a)=\left(b,,_{-}\right) \Longrightarrow \operatorname{abs}(\sigma) \stackrel{\ell}{\hookrightarrow} \operatorname{abs}(\sigma)
$$

where $\ell=\mathrm{m}(a) \Rightarrow b$. Also, for every query-update $\mathrm{m}$, and $\sigma \in \Sigma$,

$$
\theta(\sigma, \mathrm{m}, a)=\left(b,,_{-}\right) \Longrightarrow \operatorname{abs}(\sigma) \stackrel{\operatorname{qry}(\gamma(\ell))}{\longrightarrow} \operatorname{abs}(\sigma) .
$$

We need to prove Refinement ${ }_{v}$. Then, together with Lemma D.1, we can prove RA-linearizability.

\section{D.4 Proof Methodology for the Cumulative Effectors}

In this subsection, we propose our prove methodology of RA-linearizability for the cumulative effectors.

A Lemma Similar to Lemma 4.2: We need to prove the following lemma, which states that $\sigma$ can be obtained from the initial replica state by applying "local" effectors of visible operations in any order. Especially, this holds when this order is the projection of linearization into visible operations. Here Prop ${ }_{1}^{\prime}$, Prop ${ }_{2}^{\prime}$ and Prop $_{3}^{\prime}$ are properties that are introduced in the latter part of this subsection. They make Lemma D. 2 holds.

LEMMA D.2. Let o be a state-based CRDT that is cumulative effectors, and satisfies properties Prop $_{1}^{\prime}$, Prop ${ }_{2}^{\prime}$, Prop $_{3}^{\prime}$, Prop ${ }_{4}$ and Prop ${ }_{5}$. Given a history of o with a linearization of the operations in history (possibly, rewritten using a query-update rewriting $\gamma$ ), consistent with the visibility relation. Then, for each local configuration $(\mathrm{L}, \sigma)$ or message with content $(\mathrm{L}, \sigma)$,

$$
\sigma=\operatorname{apply}\left(\operatorname{apply}\left(\ldots \operatorname{apply}\left(\sigma_{0}, \arg \left(\ell_{1}\right)\right), \ldots, \arg \left(\ell_{k}\right)\right)\right.
$$

where $\sigma_{0}$ is the initial state and $\mathrm{L}=\left\{\ell_{1}, \ldots, \ell_{k}\right\}$. Especially, this holds when $\ell_{1} \cdot \ldots \cdot \ell_{k}=\operatorname{lin} \downarrow_{L}$.

Definition of Predicate $P 2$ and Properties Prop $_{1}^{\prime}$, Prop $p_{2}^{\prime}$, Prop $p_{3}^{\prime}$ : Let us formally introduce the necessary predicate and properties for proving Lemma D.2. 
We define a predicate $P_{2}$ that that has the following property: $P 2(\sigma, \arg (\ell))$ holds, if and only if, if $\sigma=\operatorname{apply}\left(\operatorname{apply}\left(\ldots \operatorname{apply}\left(\sigma_{0}, \arg \left(\ell_{1}\right)\right), \ldots, \arg \left(\ell_{n}\right)\right)\right.$ for some operations $\ell_{1}, \ldots, \ell_{n}$, then for each $1 \leq i \leq n, \arg (\ell) \neq \arg \left(\ell_{i}\right)$.

Let us propose the following properties $\operatorname{Prop}_{1}^{\prime}, \operatorname{Prop}_{2}^{\prime}$ and $\operatorname{Prop}_{3}^{\prime}$ :

- Prop ${ }_{1}^{\prime}$ : For each replica state $\sigma, \operatorname{apply}\left(\operatorname{apply}(\sigma, \arg (\ell)), \arg \left(\ell^{\prime}\right)\right)=\operatorname{apply}\left(\operatorname{apply}\left(\sigma, \arg \left(\ell^{\prime}\right)\right)\right.$, $\arg (\ell))$.

- Prop $p_{2}^{\prime}$ : If $P 2(\sigma, \arg (\ell))$ and $P 2\left(\sigma^{\prime}, \arg (\ell)\right)$ hold, then merge $\left(\sigma, \operatorname{apply}\left(\sigma^{\prime}, \arg (\ell)\right)\right)=\operatorname{apply}($ merge $\left.\left(\sigma, \sigma^{\prime}\right), \arg (\ell)\right)$.

- Prop $p_{3}^{\prime}$ : For each replica state $\sigma, \sigma^{\prime}$ and argument $x$ of a "local" effector, merge $(\operatorname{apply}(\sigma, x)$, $\operatorname{apply}\left(\sigma^{\prime}, x\right)=\operatorname{apply}\left(\operatorname{merge}\left(\sigma, \sigma^{\prime}\right), x\right)$.

\section{Proof of Lemma D.2:}

Proof. We prove by induction on executions. Obvious they hold in $\mathrm{gc}_{0}$. Assume they hold along the execution $\mathrm{gc}_{0} \rightarrow^{*} \mathrm{gc}$ and there is a new transition $\mathrm{gc} \rightarrow \mathrm{gc}^{\prime}$. We need to prove that they still hold in $\mathrm{gc}^{\prime}$.

- For case when replica $r$ do operation $\ell$ : Let $(\mathrm{L}, \sigma)$ and $\left(\mathrm{L}^{\prime}, \sigma^{\prime}\right)$ be the local configuration of replica $r$ of gc and $g c^{\prime}$, respectively. By the semantics we have $L^{\prime}=L \cup\{\ell\}$. We need to prove that this lemma holds for local configuration $\left(\mathrm{L}^{\prime}, \sigma^{\prime}\right)$.

By the induction assumption, we know that $\sigma=\operatorname{apply}\left(\operatorname{apply}\left(\ldots \operatorname{apply}\left(\sigma_{0}, \arg \left(\ell_{1}\right)\right), \ldots, \arg (\right.\right.$ $\left.\ell_{n}\right)$ ), where $\mathrm{L}=\left\{\ell_{1}, \ldots, \ell_{n}\right\}$. By $\operatorname{Prop}_{5}$ we can see that $\sigma^{\prime}=\operatorname{apply}(\sigma, \arg (\ell))$. By $\operatorname{Prop}_{1}^{\prime}$, we can see that for each permutation $\ell_{1}^{\prime} \cdot \ldots \cdot \ell_{n+1}^{\prime}$ of $\ell_{1} \cdot \ldots \cdot \ell_{n} \cdot \ell$, we have that $\sigma=$ $\operatorname{apply}\left(\operatorname{apply}\left(\ldots \operatorname{apply}\left(\sigma_{0}, \arg \left(\ell_{1}^{\prime}\right)\right), \ldots, \arg \left(\ell_{n+1}^{\prime}\right)\right)\right.$. Therefore, this lemma still holds for local configuration $\left(\mathrm{L}^{\prime}, \sigma^{\prime}\right)$.

- For case when replica $r$ send a message: Let $(L, \sigma)$ and $\left(\mathrm{L}^{\prime}, \sigma^{\prime}\right)$ be the local configuration of replica $r$ of gc and $g c^{\prime}$, respectively. By the semantics we have $\left(\mathrm{L}^{\prime}, \sigma^{\prime}\right)=(\mathrm{L}, \sigma)$, and the content of the message is $(\mathrm{L}, \sigma)$. We need to prove that this lemma holds for the message with content $(\mathrm{L}, \sigma)$. This is obvious since this lemma holds for the local configuration $(\mathrm{L}, \sigma)$ by the induction assumption.

- For case when replica $r$ apply a message: Let $(L, \sigma)$ and $\left(L^{\prime}, \sigma^{\prime}\right)$ be the local configuration of replica $r$ of gc and $g c^{\prime}$, respectively. Let $\left(\mathrm{L}^{\prime \prime}, \sigma^{\prime \prime}\right)$ be the content of the message. We need to prove that this lemma still holds for local configuration $\left(\mathrm{L}^{\prime}, \sigma^{\prime}\right)$. By the semantics we have $\mathrm{L}^{\prime}=\mathrm{L} \cup \mathrm{L}^{\prime \prime}$ and $\sigma^{\prime}=\operatorname{merge}\left(\sigma, \sigma^{\prime \prime}\right)$.

By the induction assumption, we know that $\sigma=\operatorname{apply}\left(\operatorname{apply}\left(\ldots \operatorname{apply}\left(\sigma_{0}, \arg \left(\ell_{1}\right)\right), \ldots, \arg (\right.\right.$ $\left.\left.\ell_{u}\right)\right), \sigma^{\prime \prime}=\operatorname{apply}\left(\operatorname{apply}\left(\ldots \operatorname{apply}\left(\sigma_{0}, \arg \left(\ell_{1}^{\prime \prime}\right)\right), \ldots, \arg \left(\ell_{v}^{\prime \prime}\right)\right)\right.$, where $\mathrm{L}=\left\{\ell_{1}, \ldots, \ell_{u}\right\}$ and $\mathrm{L}^{\prime \prime}=\left\{\ell_{1}^{\prime \prime}, \ldots, \ell_{v}^{\prime \prime}\right\}$.

Let us "move the local effectors of duplicate operations out". If $\mathrm{L} \cap \mathrm{L}$ " $\neq \emptyset$ : Let $\ell_{a} \in \mathrm{L} \cap \mathrm{L}^{\prime}$, and assume that $\ell_{a}=\ell_{i d x 1}$ and $\ell_{a}=\ell_{i d x 2}^{\prime \prime}$. Let $\sigma_{1}=\operatorname{apply}\left(\operatorname{apply}\left(\ldots \operatorname{apply}\left(\sigma_{0}, \arg \left(\ell_{1}\right)\right), \ldots, \arg (\right.\right.$ $\left.\left.\left.\left.\ell_{i d x 1-1}\right)\right) \cdot \arg \left(\ell_{i d x 1+1}\right)\right) \cdot \ldots \cdot \arg \left(\ell_{u}\right)\right)$ and $\sigma_{1}^{\prime \prime}=\operatorname{apply}\left(\operatorname{apply}\left(\ldots \operatorname{apply}\left(\sigma_{0}, \arg \left(\ell_{1}^{\prime \prime}\right)\right), \ldots, \arg (\right.\right.$ $\left.\left.\left.\left.\ell_{i d x 2-1}^{\prime \prime}\right)\right) \cdot \arg \left(\ell_{i d x 2+1}^{\prime \prime}\right)\right) \cdot \ldots \cdot \arg \left(\ell_{v}^{\prime \prime}\right)\right)$. By Prop ${ }_{1}^{\prime}$, we can see that $\sigma=\operatorname{apply}\left(\sigma_{1}, \arg \left(\ell_{i d x 1}\right)\right)$ and $\sigma^{\prime \prime}=\operatorname{apply}\left(\sigma_{1}^{\prime \prime}, \arg \left(\ell_{i d x 2}^{\prime \prime}\right)\right)$. By Prop $p_{3}^{\prime}$, we can see that $\sigma^{\prime}=\operatorname{merge}\left(\sigma, \sigma^{\prime \prime}\right)=\operatorname{merge}\left(\operatorname{apply}\left(\sigma_{1}\right.\right.$, $\left.\arg \left(\ell_{a}\right)\right)$, apply $\left.\left(\sigma_{1}^{\prime \prime}, \arg \left(\ell_{a}\right)\right)\right)=\operatorname{apply}\left(\operatorname{merge}\left(\sigma_{1}, \sigma_{1}^{\prime \prime}\right), \arg \left(\ell_{a}\right)\right)$. Assume that $\mathrm{L} \cap \mathrm{L}^{\prime \prime}=$ $\left\{x_{1}, \ldots, x_{l}\right\}, \mathrm{L} \backslash \mathrm{L}^{\prime \prime}=\left\{y_{1}, \ldots, y_{m}\right\}$ and $\mathrm{L}^{\prime \prime} \backslash \mathrm{L}=\left\{z_{1}, \ldots, z_{n}\right\}$. Then, by doing above process for several times, we can obtain that $\sigma^{\prime}=\operatorname{apply}\left(\operatorname{apply}\left(\ldots \operatorname{apply}\left(\operatorname{merge}\left(\sigma_{2}, \sigma_{2}^{\prime \prime}\right), \arg \left(x_{1}\right)\right), \ldots, \arg (\right.\right.$ $\left.\left.x_{l}\right)\right)$, where $\sigma_{2}=\operatorname{apply}\left(\operatorname{apply}\left(\ldots \operatorname{apply}\left(\sigma_{0}, \arg \left(y_{1}\right)\right), \ldots, \arg \left(y_{m}\right)\right)\right.$ and $\sigma_{2}^{\prime \prime}=\operatorname{apply}(\operatorname{apply}($ $\left.\ldots \operatorname{apply}\left(\sigma_{0}, \arg \left(z_{1}\right)\right), \ldots, \arg \left(z_{n}\right)\right)$.

Then, since $\left(L \backslash L^{\prime \prime}\right) \cap\left(L^{\prime \prime} \backslash L\right)=\emptyset$. and the visibility relation is transitive, we can see that for each method $m$, input value $a$, return value $b$ and replica $r^{\prime}$, it is impossible that both $\left(L \backslash L^{\prime \prime}\right)$ 
and $\left(\mathrm{L}^{\prime \prime} \backslash \mathrm{L}\right)$ contains operations that use method $m$, input value $a$, return value $b$ and originates in replica $r^{\prime}$. Therefore, the arguments of "local" effector of operations of $L \backslash L^{\prime \prime}$ is disjoint to the arguments of "local" effector of operations of $L$ " $\backslash L$.

Then, let us "move the local effectors of other operations out". By Prop $\sigma_{2}=\operatorname{apply}\left(\sigma_{5}, \arg \left(y_{1}\right)\right)$, where $\sigma_{5}=\operatorname{apply}\left(\operatorname{apply}\left(\ldots \operatorname{apply}\left(\sigma_{0}, \arg \left(y_{2}\right)\right), \ldots, \arg \left(y_{m}\right)\right)\right.$. We already know that the argument of "local" effector of $y_{1}$ is different than arguments of "local" effectors in $\left\{y_{2}, \ldots, y_{m}\right\} \cup\left\{z_{1}, \ldots, z_{n}\right\}$. Therefore, $P 2\left(\sigma_{5}, \arg \left(y_{1}\right)\right)$ and $P 2\left(\sigma_{2}^{\prime \prime}, \arg \left(y_{1}\right)\right)$ hold. Therefore, by Prop' and $\operatorname{Prop}_{4}$, we can see that merge $\left(\sigma_{2}, \sigma_{2}^{\prime \prime}\right)=\operatorname{merge}\left(\sigma_{2}^{\prime \prime}, \sigma_{2}\right)=$ $\operatorname{merge}\left(\sigma_{2}^{\prime \prime}, \operatorname{apply}\left(\sigma_{5}, \arg \left(y_{1}\right)\right)\right)=\operatorname{apply}\left(\operatorname{merge}\left(\sigma_{2}^{\prime \prime}, \sigma_{5}\right), \arg \left(y_{1}\right)\right)$. By doing this process for several times, we can prove that merge $\left(\sigma_{2}, \sigma_{2}^{\prime \prime}\right)=\operatorname{merge}\left(\sigma_{2}^{\prime \prime}, \sigma_{2}\right)=\operatorname{apply}(\operatorname{apply}(\ldots \operatorname{apply}($ merge $\left.\left.\left(\sigma_{2}^{\prime \prime}, \sigma_{0}\right), \arg \left(y_{m}\right)\right), \ldots, \arg \left(y_{1}\right)\right)$.

Similarly, we can prove that merge $\left(\sigma_{2}^{\prime \prime}, \sigma_{0}\right)=\operatorname{merge}\left(\sigma_{0}, \sigma_{2}^{\prime \prime}\right)=\operatorname{apply}(\operatorname{apply}(\ldots \operatorname{apply}(\operatorname{merge}($ $\left.\left.\left.\sigma_{0}, \sigma_{0}\right), \arg \left(z_{n}\right)\right), \ldots, \arg \left(z_{1}\right)\right)$. Therefore, by Prop ${ }_{1}^{\prime}$, we can see that, $\sigma^{\prime}$ can be obtained from merge $\left(\sigma_{0}, \sigma_{0}\right)$ by applying the "local" effectors of operations of $L \cup L^{\prime \prime}$ in any order. By Prop', we can see that, $\sigma^{\prime}$ can be obtained from $\sigma_{0}$ by applying the "local" effectors of operations of $L \cup L^{\prime \prime}$ in any order. Therefore, this lemma still holds for local configuration $\left(L^{\prime}, \sigma^{\prime}\right)$.

This completes the proof of this lemma.

Proving Refinement: Since our method has generator and "local" effector, similarly as Sec. 3, when the state-based CRDT object admits execution-order linearizations, we prove the existence of a refinement mapping by proving Refinement. Then, together with Lemma D.2, we can prove RA-linearizability.

\section{D.5 Proof Methodology for Idempotent Effectors}

In this subsection, we propose our proof methodology of RA-linearizability for the idempotent effectors.

A Lemma Similar to Lemma 4.2: We need to prove the following lemma, which states that $\sigma$ can be obtained from the initial replica state by applying "local" effectors of visible operations in any order. Especially, this holds when this order is the projection of linearization into visible operations. Here Prop 6 is a property that are introduced in the latter part of this subsection. It makes Lemma D.3 holds.

LEMMA D.3. Let o be a state-based CRDT that is idempotent effectors, and satisfies properties Prop $_{1}^{\prime}$, Prop $_{2}^{\prime}$, Prop $_{3}^{\prime}$, Prop $_{4}$, Prop $_{5}$ and Prop 6 . Given a history of o with a linearization of the operations in history(possibly, rewritten using a query-update rewriting $\gamma$ ), consistent with the visibility relation. Then, for each local configuration $(\mathrm{L}, \sigma)$ or message with content $(\mathrm{L}, \sigma)$,

$$
\sigma=\operatorname{apply}\left(\operatorname{apply}\left(\ldots \operatorname{apply}\left(\sigma_{0}, \arg \left(\ell_{1}\right)\right), \ldots, \arg \left(\ell_{k}\right)\right)\right.
$$

where $\sigma_{0}$ is the initial state and $\mathrm{L}=\left\{\ell_{1}, \ldots, \ell_{k}\right\}$. Especially, this holds when $\ell_{1} \cdot \ldots \cdot \ell_{k}=\operatorname{lin} \downarrow_{L}$.

Definition of Property Prop $_{6}$ : The definition of property Prop 6 is as follows:

- $\operatorname{Prop}_{6}$ : For each replica state $\sigma$ and operation $\ell$, apply $(\operatorname{apply}(\sigma, \arg (\ell)), \arg (\ell))=\operatorname{apply}(\sigma$, $\arg (\ell))$.

\section{Proof of Lemma D.3:}

PROOF. We prove by induction on executions. Obvious they hold in $\mathrm{gc}_{0}$. Assume they hold along the execution $\mathrm{gc}_{0} \rightarrow^{*} \mathrm{gc}$ and there is a new transition $\mathrm{gc} \rightarrow \mathrm{gc}^{\prime}$. We need to prove that they still hold in $\mathrm{gc}^{\prime}$. 
- For case when replica $r$ do operation $\ell$ : Same as that of Lemma D.2.

- For case when replica $r$ send a message: Same as that of Lemma D.2.

- For case when replica $r$ apply a message: Let $(L, \sigma)$ and $\left(L^{\prime}, \sigma^{\prime}\right)$ be the local configuration of replica $r$ of gc and gc', respectively. Let $\left(\mathrm{L}^{\prime \prime}, \sigma^{\prime \prime}\right)$ be the content of the message. We need to prove that this lemma still holds for local configuration $\left(\mathrm{L}^{\prime}, \sigma^{\prime}\right)$. By the semantics we have $\mathrm{L}^{\prime}=\mathrm{L} \cup \mathrm{L}^{\prime \prime}$ and $\sigma^{\prime}=\operatorname{merge}\left(\sigma, \sigma^{\prime \prime}\right)$.

By the induction assumption, we know that $\sigma=\operatorname{apply}\left(\operatorname{apply}\left(\ldots \operatorname{apply}\left(\sigma_{0}, \arg \left(\ell_{1}\right)\right), \ldots, \arg (\right.\right.$ $\left.\left.\ell_{u}\right)\right), \sigma^{\prime \prime}=\operatorname{apply}\left(\operatorname{apply}\left(\ldots \operatorname{apply}\left(\sigma_{0}, \arg \left(\ell_{1}^{\prime \prime}\right)\right), \ldots, \arg \left(\ell_{v}^{\prime \prime}\right)\right)\right.$, where $\mathrm{L}=\left\{\ell_{1}, \ldots, \ell_{u}\right\}$ and $\mathrm{L}^{\prime \prime}=\left\{\ell_{1}^{\prime \prime}, \ldots, \ell_{v}^{\prime \prime}\right\}$.

Let us "move the local effectors of duplicate operations out". If $\mathrm{L} \cap \mathrm{L}$ " $\neq \emptyset$ : Let $\ell_{a} \in \mathrm{L} \cap \mathrm{L}^{\prime}$, and assume that $\ell_{a}=\ell_{i d x 1}$ and $\ell_{a}=\ell_{i d x 2}^{\prime \prime}$. Let $\sigma_{1}=\operatorname{apply}\left(\operatorname{apply}\left(\ldots \operatorname{apply}\left(\sigma_{0}, \arg \left(\ell_{1}\right)\right), \ldots, \arg (\right.\right.$ $\left.\left.\left.\left.\ell_{i d x 1-1}\right)\right) \cdot \arg \left(\ell_{i d x 1+1}\right)\right) \cdot \ldots \cdot \arg \left(\ell_{u}\right)\right)$ and $\sigma_{1}^{\prime \prime}=\operatorname{apply}\left(\operatorname{apply}\left(\ldots \operatorname{apply}\left(\sigma_{0}, \arg \left(\ell_{1}^{\prime \prime}\right)\right), \ldots, \arg (\right.\right.$ $\left.\left.\left.\left.\ell_{i d x_{2-1}}^{\prime \prime}\right)\right) \cdot \arg \left(\ell_{i d x 2+1}^{\prime \prime}\right)\right) \cdot \ldots \cdot \arg \left(\ell_{v}^{\prime \prime}\right)\right)$. By $\operatorname{Prop}_{1}^{\prime}$, we can see that $\sigma=\operatorname{apply}\left(\sigma_{1}, \arg \left(\ell_{i d x 1}\right)\right)$ and $\sigma^{\prime \prime}=\operatorname{apply}\left(\sigma_{1}^{\prime \prime}, \arg \left(\ell_{i d x 2}^{\prime \prime}\right)\right)$. By Prop ${ }_{3}^{\prime}$, we can see that $\sigma^{\prime}=\operatorname{merge}\left(\sigma, \sigma^{\prime \prime}\right)=\operatorname{merge}\left(\operatorname{apply}\left(\sigma_{1}\right.\right.$, $\left.\arg \left(\ell_{a}\right)\right)$, apply $\left.\left(\sigma_{1}^{\prime \prime}, \arg \left(\ell_{a}\right)\right)\right)=\operatorname{apply}\left(\operatorname{merge}\left(\sigma_{1}, \sigma_{1}^{\prime \prime}\right), \arg \left(\ell_{a}\right)\right)$. Assume that $\mathrm{L} \cap \mathrm{L}^{\prime \prime}=$ $\left\{x_{1}, \ldots, x_{l}\right\}, \mathrm{L} \backslash \mathrm{L}^{\prime \prime}=\left\{y_{1}, \ldots, y_{m}\right\}$ and $\mathrm{L}^{\prime \prime} \backslash \mathrm{L}=\left\{z_{1}, \ldots, z_{n}\right\}$. Then, by doing above process for several times, we can obtain that $\sigma^{\prime}=\operatorname{apply}\left(\operatorname{apply}\left(\ldots \operatorname{apply}\left(\operatorname{merge}\left(\sigma_{2}, \sigma_{2}^{\prime \prime}\right), \arg \left(x_{1}\right)\right), \ldots, \arg (\right.\right.$ $\left.\left.x_{l}\right)\right)$, where $\sigma_{2}=\operatorname{apply}\left(\operatorname{apply}\left(\ldots \operatorname{apply}\left(\sigma_{0}, \arg \left(y_{1}\right)\right), \ldots, \arg \left(y_{m}\right)\right)\right.$ and $\sigma_{2}^{\prime \prime}=\operatorname{apply}(\operatorname{apply}($ $\left.\ldots \operatorname{apply}\left(\sigma_{0}, \arg \left(z_{1}\right)\right), \ldots, \arg \left(z_{n}\right)\right)$.

Then, Let us "move the local effectors of duplicate arguments out".

If there exists operation $\ell_{b} \in \mathrm{L} \backslash \mathrm{L}^{\prime \prime}$ and operation $\ell_{c} \in \mathrm{L}^{\prime \prime} \backslash \mathrm{L}$, such that they use a same method, same input value and same return value, and thus, have same argument of "local" effector: Assume that $\ell_{b}=y_{i d x b}$ and $\ell_{c}=z_{i d x c}$. Let $\sigma_{3}=\operatorname{apply}\left(\operatorname{apply}\left(\ldots \operatorname{apply}\left(\sigma_{0}, \arg \left(y_{1}\right)\right), \ldots, \arg (\right.\right.$ $\left.\left.\left.\left.y_{i d x b-1}\right)\right) \cdot \arg \left(y_{i d x b+1}\right)\right) \cdot \ldots \cdot \arg \left(y_{m}\right)\right)$ and $\sigma_{3}^{\prime \prime}=\operatorname{apply}\left(\operatorname{apply}\left(\ldots \operatorname{apply}\left(\sigma_{0}, \arg \left(z_{1}\right)\right), \ldots, \arg (\right.\right.$ $\left.\left.\left.\left.z_{i d x c-1}\right)\right) \cdot \arg \left(z_{i d x c+1}\right)\right) \cdot \ldots \cdot \arg \left(z_{n}\right)\right)$. By Prop ${ }_{1}^{\prime}$, we can see that $\sigma_{2}=\operatorname{apply}\left(\sigma_{3}, \arg \left(y_{i d x b}\right)\right)$ and $\sigma_{2}^{\prime \prime}=\operatorname{apply}\left(\sigma_{3}^{\prime \prime}, \arg \left(z_{i d x c}\right)\right)$. By Prop ${ }_{3}^{\prime}$ and $\operatorname{Prop}_{6}$, we can see that merge $\left(\sigma_{2}, \sigma_{2}^{\prime \prime}\right)=$ $\operatorname{merge}\left(\operatorname{apply}\left(\sigma_{3}, \arg \left(y_{i d x b}\right)\right), \operatorname{apply}\left(\sigma_{3}^{\prime \prime}, \arg \left(z_{i d x c}\right)\right)\right)=\operatorname{apply}\left(\operatorname{merge}\left(\sigma_{3}, \sigma_{3}^{\prime \prime}\right), \arg \left(y_{i d x b}\right)\right)=$ $\operatorname{apply}\left(\operatorname{apply}\left(\operatorname{merge}\left(\sigma_{3}, \sigma_{3}^{\prime \prime}\right), \arg \left(y_{i d x b}\right)\right), \arg \left(z_{\text {dixc }}\right)\right)$.

Let $S_{1}=\left\{\ell \mid \ell \in \mathrm{L} \backslash \mathrm{L}^{\prime \prime}, \mathrm{L}^{\prime \prime} \backslash \mathrm{L}\right.$ contains an operation with same method, same input value and same return value of $\ell\} \cup\left\{\ell \mid \ell \in \mathrm{L}^{\prime \prime} \backslash \mathrm{L}, \mathrm{L} \backslash \mathrm{L}^{\prime \prime}\right.$ contains an operation with same method, same input value and same return value of $\ell\}$. Assume that $S_{1} \cup\left(\mathrm{L} \cap \mathrm{L}^{\prime \prime}\right)=\left\{o_{1}, \ldots, o_{r}\right\}$, $\left(\mathrm{L} \backslash \mathrm{L}^{\prime \prime}\right) \backslash S_{1}=\left\{p_{1}, \ldots, p_{s}\right\}$ and $\left(\mathrm{L}^{\prime \prime} \backslash \mathrm{L}\right) \backslash S_{1}=\left\{q_{1}, \ldots, q_{t}\right\}$. By doing this process for several times, we can obtain that $\sigma^{\prime}=\operatorname{apply}\left(\operatorname{apply}\left(\ldots \operatorname{apply}\left(\operatorname{merge}\left(\sigma_{4}, \sigma_{4}^{\prime \prime}\right), \arg \left(o_{1}\right)\right), \ldots\right.\right.$, $\left.\arg \left(o_{r}\right)\right)$, where $\sigma_{4}=\operatorname{apply}\left(\operatorname{apply}\left(\ldots \operatorname{apply}\left(\sigma_{0}, \arg \left(p_{1}\right)\right), \ldots, \arg \left(p_{s}\right)\right)\right.$ and $\sigma_{4}^{\prime \prime}=\operatorname{apply}($ $\operatorname{apply}\left(\ldots \operatorname{apply}\left(\sigma_{0}, \arg \left(q_{1}\right)\right), \ldots, \arg \left(q_{t}\right)\right)$. Moreover, we can see that, the arguments of "local" effector of operations of $\left(\mathrm{L} \backslash \mathrm{L}^{\prime \prime}\right) \backslash S_{1}$ is disjoint to the arguments of "local" effector of operations of $\left(\mathrm{L}^{\prime \prime} \backslash \mathrm{L}\right) \backslash S_{1}$.

Then, let us "move the local effectors of other operations out". By Prop', we can see that $\sigma_{4}=\operatorname{apply}\left(\sigma_{6}, \arg \left(p_{1}\right)\right)$, where $\sigma_{6}=\operatorname{apply}\left(\operatorname{apply}\left(\ldots \operatorname{apply}\left(\sigma_{0}, \arg \left(p_{2}\right)\right), \ldots, \arg \left(p_{s}\right)\right)\right.$. We already know that the argument of "local" effector of $p_{1}$ is different than arguments of "local" effectors in $\left\{p_{2}, \ldots, p_{s}\right\} \cup\left\{q_{1}, \ldots, q_{t}\right\}$. Therefore, $P 2\left(\sigma_{6}, \arg \left(p_{1}\right)\right)$ and $P 2\left(\sigma_{4}^{\prime \prime}, \arg \left(p_{1}\right)\right)$ hold. Therefore, by Prop $p_{2}^{\prime}$ and $\operatorname{Prop}_{4}$, we can see that merge $\left(\sigma_{4}, \sigma_{4}^{\prime \prime}\right)=\operatorname{merge}\left(\sigma_{4}^{\prime \prime}, \sigma_{4}\right)=$ $\operatorname{merge}\left(\sigma_{4}^{\prime \prime}, \operatorname{apply}\left(\sigma_{6}, \arg \left(p_{1}\right)\right)\right)=\operatorname{apply}\left(\operatorname{merge}\left(\sigma_{4}^{\prime \prime}, \sigma_{6}\right), \arg \left(p_{1}\right)\right)$. By doing this process for several times, we can prove that $\operatorname{merge}\left(\sigma_{4}, \sigma_{4}^{\prime \prime}\right)=\operatorname{merge}\left(\sigma_{4}^{\prime \prime}, \sigma_{4}\right)=\operatorname{apply}(\operatorname{apply}(\ldots \operatorname{apply}($ $\left.\left.\operatorname{merge}\left(\sigma_{4}^{\prime \prime}, \sigma_{0}\right), \arg \left(p_{s}\right)\right), \ldots, \arg \left(p_{1}\right)\right)$. Similarly, we can prove that merge $\left(\sigma_{4}^{\prime \prime}, \sigma_{0}\right)=\operatorname{merge}\left(\sigma_{0}\right.$, $\left.\sigma_{4}^{\prime \prime}\right)=\operatorname{apply}\left(\operatorname{apply}\left(\ldots \operatorname{apply}\left(\operatorname{merge}\left(\sigma_{0}, \sigma_{0}\right), \arg \left(q_{t}\right)\right), \ldots, \arg \left(q_{1}\right)\right)\right.$. Therefore, by Prop ${ }_{1}^{\prime}$, we can see that, $\sigma^{\prime}$ can be obtained from merge $\left(\sigma_{0}, \sigma_{0}\right)$ by applying the "local" effectors of 
operations of $L \cup L^{\prime \prime}$ in any order. By Prop $p_{4}^{\prime}$, we can see that, $\sigma^{\prime}$ can be obtained from $\sigma_{0}$ by applying the "local" effectors of operations of $L \cup L^{\prime}$ " in any order. Therefore, this lemma still holds for local configuration $\left(\mathrm{L}^{\prime}, \sigma^{\prime}\right)$ in the case of $C_{2}$.

This completes the proof of this lemma.

Proving Refinement: Since our method has generator and "local" effector, similarly as Sec. 3, when the state-based CRDT object admits execution-order linearizations, we prove the existence of a refinement mapping by proving Refinement. Then, together with Lemma D.3, we can prove RA-linearizability.

\section{E IMPLEMENTATIONS OF STATE-BASED CRDT AND THEIR SEQUENTIAL SPECIFICATIONS}

Let us introduce state-based CRDT implementations, their sequential specifications, and their "local" effectors and arguments of "local" effectors.

\section{E.1 State-Based Multi-Value Register, Its Sequential Specification and Its "Local" Effectors}

Implementation: The state-based multi-value register implementation of [Shapiro et al. 2011] is given in Listing 7. It implements an interface with operations: write $(a)$ and read. This implementation assumes that the number of replicas are fixed. A payload $S$ is a set of $(a, V)$ pairs, where $a$ is a value and $V$ is a vector called version vector. The size of $V$ is the number of replicas in distributed system. myRep() is a function that returns current replica identifier. A partial order among version vectors are defined as: Given version vectors $V$ and $V^{\prime}$, we say that $V>V^{\prime}$, if for each replica $r$, we have $V[r] \geq V^{\prime}[r]$, and there exists replica $r^{\prime}$, such that $V\left[r^{\prime}\right]>V^{\prime}\left[r^{\prime}\right]$.

write $(a)$ generates a new version vector $V^{\prime}$, and set the payload into $\left\{\left(a, V^{\prime}\right)\right\}$. read returns the set of values of multi-value register.

The query-update rewriting rewrites write(a) into write $\left(a, V^{\prime}\right)$, where $V^{\prime}$ is the version vector generated by executing write(a).

Sequential Specification Spec(MV-Reg): Each abstract state $\phi$ is a set of tuples $(a, i d)$, where $a$ is a data and $i d$ is an identifier. Moreover, we assume that there is a partial order on identifier. The sequential specification Spec(MV-Reg) of multi-value register is defined by:

$$
\begin{aligned}
(\phi \mid \text { id is not less or equal than any identifier of } \phi) & \stackrel{\text { write(a, id })}{\longrightarrow} \phi \cup\{(a, \text { id })\} \backslash\left\{\left(a^{\prime}, i d^{\prime}\right) \in \phi, i d^{\prime}<i d\right\} \\
\phi & \stackrel{\operatorname{read}() \Rightarrow S}{\longrightarrow} \phi[\text { with } S=\{a \mid \exists \text { id, }(a, \text { id }) \in \phi\}]
\end{aligned}
$$

Method write $(a, i d)$ puts $\{(a, i d)\}$ into the abstract state and then removes from the abstract state all pairs with a less identifier. Method read returns the value of abstract state.

The "Local" Effector, Arguments, Partial Order, and Predicate $P 1$ : The state-based multi-value register is uniquely-identified-effectors. Given operation $\ell=$ write $(a)$, which generates version vector $V$, then, $\arg (\ell)=(a, V)$. The partial order of arguments of "local" effector is defined as follows: $\left(a_{1}, V_{1}\right)$ is less than $\left(a_{2}, V_{2}\right)$, if $V_{1}<V_{2}$. The apply method is defined as follows: Given $\sigma=S$ and $\arg (\ell)=(a, V) . \operatorname{apply}(\sigma, \arg (\ell))=S \cup\{(a, V)\} \backslash\left\{\left(a^{\prime}, V^{\prime}\right) \mid\left(a^{\prime}, V^{\prime}\right) \in S, V^{\prime}<V\right\}$. Predicate $P 1$ is defined as follows: $P 1(\sigma,(a, V))$ holds, if for each $\left(a^{\prime}, V^{\prime}\right) \in \sigma, V$ is not less than $V^{\prime}$.

Properties of Version Vectors: The follow lemma states that the version vector of argument of "local" effector of each write operation is unique, and the order between version vectors is consistent with the visibility relation. 


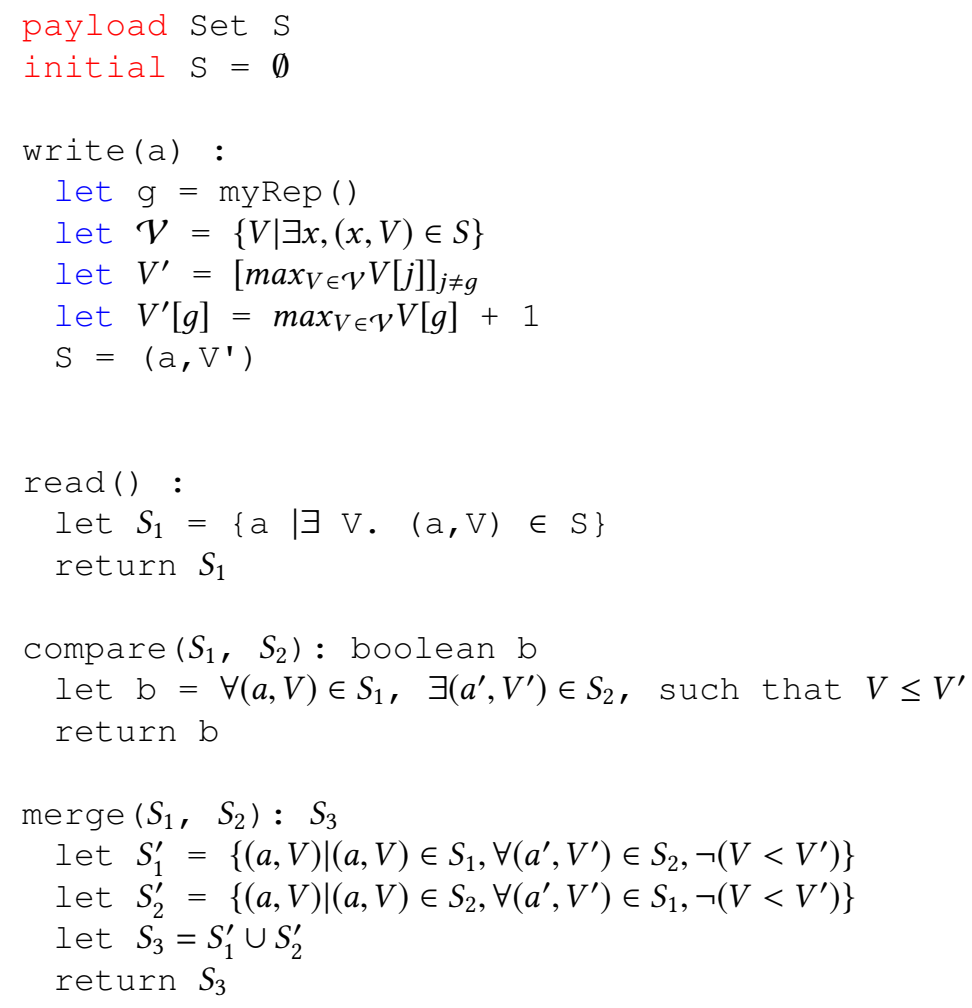

Listing 7. Pseudo-code of state-based multi-value register

LEMMA E.1. The version vector is unique for each write operation, and the order of version vector is consistent with the visibility relation.

The follow lemma states that version vectors of concurrent write operations are incomparable.

LEMMA E.2. Given two write operation $\ell_{1}$ and $\ell_{2}$, assume $\left(a_{1}, V_{1}\right)$ and $\left(a_{2}, V_{2}\right)$ is the argument of "local" effector for $\ell_{1}$ and $\ell_{2}$, respectively, and assume that $\ell_{1}$ and $\ell_{2}$ are concurrent. Then, $\neg\left(V_{1}<V_{2} \vee V_{2}<V_{1}\right)$.

Proof of Lemma E.1:

ProOF. Let us propose $f$ act 1, fact 2 , fact 3 and fact 4 :

- fact1: Given two write operation $\ell_{1}$ and $\ell_{2}$, assume $\left(a_{1}, V_{1}\right)$ and $\left(a_{2}, V_{2}\right)$ is the argument of "local" effector for $\ell_{1}$ and $\ell_{2}$, respectively, and assume that $\left(\ell_{1}, \ell_{2}\right) \in$ vis. Then, $V_{1}<V_{2}$.

- fact2: Given two write operation $\ell_{1}$ and $\ell_{2}$, assume $\left(a_{1}, V_{1}\right)$ and $\left(a_{2}, V_{2}\right)$ is the argument of "local" effector for $\ell_{1}$ and $\ell_{2}$, respectively, and assume that $\ell_{1}$ and $\ell_{2}$ are concurrent. Then, $\neg\left(V_{1}<V_{2} \vee V_{2}<V_{1}\right)$.

- fact3: Assume $(a, V)$ is the argument of "local" effector of a write(a) operation $\ell$. Then, for each replica $r, V[r]=\mid\left\{\ell^{\prime}=\right.$ write(_), $\ell^{\prime}$ originate in replica $r,\left(\ell^{\prime}, \ell\right) \in$ vis $\left.\vee \ell^{\prime}=\ell\right\} \mid$.

- fact4: Assume $(\mathrm{L}, S)$ is the local configuration of a replica or the content of a message. Then, $S=\{(a, V) \mid \exists \ell,(a, V)$ is the argument of "local" effector of $\ell, \ell$ is maximal w.r.t vis among write operations in $\mathrm{L}\}$. 
Assume that above four facts are proved. Then, since the visibility relation is a partial order, by fact 3 we can see that the version vector is unique for each write operation, and by fact 1 we can see that the order of version vector is consistent with the visibility relation.

Let us prove fact1. Given two write operation $\ell_{1}$ and $\ell_{2}$, assume $\left(a_{1}, V_{1}\right)$ and $\left(a_{2}, V_{2}\right)$ is the argument of "local" effector for $\ell_{1}$ and $\ell_{2}$, respectively, and assume that $\left(\ell_{1}, \ell_{2}\right) \in$ vis. Then, there are two possibilities.

- If $\ell_{1}$ and $\ell_{2}$ originate in a same replica, then fact 1 obviously holds according to the implementation.

- Otherwise, there exists messages $\left(L_{1}, \sigma_{1}\right), \ldots,\left(L_{k}, \sigma_{k}\right)$ and replica $r_{1}, \ldots, r_{k+1}$, such that (1) $\ell_{1}$ originates in replica $r_{1}, \ell_{2}$ originates in replica $r_{k+1},(2)$ for each $1 \leq i \leq k$, message $\left(L_{i}, \sigma_{i}\right)$ is generated by replica $r_{i}$, (3) the time point of originating $\ell_{1}$ is earlier than the time point of generating message $\left(\mathrm{L}_{1}, \sigma_{1}\right)$, (4) for each $1 \leq i \leq k$, message $\left(\mathrm{L}_{i}, \sigma_{i}\right)$ is received by replica $r_{i+1}$, and such time point is earlier than the time point of generating message $\left(\mathrm{L}_{i+1}, \sigma_{i+1}\right)$, and (5) the time point of receiving message $\left(\mathrm{L}_{k}, \sigma_{k}\right)$ by replica $r_{k+1}$ is earlier than the time point of originating $\ell_{2}$.

According to the implementation, we can see that for each message $\left(\mathrm{L}_{i}, \sigma_{i}\right)$ with $1 \leq i \leq k$, there exists $\left(a_{i}^{\prime}, i d_{i}^{\prime}\right) \in \sigma_{i}$, such that $V_{1} \leq i d_{i}^{\prime}$. According to the implementation, for each $\left(a^{\prime}, i d^{\prime}\right) \in \sigma_{k}$, we have $i d^{\prime}<V_{2}$. Therefore, we can see that $f a c t 1$ still holds.

This completes the proof of fact 1 .

Let us prove that fact 2 , fact 3 and fact 4 are inductive invariant. We prove by induction on executions. Obvious they hold in $\mathrm{gc}_{0}$. Assume they hold along the execution $\mathrm{gc}_{0} \rightarrow^{*} \mathrm{gc}$ and there is a new transition $\mathrm{gc} \rightarrow \mathrm{gc}^{\prime}$.

- For case when replica $r$ do write(a): let $\left(a, V^{\prime}\right)$ be the argument of "local" effector of $\ell$. Let $L c=(\mathrm{L}, S)$ and $L c^{\prime}=\left(\mathrm{L}^{\prime}, S^{\prime}\right)$ be the local configuration of replica $\mathrm{r}$ of gc and $g \mathrm{c}^{\prime}$, respectively. Obviously $S^{\prime}=\left\{\left(a, V^{\prime}\right)\right\}$ and $\mathrm{L}^{\prime}=\mathrm{L} \cup\{\ell\}$. We need to prove that $f$ act 4 still holds for the local configuration $L c^{\prime}$, fact 3 still holds for the argument of "local" effector of $\ell$, and fact 2 still holds in $\mathrm{gc}^{\prime}$.

Since $\ell$ is greater than any operations of $L$ w.r.t the visibility relation, fact 4 still holds for the local configuration $L c^{\prime}$.

Let $\mathcal{V}=\{V \mid(,, V) \in S\}$ be the set of version vectors of $S$. By fact 4 of local configuration $L c$, fact 3 of arguments of "local" effectors of $S$, and the transitivity of the visibility relation, we can see that, for each replica $r^{\prime}, \max _{V_{1} \in \mathcal{V}} V_{1}\left[r^{\prime}\right]$ is the number of write operations originates in replica $r^{\prime}$ and is in L. It is obvious that, for each replica $r^{\prime} \neq r, V^{\prime}\left[r^{\prime}\right]=\max _{V_{1} \in \mathcal{V}} V_{1}\left[r^{\prime}\right]$, and

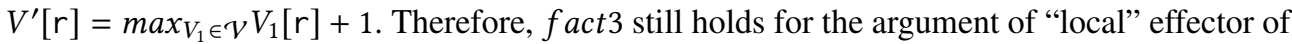
$\ell$.

We prove $f a c t 2$ by contradiction. Since fact 2 holds in gc, it is easy to see that the counterexample of fact 2 must contain operation $\ell$. Assume there exists an operation $\ell_{2}$ that is

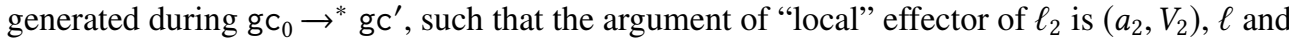
$\ell_{2}$ are concurrent, and $V^{\prime}<V_{2}$. We can see that $V^{\prime}[r] \leq V_{2}[r]$. By fact 3 of the arguments of "local" effectors $\left(a, V^{\prime}\right)$ and $\left(a_{2}, V_{2}\right)$, and the transitivity of the visibility relation, we can see that $\left(\ell, \ell_{2}\right) \in$ vis, which contradicts the assumption that $\ell$ and $\ell_{2}$ are concurrent.

Similarly, assume there exists an operation $\ell_{2}$ that is generated during $\mathrm{gc}_{0} \rightarrow^{*} \mathrm{gc}^{\prime}$, such that the argument of "local" effector of $\ell_{2}$ is $\left(a_{2}, V_{2}\right), \ell$ and $\ell_{2}$ are concurrent, and $V_{2}<V^{\prime}$. Assume that $\ell_{2}$ is originated in replica $r_{2}$. We can see that $V_{2}\left[r_{2}\right] \leq V^{\prime}\left[r_{2}\right]$. By fact 3 of the arguments of "local" effectors $\left(a, V^{\prime}\right)$ and $\left(a_{2}, V_{2}\right)$, and the transitivity of the visibility relation, we can see that $\left(\ell_{2}, \ell\right) \in$ vis, which contradicts the assumption that $\ell$ and $\ell_{2}$ are concurrent. Therefore, fact2 still holds in $\mathrm{gc}^{\prime}$. 
- For case when replica $r$ generate a message: Let $L c=(\mathrm{L}, S)$ and $L c^{\prime}=\left(\mathrm{L}^{\prime}, S^{\prime}\right)$ be the local configuration of replica $r$ of gc and gc $c^{\prime}$, respectively. By the semantics we have $\left(\mathrm{L}^{\prime}, S^{\prime}\right)=(\mathrm{L}, S)$ and the content of the message is $(\mathrm{L}, S)$. We only need to prove that fact4 still holds for the message (L, $S$ ). This holds obviously since by induction assumption we already knows that fact 4 holds for the local configuration $(\mathrm{L}, S)$.

- For case when replica $r$ apply a message: Let $L c=(\mathrm{L}, S)$ and $L c^{\prime}=\left(\mathrm{L}^{\prime}, S^{\prime}\right)$ be the local configuration of replica $r$ of gc and $g^{\prime}$, respectively. Assume that the message content is $\left(\mathrm{L}^{\prime \prime}, S^{\prime \prime}\right)$. By the semantics we have $\mathrm{L}^{\prime}=\mathrm{L} \cup \mathrm{L}^{\prime \prime}$ and $S^{\prime}=\operatorname{merge}\left(\mathrm{S}, \mathrm{S}^{\prime \prime}\right)$. We need to prove that fact4 still holds for the local configuration $\left(\mathrm{L}^{\prime}, S^{\prime}\right)$.

Let us prove by contradiction. Assume that fact 4 does not holds for the local configuration $\left(\mathrm{L}^{\prime}, S^{\prime}\right)$.

It is easy to see that, the arguments of "local" effectors of maximum of write operation w.r.t. vis in $L^{\prime}$ must be in that of $L$ or $L^{\prime \prime}$. Then, there are two possibilities:

- There exists a argument of "local" effector $\left(a_{1}, V_{1}\right) \in S$, such that $\left(a_{1}, V_{1}\right) \in S^{\prime}$, and there exists $\left(a_{2}, V_{2}\right) \in S^{\prime}$, such that $\left(a_{1}, V_{1}\right)$ and $\left(a_{2}, V_{2}\right)$ is the argument of "local" effector of some operation $\ell_{1}$ and $\ell_{2}$, respectively, and $\left(\ell_{1}, \ell_{2}\right) \in$ vis. By fact 1 and fact 2 , we can see that $V_{1}<V_{2}$.

By fact 1, fact 2 , fact 3 and fact 4 , we can see that the version vectors of $S$ are pair-wise incomparable, and the version vectors of $S^{\prime \prime}$ are pair-wise incomparable. Then, we can see that $\left(a_{1}, V_{1}\right) \in S \wedge\left(a_{2}, V_{2}\right) \in S^{\prime \prime}$. According to the merge method, it is easy to see that $\left(a_{1}, V_{1}\right) \notin S^{\prime}$, which contradicts that $\left(a_{1}, V_{1}\right) \in S^{\prime}$.

For the case when there exists a local effector $\left(a_{1}, V_{1}\right) \in S^{\prime \prime}$, such that $\left(a_{1}, V_{1}\right) \in S^{\prime}$, and there exists $\left(a_{2}, V_{2}\right) \in S^{\prime}$, such that $\left(a_{1}, V_{1}\right)$ and $\left(a_{2}, V_{2}\right)$ is the argument of "local" effector of some operation $\ell_{1}$ and $\ell_{2}$, respectively, and $\left(\ell_{1}, \ell_{2}\right) \in$ vis. Similarly, we can see that $\left(a_{1}, V_{1}\right) \in S^{\prime \prime} \wedge\left(a_{2}, V_{2}\right) \in S$. According to the merge method, it is easy to see that $\left(a_{1}, V_{1}\right) \notin S^{\prime}$, which contradicts that $\left(a_{1}, V_{1}\right) \in S^{\prime}$.

- There exists an operation $\ell_{1}$ with its argument $\left(a_{1}, V_{1}\right)$ of "local" effector, such that $\ell_{1}$ is maximal of write operation w.r.t. vis in $\mathrm{L}^{\prime}$, and $\left(a_{1}, V_{1}\right) \notin S^{\prime}$. Then, there must exists an operation $\ell_{2}$ with its argument $\left(a_{2}, V_{2}\right)$ of "local" effector, such that either $\left(a_{1}, V_{1}\right) \in$ $S \wedge\left(a_{2}, V_{2}\right) \in S^{\prime \prime} \wedge V_{1}<V_{2}$, or $\left(a_{1}, V_{1}\right) \in S^{\prime \prime} \wedge\left(a_{2}, V_{2}\right) \in S \wedge V_{1}<V_{2}$. By fact 1 and fact 2 , in each case, $\ell_{1}$ is not maximal of write operation w.r.t. vis in $L^{\prime}$, which is a contradiction. Therefore, fact 4 still holds for the local configuration $\left(\mathrm{L}^{\prime}, S^{\prime}\right)$.

This completes the proof of this lemma.

Proof of Lemma E.2: Obviously from the proof of Lemma E.1.

\section{E.2 State-Based Last-Writer-Win-Element-Set (LWW-Element-Set), Its Sequential Specification and Its "Local" Effectors}

Implementation: The state-based last-writer-win-element-set (LWW-element-set) of [Shapiro et al. 2011] is given in Listing 8. It implements a set interface with operations: $\operatorname{add}(a), \operatorname{remove}(a)$ and read. A payload $(A, R)$ contains a set $A$ that records pairs of inserted values and their timestamp, and a set $R$ that records the pairs of removed values and and their timestamp, and $R$ is used as tombstone.

A value $b$ is "in the set", if $\left(b, t s_{b}\right) \in A$ for some timestamp $t s_{b}$, and there does not exists $\left(b, t s_{b}^{\prime}\right) \in R$, such that $t s_{b}<t s_{b}^{\prime}$. write $(a)$. write $(a)$ generates a new timestamp $t s$ and puts $(a, t s)$ into set $A$. remove $(a)$ generates a new timestamp $t s$ and puts $(a, t s)$ into set $R$. read returns the set content. 


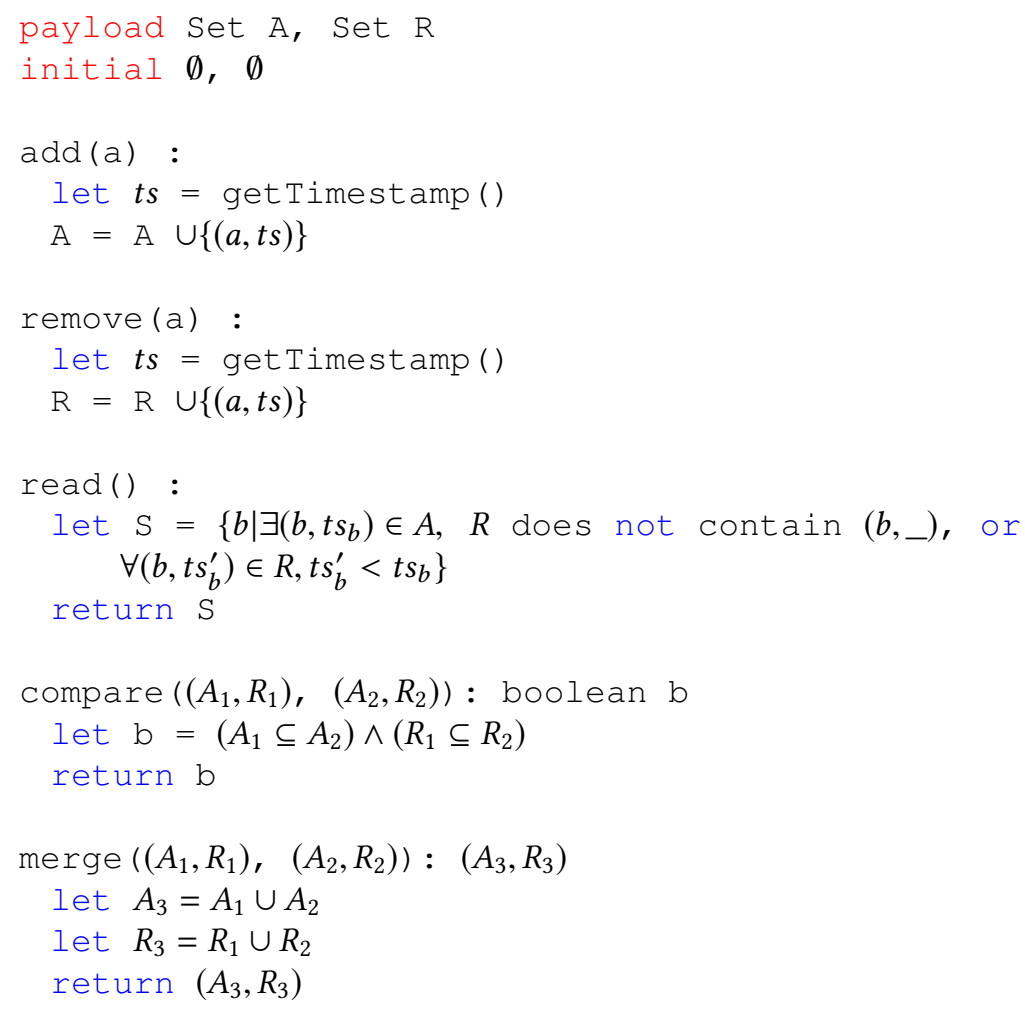

Listing 8. Pseudo-code of state-based LWW-element-set

Sequential Specification $\operatorname{Spec}($ Set): Each abstract state $\phi$ is a set $S$ of values. The sequential specification $\operatorname{Spec}(\operatorname{Set})$ of set is defined by:

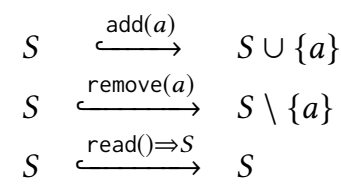

Method add(a) puts $a$ into $S$. Method remove(a) removes $a$ from $S$. Method $\operatorname{read}() \Rightarrow S$ returns the contents of the set.

The "Local" Effector, Arguments, Partial Order, and Predicate $P 1$ : The state-based LWWelement-set is uniquely-identified-effectors. Given operation $\ell=\operatorname{add}(\mathrm{a}) \stackrel{\mathrm{ts} a}{\Rightarrow}$, then, $\arg (\ell)=$ $\left(a d d, a, t s_{a}\right)$; given operation $\ell=\operatorname{remove}(a) \stackrel{\mathrm{ts}_{a}}{\Rightarrow}, \operatorname{then}, \arg (\ell)=\left(\right.$ rem, $\left.a, t s_{a}\right)$. The partial order of arguments of "local" effector is defined as follows: $\left({ }_{-}, t, t s\right)$ is less than $\left(_{-},+, t s^{\prime}\right)$, if $t s<$ $t s^{\prime}$. The apply method is defined as follows: Given $\sigma=(A, R)$ and $\arg (\ell)=\left(a d d, a, t s_{a}\right)$, $\operatorname{apply}(\sigma, \arg (\ell))=\left(A \cup\left\{\left(a, t s_{a}\right)\right\}, R\right)$. Given $\sigma=(A, R) \operatorname{and} \arg (\ell)=\left(\operatorname{rem}, a, t s_{a}\right), \operatorname{apply}(\sigma, \arg (\ell))=$ $\left(A, R \cup\left\{\left(a, t s_{a}\right)\right\}\right)$. Predicate $P 1$ is defined as follows: $P 1\left((A, R),\left({ }_{-}, t s\right)\right)$ holds, if for each $\left(\_, t s^{\prime}\right) \in$ $A \cup R, t s$ is not less than $t s^{\prime}$. 


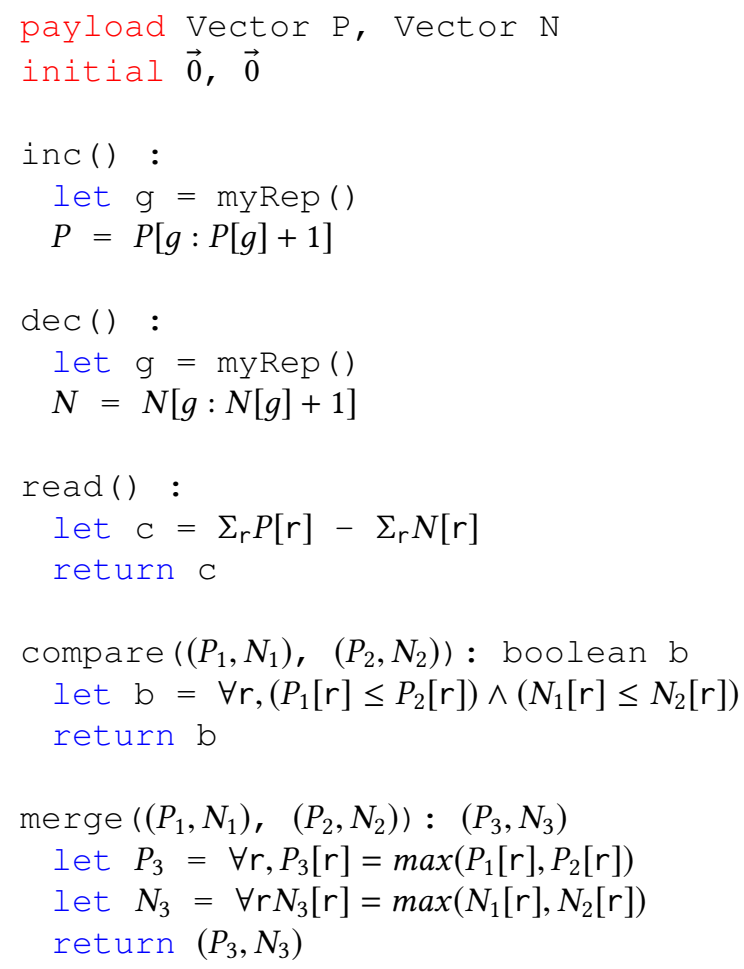

Listing 9. Pseudo-code of state-based PN-counter

\section{E.3 State-Based PN-Counter, Its Sequential Specification and Its "Local” Effectors}

Implementation: The state-based PN-counter implementation of [Shapiro et al. 2011] is given in Listing 9. It implements a counter interface with operations: inc(), dec( $)$ and read. This implementation assumes that the number of replicas are fixed. A payload $(P, N)$ contains a vector $P$ and a vector $N$. The size of $P$ and $N$ is the number of replicas in distributed system. myRep() is a function that returns current replica identifier. Let us use $\overrightarrow{0}$ to indicate the vector that maps each replica identifier to 0 , and the initial value of $P$ and $N$ is $\overrightarrow{0}$.

The counter value is $\Sigma_{r} P[r]-\Sigma_{r} N[r]$. inc() that originates in replica $r$ increases $P[r]$ by $1 . \operatorname{dec}()$ that originates in replica $r$ increases $N[r]$ by 1 . read returns the counter value.

Sequential Specification: PN-counter uses the sequential specification Spec(Counter).

The "Local" Effector, Arguments, and Predicate P2: The state-based PN-counter is cumulative effectors. Given operation $\ell=$ inc() that originates in replica $r$, then, $\arg (\ell)=($ inc, $r$ ); given operation $\ell=\operatorname{dec}()$ that originates in replica $r$, then, $\arg (\ell)=(\operatorname{dec}, r)$. The apply method is defined as follows: Given $\sigma=(P, N)$ and $\arg (\ell)=($ inc, $r)$, apply $(\sigma, \arg (\ell))=(P[r \leftarrow P[r]+1], N)$. Given $\sigma=(P, N)$ and $\arg (\ell)=(d e c, r), \operatorname{apply}(\sigma, \arg (\ell))=(P, N[r \leftarrow N[r]+1])$. Predicate $P 2$ is defined as follows: Given $\sigma=(P, N)$ and $\arg (\ell)=($ inc, $r), P 2(\sigma, \arg (\ell))$ holds, if $P[r]=0$; Given $\sigma=(P, N)$ and $\arg (\ell)=(\operatorname{dec}, r), P 2(\sigma, \arg (\ell))$ holds, if $N[r]=0$. 


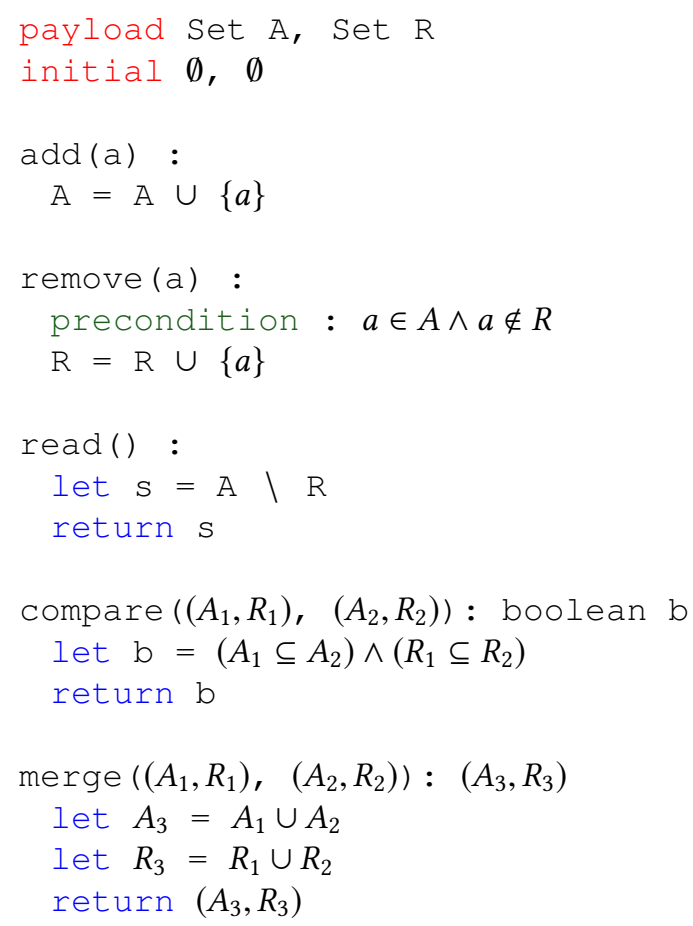

Listing 10. Pseudo-code of state-based 2P-set

\section{E.4 State-Based Two-Phase Set (2P-Set), Its Sequential Specification and Its "Local" Effectors}

Implementation: The state-based 2P-set implementation of [Shapiro et al. 2011] is given in Listing 10. It implements a set interface with operations: $\operatorname{add}(a), \operatorname{remove}(a)$ and $\operatorname{read}$. A payload $(A, R)$ contains a set $A$ to record the inserted values, and a set $R$ to store the removed values and is used as tombstone. Adding or removing a value twice has no effect, nor does adding an element that has already been removed. Therefore, we assume that the users guarantee that, in each execution, a value will not be added twice.

A value $b$ is "in the set", if $b \in A \backslash R$. add $(a)$ puts $a$ into set $A$. remove $(a)$ puts $a$ into set $R$. read returns the set content.

Sequential Specification: 2P-set uses the sequential specification $\operatorname{Spec}(\mathrm{Set})$.

The "Local" Effector, Arguments, and Predicate $P 2$ : The state-based LWW-element-set is idempotent effectors. Given operation $\ell=\operatorname{add}(a)$, then, $\arg (\ell)=(a d d, a)$; given operation $\ell=\operatorname{remove}(a)$, then, $\arg (\ell)=(\mathrm{rem}, a)$. The apply method is defined as follows: Given $\sigma=(A, R)$ and $\arg (\ell)=$ $(a d d, a), \operatorname{apply}(\sigma, \arg (\ell))=(A \cup\{a\}, R)$. Given $\sigma=(A, R)$ and $\arg (\ell)=($ rem, $a), \operatorname{apply}(\sigma, \arg (\ell))=$ $(A, R \cup\{a\})$. Predicate $P 2$ is defined as follows: Given $\sigma=(A, R)$ and $\arg (\ell)=(a d d, a), P 2(\sigma, \arg (\ell))$ holds, if $a \notin A$; Given $\sigma=(A, R)$ and $\arg (\ell)=($ rem, $a), P 2(\sigma, \arg (\ell))$ holds, if $a \notin R$.

\section{F STRUCTURE OF OUR PROOF CODE FILES}

Proof of each operation-based CRDT $c$ is divided into two files. shortname(c)_Ref_Boogie.bpl proves that all the effectors and generators of $c$ preserves the refinement relation. Whereas, shor tname( c)_Com_Boogie.bpl shows that the effectors of $c$ commute with respect to each other. 
For the state based CRDTs, proof is divided into two files as well. shortname(c)_Prop_Boogie.bpl proves that $c$ satisfies properties Prop $_{1}$ (rep., Prop ${ }_{1}^{\prime}$ ), Prop $_{2}$ (resp., Prop ${ }_{2}^{\prime}$ ), Prop $_{3}$ (resp., Prop ${ }_{3}^{\prime}$ ), Prop Pr $_{4}$ and $\operatorname{Prop}_{5}$ (or to $\operatorname{Prop}_{6}$ if $c$ is of second case and satisfies $C 1$ ). On the other hand shortname $(c)$ Ref $_{-}$ Boogie.bpl again shows that all the effectors and generators of $c$ preserves the refinement relation. shortname $(c)$ is a function that shortens the name of the CRDTs for using in the files. It is defined as:

- Two-Phase Set $\Rightarrow$ 2PSet,

- Counter $\Rightarrow \mathrm{Ctr}$,

- Last-Writer-Win Register $\Rightarrow$ LWWReg

- Last-Writer-Win-Element-Set $\Rightarrow$ LWWSet

- Multi-Value Register $\Rightarrow$ MVReg

- OR Set $\Rightarrow$ ORSet

- PN-Counter $\Rightarrow$ PNCounter

- RGA $\Rightarrow$ RGA

- Wooki $\Rightarrow$ Wookie 